\title{
Correlation functions of the integrable isotropic spin-1 chain: algebraic expressions for arbitrary temperature
}

\author{
Andreas Klümper*, Dominic Nawrath ${ }^{\dagger}$ \\ Fachbereich C - Physik, Bergische Universität Wuppertal, \\ 42097 Wuppertal, Germany \\ Junji Suzuki \\ Department of Physics, Faculty of Science, Shizuoka University, \\ Ohya 836 , Suruga, Shizuoka, Japan
}

\begin{abstract}
We derive algebraic formulas for the density matrices of finite segments of the integrable $s u(2)$ isotropic spin-1 chain in the thermodynamic limit. We give explicit results for the 2 and 3 site cases for arbitrary temperature $T$ and zero field. In the zero temperature limit the correlation functions are given in elementary form in terms of Riemann's zeta function at even integer arguments.
\end{abstract}

PACS: 05.30.-d, 75.10.Pq

*e-mail: kluemper@uni-wuppertal.de

†e-mail: dnawrath@uni-wuppertal.de

‡e-mail: sjsuzuk@ipc.shizuoka.ac.jp 


\section{Introduction}

In recent years an abundance of knowledge on the correlation functions of integrable lattice systems has been obtained. Most of the development took place for the static correlations of systems with spin-1/2 representations of the algebra $s u(2)$ including various anisotropic and inhomogeneous deformations.

The developments started with the discovery of multiple integral representations for the density matrix of a finite chain segment $[15,19,20,27]$ and the observation [8] of the factorization of these integrals into sums over products of single integrals. With $[4,10]$ it became apparent that existence and factorization of multiple integral formulas are not tied to special properties of ground-states of infinite systems that often have higher symmetries. This holds even for finite temperature in the thermodynamic limit as well as for the ground states of finite chains motivating the research $[3,6,7,21]$. Multiple integral formulations led to ab initio calculations of the asymptotics of ground state correlators of the XXZ chain [26]. Results for the closely related scalar Bose gas and for the Sine-Gordon model were obtained in [22, 25, 32, 33].

Meanwhile the 'hidden Grassmann structure' was identified [6,7] and made it possible to prove the complete factorization of general static correlation functions in terms of elementary nearest-neighbour functions under very general conditions [3,21].

In our view the most interesting question is if and how the investigations can be extended to models based on representations of higher rank algebras or to those based on higher level representations of $s u(2)$. We believe that the latter goal is in closer reach, but still very challenging. In particular, on the basis of [21] we expect algebraically transparent and physically relevant expressions for higher-spin generalizations constructed by means of the fusion procedure [30,31].

Some older results of multiple integral type exist for the ground state and were derived in the $q$-vertex operator approach in $[9,18]$ and in the combinatorial application of the algebraic Bethe ansatz in [24] for the isotropic case which was generalized to the zero field XXZ-case in [11]. The generalization of [24] to finite temperature and field was undertaken in [16]. Unfortunately, the resulting formulae are rather intricate for applications. In the present publication we undertake an effort to directly construct algebraic expressions for the finite temperature density matrices containing all information on correlation functions restricted to finite segments of infinitely long chains. Finite temperatures are treated by suitable 'lattice path integral' formulations, i.e. mapping the $1 \mathrm{~d}$ quantum chain to a suitable $2 \mathrm{~d}$ classical system. Physical quantities are obtained by a combination of algebraic techniques ( $T$ - and $Y$-systems of transfer matrices) and analytical means (functional equations).

A basic notion in the 'lattice path integral' approach to finite temperatures is the quantum transfer matrix (QTM) [35] acting in chain direction and being the 'quantum analogue' of quantum chains to the 'classical transfer matrix' of the classical Ising spin chain. The QTM satisfies the so-called $Y$-system from which by algebraic and analytic means the well-known thermodynamical Bethe ansatz (TBA) equations can be derived $[23,28]$. Another way of analysis makes use of a finite number of auxiliary functions which satisfy a closed set of functional equations. This approach was developed for the spin-1/2 case of XXX, XXZ and XYZ [29] and is probably the least canonical 
formulation, but highly efficient especially in applications. The generalization to the higher spin case was not clear before the work [34]. As we shall see below the auxiliary functions introduced in [34] are indeed most useful also for the explicit computation of correlation function of higher spin systems.

In this work we derive algebraic expressions for the correlation functions of the integrable $s u(2)$ invariant spin-1 chain at arbitrary temperature, but zero field. We will be explicit for the 2-site case and give results for 3 sites. As the reader will see, the generalization to more sites and higher spin systems is viable.

The paper is organized as follows. In section 2 we recall the construction of the Hamiltonian, the fusion procedure and fundamentals of thermodynamics. In section 3 we introduce auxiliary density matrices and computational 'tricks', especially functional equations and the algebraic Bethe ansatz for a QTM with modifications yielding a 'generating function' for the fundamental nearest-neighbour correlator in terms of which any other static correlation function factorizes. The fundamental nearest-neighbour correlator is characterized in section 4 in terms of useful integral equations. Explicit results in the low- and high-temperature limits are given. In section 5 we present concrete results for 2 sites. Section 6 is devoted to the complete computation of the 3 site density matrix. A conclusion is given which is followed by appendices containing details of our construction.

\section{Fundamental thermodynamical and integrable structures}

\subsection{Hamiltonian}

The Hamiltonian of the integrable isotropic spin-1 chain on a lattice of $L$ sites is

$$
H=\frac{J}{4} \sum_{n=1}^{L}\left[\vec{S}_{n-1} \cdot \vec{S}_{n}-\left(\vec{S}_{n-1} \cdot \vec{S}_{n}\right)^{2}\right],
$$

with for instance periodic boundary conditions $\vec{S}_{L+1}=\vec{S}_{1}$. The spin components $S_{n}^{\alpha}$ act locally as standard spin- 1 operators, and antiferromagnetic exchange, $J>0$, is assumed throughout the paper.

The Hamiltonian (1) was first obtained in a more general anisotropic form in [39]. Shortly later it was constructed by means of the fusion procedure [30,31]. The ground state and the elementary excitations were studied in [38], and an algebraic Bethe ansatz and the thermodynamics within the TBA approach were obtained in [2].

\subsection{Integrable structure}

The model can be constructed by means of the fusion procedure [30], starting from the fundamental spin- $\frac{1}{2} R$-matrix

$$
R^{[1,1]}(\lambda)=\left(\begin{array}{cccc}
1 & & & \\
& b(\lambda) & c(\lambda) & \\
& c(\lambda) & b(\lambda) & \\
& & & 1
\end{array}\right), \quad b(\lambda)=\frac{\lambda}{\lambda+2 \mathrm{i}}, \quad c(\lambda)=\frac{2 \mathrm{i}}{\lambda+2 \mathrm{i}}
$$


which we think of as an element of $\operatorname{End}\left(\mathbb{C}^{2} \otimes \mathbb{C}^{2}\right)$ (superscripts 1 indicate the spin-1/2 aka level 1 representations). It satisfies the Yang-Baxter equation

$$
R_{12}^{[1,1]}(\lambda-\mu) R_{13}^{[1,1]}(\lambda) R_{23}^{[1,1]}(\mu)=R_{23}^{[1,1]}(\mu) R_{13}^{[1,1]}(\lambda) R_{12}^{[1,1]}(\lambda-\mu) .
$$

As usual the $R_{j k}^{[1,1]}$ in this equation act on the $j$ th and $k$ th factor of the triple tensor product $\mathbb{C}^{2} \otimes \mathbb{C}^{2} \otimes \mathbb{C}^{2}$ as $R^{[1,1]}$ and on the remaining factor trivially. $R^{[1,1]}$ is normalized in such a way that

$$
R^{[1,1]}(0)=P^{[1]},
$$

where $P^{[1]}$ is the transposition of the two factors in $\mathbb{C}^{2} \otimes \mathbb{C}^{2}$. We say that $R^{[1,1]}$ is regular. At the same time $\breve{R}^{[1,1]}=P^{[1]} R^{[1,1]}$ satisfies the unitarity condition

$$
\check{R}^{[1,1]}(\lambda) \check{R}^{[1,1]}(-\lambda)=\mathbb{1}_{4},
$$

with $\mathbb{1}_{n}$ denoting the $n \times n$ unit matrix.

A further property of $R^{[1,1]}$, which is at the heart of the fusion procedure, is its degeneracy at two special points,

$$
\lim _{\lambda \rightarrow \pm 2 \mathrm{i}} \frac{R^{[1,1]}(\lambda)}{2 b(\lambda)}=P^{ \pm}
$$

where $P^{ \pm}$are the orthogonal projectors onto the singlet and triplet subspaces $V^{(s)}, V^{(t)} \subset$ $\mathbb{C}^{2} \otimes \mathbb{C}^{2}$. Due to (3) and (6) we have the important relation

$$
P_{23}^{-} R_{13}^{[1,1]}(\lambda) R_{12}^{[1,1]}(\lambda+2 \mathrm{i}) P_{23}^{+}=0
$$

meaning that $R_{13}^{[1,1]}(\lambda) R_{12}^{[1,1]}(\lambda+2 \mathrm{i})$ leaves $\mathbb{C}^{2} \otimes V^{(t)}$ invariant.

Be $S: \mathbb{C}^{2} \otimes \mathbb{C}^{2} \rightarrow \mathbb{C}^{3}$ the projector onto the triplet space we write the fused $R$ matrices as

$$
\begin{aligned}
& R^{[1,2]}(\lambda)=S_{23} R_{13}^{[1,1]}(\lambda) R_{12}^{[1,1]}(\lambda+2 \mathrm{i}) S_{23}^{t}, \\
& R^{[2,1]}(\lambda)=S_{12} R_{13}^{[1,1]}(\lambda-2 \mathrm{i}) R_{23}^{[1,1]}(\lambda) S_{12}^{t}, \\
& R^{[2,2]}(\lambda)=S_{12} S_{34} R_{14}^{[1,1]}(\lambda-2 \mathrm{i}) R_{13}^{[1,1]}(\lambda) R_{24}^{[1,1]}(\lambda) R_{23}^{[1,1]}(\lambda+2 \mathrm{i}) S_{34}^{t} S_{12}^{t}
\end{aligned}
$$

acting on $\mathbb{C}^{2} \otimes \mathbb{C}^{3}, \mathbb{C}^{3} \otimes \mathbb{C}^{2}$, or $\mathbb{C}^{3} \otimes \mathbb{C}^{3}$, respectively. Superscrips 1 and 2 refer to level 1 and level 2 (spin-1/2 and spin-1) representations. Combining the Yang-Baxter equation (3) and equations (7) it is easy to see that

$$
R_{12}^{\left[2 s_{1}, 2 s_{2}\right]}(\lambda-\mu) R_{13}^{\left[2 s_{1}, 2 s_{3}\right]}(\lambda) R_{23}^{\left[2 s_{2}, 2 s_{3}\right]}(\mu)=R_{23}^{\left[2 s_{2}, 2 s_{3}\right]}(\mu) R_{13}^{\left[2 s_{1}, 2 s_{3}\right]}(\lambda) R_{12}^{\left[2 s_{1}, 2 s_{2}\right]}(\lambda-\mu),
$$

where $s_{j}=\frac{1}{2}, 1$ for $j=1,2,3$.

In particular, $R^{[2,2]}$ is a solution of the Yang-Baxter equation. With $P^{[2]}$ denoting the transposition on $\mathbb{C}^{3} \otimes \mathbb{C}^{3}$ and $\check{R}^{[2,2]}=P^{[2]} R^{[2,2]}$ we have the standard initial condition and unitarity in the following form

$$
\begin{aligned}
& R^{[2,2]}(0)=P^{[2]}, \\
& \check{R}^{[2,2]}(\lambda) \check{R}^{[2,2]}(-\lambda)=\mathbb{1}_{9} .
\end{aligned}
$$


It follows with (10a) that $R^{[2,2]}$ generates the Hamiltonian (1),

$$
H=\mathrm{i} J \sum_{n=1}^{L} h_{n-1, n}, \quad h_{n-1, n}=\left.\partial_{\lambda} \check{R}_{n-1, n}^{[2,2]}(\lambda)\right|_{\lambda=0} .
$$

\subsection{Physical density matrix}

In [14] we have set up a formalism which enables us to calculate thermal correlation functions in integrable models with $R$-matrices fulfilling (10a). It is based on the socalled quantum transfer matrix [35] and its associated monodromy matrix which are directly related to the statistical operator.

The Hamiltonian (1) preserves the total spin $S^{\alpha}=\sum_{j=1}^{L} S_{j}^{\alpha}$. For instance, the magnetization in $z$-direction is a thermodynamic quantity, and the statistical operator

$$
\rho_{L}(T, h)=\mathrm{e}^{-\beta H-2 h S^{z}}
$$

describes the spin chain (1) in thermal equilibrium at temperature $T(=1 / \beta)$ and magnetic field $h$.

The free energy per lattice site $f(T, h)$ determines the macroscopic thermodynamics of the model. For the general integrable spin- $S$ Heisenberg chain the free energy was calculated in [34].

The free energy is related to the partition function of the statistical operator $Z:=$ $\operatorname{tr} \rho_{L}$. The normalized statistical operator

$$
D_{L}:=\frac{1}{Z} \rho_{L},
$$

is known as the density operator of the total system.

It is convenient to define the reduced density matrix of a finite chain segment $[1, m]$ by tracing out all other degrees of freedom

$$
D_{[1, m]}:=\operatorname{tr}_{\{m+1, \ldots, L\}} D_{L} .
$$

The reduced density matrix is well-defined even in the thermodynamic limit $L \rightarrow \infty$. With $D_{[1, m]}$ we can calculate the expectation value of any local operator that acts trivially outside the finite segment $[1, m]$.

For any integrable model, whose $R$-matrix does not only satisfy the Yang-Baxter equation, but also the regularity and unitarity conditions (10), we can approximate the statistical operator $\rho_{L}(T, h)$ of the $L$-site Hamiltonian using the monodromy matrix of an appropriately defined vertex model with $L$ vertical lines $(1, \ldots, L)$ and $N$ alternating horizontal lines $(\overline{1}, \ldots, \bar{N}$ with $N$ even). This fact was exploited many times in the calculation of the bulk thermodynamic properties of integrable quantum chains, in particular, in case of the higher-spin integrable Heisenberg chains [34]. In [14] it was noticed that the same formalism is also useful for the calculation of thermal correlation functions. Following the general prescription in [14] we define

$$
\begin{aligned}
T_{j}^{[2]}(\lambda)=\mathrm{e}^{2 h S_{j}^{z} / T} R_{j, \bar{N}}^{[2,2]}(\lambda+\mathrm{i} u) R_{\overline{N-1}, j}^{[2,2] t_{1}}(\mathrm{i} u-\lambda) & \\
& \ldots R_{j, \overline{2}}^{[2,2]}(\lambda+\mathrm{i} u) R_{\overline{1}, j}^{[2,2] t_{1}}(\mathrm{i} u-\lambda),
\end{aligned}
$$


where $u:=-J \beta / N$ and $t_{1}$ indicates transposition with respect to the first space in a tensor product. This monodromy matrix is constructed in such a way that (see [14])

$$
\operatorname{tr}_{\overline{1} \ldots \bar{N}}\left\{T_{1}^{[2]}(0) \ldots T_{L}^{[2]}(0)\right\}=\left[1-\frac{2}{N T} \sum_{n=1}^{L}\left(J h_{n-1, n}-2 h S_{n}^{z}\right)+\mathcal{O}\left(\frac{1}{N^{2}}\right)\right]^{\frac{N}{2}} .
$$

Hence, the statistical operator $\rho_{L}$ can be approximated by $\rho_{N, L}(T, h):=$ $\operatorname{tr}_{\overline{1}} \ldots \bar{N}\left\{T_{1}^{[2]}(0) \ldots T_{L}^{[2]}(0)\right\}$. In the so-called Trotter limit of $N \rightarrow \infty$ we have $\rho_{N, L} \rightarrow \rho_{L}$.

The transfer matrix

$$
t^{[2]}(\lambda)=\operatorname{tr}_{j} T_{j}^{[2]}(\lambda)
$$

is commonly called the quantum transfer matrix. We shall recall below how it can be diagonalized by means of the algebraic Bethe ansatz [34]. Quite generally it has the remarkable property that the eigenvalue $\Lambda^{[2]}(0)$ of largest modulus of $t^{[2]}(0)$ (we call it the dominant eigenvalue) is real and non-degenerate and is separated by the rest of the spectrum by a gap [35-37]. It can further be shown that

$$
f(T, h)=-T \lim _{N \rightarrow \infty} \ln \Lambda^{[2]}(0) .
$$

Thus, the dominant eigenvalue alone determines the bulk thermodynamic properties of the spin chain.

Owing to the fact that $R^{[2,2]}$ satisfies the Yang-Baxter equation the transfer matrices $t^{[2]}(\lambda)$ form a commuting family,

$$
\left[t^{[2]}(\lambda), t^{[2]}(\mu)\right]=0 .
$$

It follows that the eigenvectors of $t^{[2]}(\lambda)$ do not depend on $\lambda$. Let $|\Psi\rangle$ denote a normalized eigenvector belonging to the dominant eigenvalue $\Lambda^{[2]}(0)$. We shall call it the dominant eigenvector. It is unique up to normalization and is an eigenvector of $t^{[2]}(\lambda)$ with eigenvalue $\Lambda^{[2]}(\lambda)=\left\langle\Psi\left|t^{[2]}(\lambda)\right| \Psi\right\rangle$. In [14] it was pointed out that such an eigenvector determines all static correlation functions at temperature $T$ and magnetic field $h$. In particular, it determines the density matrix (14) of any finite segment $[1, m]$,

$$
D_{[1, m]}(T, h)=\lim _{N \rightarrow \infty} \frac{\left\langle\Psi\left|T^{[2]}(0) \otimes \cdots \otimes T^{[2]}(0)\right| \Psi\right\rangle}{\left(\Lambda^{[2]}(0)\right)^{m}} .
$$

For technical reasons it is better to consider a slightly more general expression than the one under the limit, by allowing for mutually distinct spectral parameters $\xi_{j}$, $j=1, \ldots, m$, instead of zero. Setting $\xi=\left(\xi_{1}, \ldots, \xi_{m}\right)$ we define

$$
D^{[2]}\left(\xi_{1}, \ldots, \xi_{m}\right)=\frac{\left\langle\Psi\left|T^{[2]}\left(\xi_{1}\right) \otimes \cdots \otimes T^{[2]}\left(\xi_{m}\right)\right| \Psi\right\rangle}{\Lambda^{[2]}\left(\xi_{1}\right) \ldots \Lambda^{[2]}\left(\xi_{m}\right)},
$$

the inhomogeneous density matrix at finite Trotter number. Then

$$
D_{[1, m]}(T, h)=\lim _{N \rightarrow \infty} \lim _{\xi_{1}, \ldots, \xi_{m} \rightarrow 0} D^{[2]}(\xi) .
$$




\section{Modified lattice model and auxiliary density matrices}

The physically interesting correlation functions are spin-1 correlators, however, the most fundamental objects are spin-1/2 correlators resp. spin-1/2 density matrices

$$
D^{[1]}\left(\widetilde{\xi}_{1}, \ldots, \widetilde{\xi}_{n}\right)=\frac{\left\langle\Psi\left|T^{[1]}\left(\widetilde{\xi}_{1}\right) \otimes \cdots \otimes T^{[1]}\left(\widetilde{\xi}_{n}\right)\right| \Psi\right\rangle}{\Lambda^{[1]}\left(\widetilde{\xi}_{1}\right) \ldots \Lambda^{[1]}\left(\widetilde{\xi}_{n}\right)} .
$$

Both types of density matrices are properly normalized, i.e. the total traces evaluate to 1 , because of the normalization terms in the denominators. Taking traces of $D^{[j]}$ turns the monodromy matrices in the numerators $T^{[j]}(\xi)$ into transfer matrices $t^{[j]}(\xi)$ which evaluate to the eigenvalue $\Lambda^{[j]}(\xi)$ when acting on the eigenstate $|\Psi\rangle$.

The density matrix $D^{[2]}\left(\xi_{1}, \ldots, \xi_{m}\right)$ is obtained from $D^{[1]}\left(\widetilde{\xi}_{1}, \ldots, \widetilde{\xi}_{n}\right)$ by fusion in the case $n=2 m$ and symmetrizations over neighbour pairs of quantum spaces carrying spectral parameters $\widetilde{\xi}_{2 k-1}=\xi_{k}-\mathrm{i}, \widetilde{\xi}_{2 k}=\xi_{k}+\mathrm{i}$. This procedure yields the unnormalized $D^{[2]}\left(\xi_{1}, \ldots, \xi_{m}\right)$. The normalization factor is given by the ratio of denominators occuring in (23), (21)

$$
\prod_{k=1}^{m} \frac{\Lambda^{[1]}\left(\xi_{k}-\mathrm{i}\right) \Lambda^{[1]}\left(\xi_{k}+\mathrm{i}\right)}{\Lambda^{[2]}\left(\xi_{k}\right)}
$$

In this paper we follow the strategy of (discrete) functional equations for finite temperature [1] to calculate the spin-1 correlators. This approach is actually formulated and viable for finite Trotter number $N$. It yields $N$ many equations for the density matrix $D^{[2]}\left(\xi_{1}, \ldots, \xi_{m}\right)$ as function of one of its spectral parameter arguments, let us say the last one $\xi_{m}$. Together with the asymptotic condition $\lim _{\xi_{m} \rightarrow \infty} D^{[2]}\left(\xi_{1}, \ldots, \xi_{m-1}, \xi_{m}\right)=$ $D^{[2]}\left(\xi_{1}, \ldots, \xi_{m-1}\right) \otimes \mathbb{1}$ (for zero field) it fixes the dependence of $D^{[2]}\left(\xi_{1}, \ldots, \xi_{m}\right)$ on $\xi_{m}$ if the data $D^{[2]}\left(\xi_{1}, \ldots, \xi_{m-1}\right)$ are given and suitable analyticity conditions hold. The analyticity conditions in the spin-1/2 case [1] are simple: The entries of the density matrix for a system with Trotter number $N$ are multivariate polynomials of degree $N$ divided by the known multivariate polynomial $\Lambda\left(\xi_{1}\right) \ldots \Lambda\left(\xi_{m}\right)$. Hence, the $N+1$ many equations (linear independence was proven in [1]) fix the $N+1$ many coefficients.

For our case at hand two different approaches are in principle conceivable: (i) the spin-1 object $D^{[2]}\left(\xi_{1}, \ldots, \xi_{m}\right)$ is tackled directly, or (ii) the spin- $1 / 2$ object $D^{[1]}\left(\widetilde{\xi}_{1}, \ldots, \widetilde{\xi}_{n}\right)$ is treated first and then fusion is applied. Unfortunately, both approaches present problems: (i) the object $D^{[2]}\left(\xi_{1}, \ldots, \xi_{m}\right)$ consists of multivariate polynomials of degree $2 N\left(\right.$ !); (ii) for $D^{[1]}\left(\widetilde{\xi}_{1}, \ldots, \widetilde{\xi}_{n}\right)$ which consists of multivariate polynomials of degree $N$ the functional equations can not be derived. In both cases though, the problems can be overcome.

\subsection{Functional properties of $D^{[2]}\left(\xi_{1}, \ldots, \xi_{m}\right)$}

In this paragraph we review the basic constructions for setting up (discrete) functional equations for reduced density matrices of integrable vertex models on semi-infinite $(\infty \times N)$ square lattices. It is possible, and for later purposes convenient, to start with a system with $N$ many different, but fixed spectral parameters $u_{j}$ on the $N$ many horizontal lines. The (unnormalized) density matrix on a finite sequence of sites has 
matrix elements that are partition functions of 'sliced lattices'. For notational simplicity we write $D\left(\xi_{1}, \ldots, \xi_{m}\right)$ for $D^{[2]}\left(\xi_{1}, \ldots, \xi_{m}\right)$ wherever possible. The local $R$-matrix enjoys

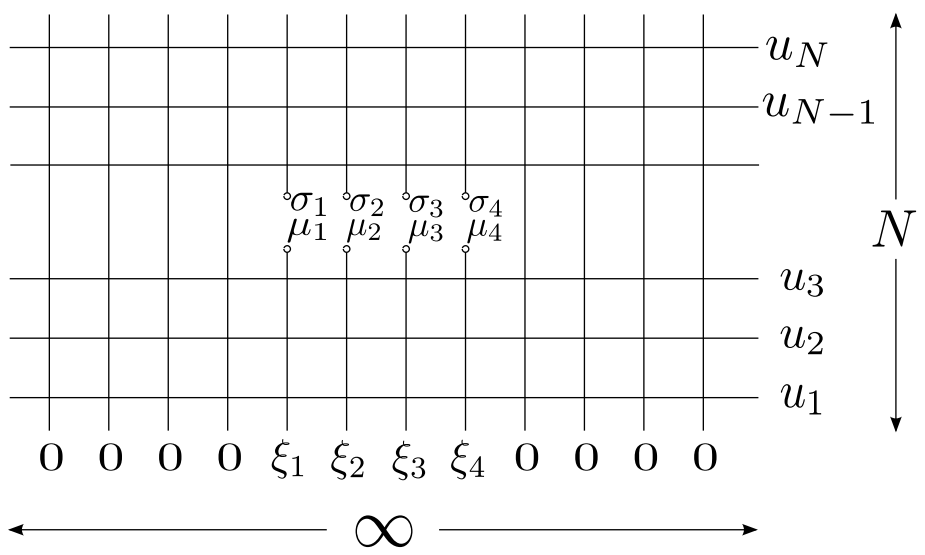

Figure 1: Graphical illustration of the matrix element $D_{\sigma_{1}, \ldots, \sigma_{m}}^{\mu_{1}, \ldots, \mu_{m}}\left(\xi_{1}, \ldots, \xi_{m}\right)$ for $m=$ 4. The lattice is semi-infinite with $N$ many infinitely long horizontal lines carrying independent spectral parameters $u_{1}, \ldots, u_{N}$.

standard initial condition and crossing symmetry which allows to perform 'lattice surgery' as described in [1]. If one of the spectral parameters $\xi_{1}, \ldots, \xi_{m}$, let us say the last one $\xi_{m}$, on the vertical lines with cut happens to be identical to any of the spectral parameters on the horizontal lines, the matrix $D\left(\xi_{1}, \ldots, \xi_{m}\right)$ maps to $D\left(\xi_{1}, \ldots, \xi_{m}-2 \mathrm{i}\right)$ upon a linear map $A$ acting on the ( $2^{2 m}$-dimensional) space of reduced density matrices. This yields the (discrete) qKZ-type functional equation
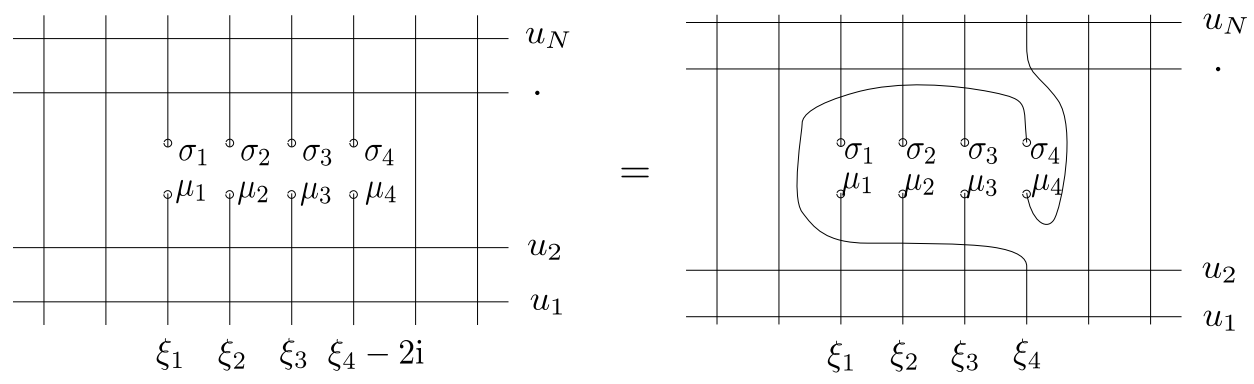

Figure 2: Graphical illustration of the functional equation (25).

$$
D\left(\xi_{1}, \ldots, \xi_{m}-2 \mathrm{i}\right)=A\left(\xi_{1}, \ldots, \xi_{m}\right)\left(\xi_{1}, \ldots, \xi_{m}\right) .
$$

The map $A$ depends on the spectral parameters $\xi_{1}, \ldots, \xi_{m}$ and is given by a product of $2 m-2$ many $R$-matrices.

In addition we have the asymptotic condition

$$
\lim _{\xi_{m} \rightarrow \infty} D\left(\xi_{1}, \ldots, \xi_{m-1}, \xi_{m}\right)=D\left(\xi_{1}, \ldots, \xi_{m-1}\right) \otimes \mathbb{1}
$$


The under-determinacy of the functional equations for the density matrix as function of a single variable is simply resolved by considering the full dependence on all arguments and invoking the intertwining (symmetry) relations

$$
\check{R}_{k, k+1}\left(\xi_{k}, \xi_{k+1}\right) D\left(\xi_{1}, \ldots, \xi_{k}, \xi_{k+1}, \ldots, \xi_{m}\right) \check{R}_{k, k+1}^{-1}\left(\xi_{k}, \xi_{k+1}\right)=D\left(\xi_{1}, \ldots, \xi_{k+1}, \xi_{k}, \ldots, \xi_{m}\right) .
$$

Let us be explicit for the 2-site case which is non-trivial and will be analyzed in detail in the next sections.

$$
\check{R}_{1,2}\left(\xi_{1}, \xi_{2}\right) D^{[2]}\left(\xi_{1}, \xi_{2}\right) \check{R}_{1,2}^{-1}\left(\xi_{1}, \xi_{2}\right)=D^{[2]}\left(\xi_{2}, \xi_{1}\right) .
$$

Here we explicitly remind of the spin-1 (level 2) case by the superscript 2. Due to $s u(2)$ invariance some simplification occurs which is not essential for the following reasoning, but leads to some notational simplification. The 2-site density matrix as well as the intertwiner $\check{R}_{1,2}$ are superpositions of projectors onto singlet, triplet and quintuplet spaces. All of these operators commute. Hence, $\check{R}_{1,2}$ and $\check{R}_{1,2}^{-1}$ on the left hand side of (28) drop out and we obtain $D^{[2]}\left(\xi_{1}, \xi_{2}\right)=D^{[2]}\left(\xi_{2}, \xi_{1}\right)$. Each of the entries of this density matrix is a symmetric polynomial of two arguments of degree $2 N$ (divided by the product of eigenvalues $\left.\Lambda^{[2]}\left(\xi_{1}\right) \Lambda^{[2]}\left(\xi_{2}\right)\right)$. Each of these polynomials has $(2 N+1)^{2}$ coeffcients. Due to symmetry, only $(2 N+1)(N+1)$ coefficients are independent. There are equations for $D^{[2]}\left(\xi_{1}, \xi_{2}\right)$ for arbitrary first argument $\xi_{1}$ and for $N+1$ many discrete values of the second argument $\xi_{2}=u_{1}, \ldots, u_{n}, \infty$ which amount to $(2 N+1)(N+1)$ many equations for the coefficients. (We have checked linear independence for characteristic cases.)

The above reasoning shows that the solution to the functional equation (25), asymptotics (26) and symmetry (27) is unique for the analytic condition that each matrix element is of the type

$$
\frac{p\left(\xi_{1}, \ldots, \xi_{m}\right)}{\Lambda^{[2]}\left(\xi_{1}\right) \ldots \Lambda^{[2]}\left(\xi_{m}\right)}
$$

where $p$ is a multivariate polynomial of degree $2 N$. Finding the solution is a different story and is based on a suitable ansatz. The result will be given in the next sections.

The above reasoning was done for pairwise different spectral parameters on the horizontal lines of the lattice. This allowed for a simple counting of independent equations. In the case of degenerate values, the equations can be formulated for the functions and for certain derivatives yielding the same counting. In the following we are dealing with systems with $N / 2$ many spectral parameters $i u$ and $N / 2$ many spectral parameters $2 \mathrm{i}-\mathrm{i} u$. Clearly, the choice of these parameters can be relaxed to have pairwise different numbers that still approximate the statistical operator.

\subsection{Functional properties of $D^{[1]}\left(\xi_{1}, \ldots, \xi_{n}\right)$}

From a fundamental point of view it would be highly desirable to tackle the density matrix $D^{[1]}\left(\xi_{1}, \ldots, \xi_{n}\right)$ (vertical spin-1/2 lines embedded in spin-1 lines). There are no clear functional properties like in the $D^{[2]}$ case as here the mixed $R$-matrices do not enjoy the standard initial condition. However, $D^{[1]}\left(\xi_{1}, \ldots, \xi_{n}\right)$ may be obtained in a certain limit of a system with (clearly) $n$ many vertical spin-1/2 lines, $2 N$ many horizontal 


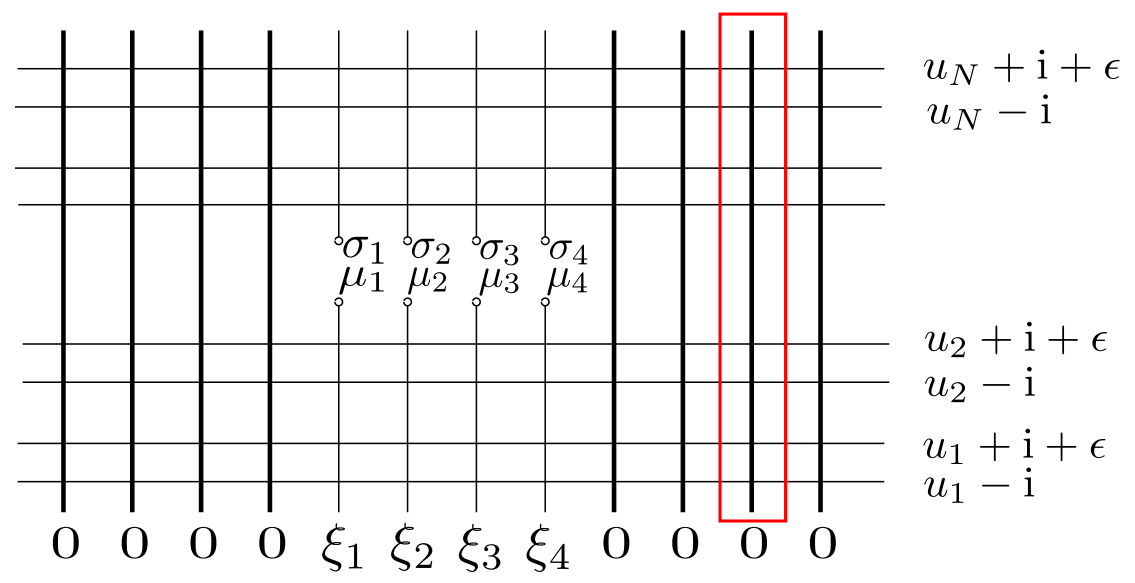

Figure 3: Graphical illustration of the auxiliary lattice. If $\varepsilon=0$ is chosen and symmetrizers at neighbouring pairs of horizontal lines with spectral parameters $u_{j} \pm \mathrm{i}$ are attached, the lattice will turn into a realization of $D^{[1]}\left(\xi_{1}, \ldots, \xi_{n}\right)$. In the main text, an argument is given to as why the same result appears in a simple $\varepsilon \rightarrow 0$ limit without symmetrizers. This argument is based on the infinitely many column-to-column transfer matrices with spin-1 auxiliary space, cf. the boxed object in the figure.

spin- $1 / 2$ lines and infinitely many vertical spin- 1 lines, see Fig. 3 for the case $n=4$. The naive idea is to use a direct fusion construction based on a lattice like the one shown in Fig. 3 with $\varepsilon=0$ and symmetrizers applied to the horizontal spaces. Next one would try to write down $2 N$ many functional equations of the type (25) as now the $R$-matrices of the vertical spin-1/2 lines and the $2 N$ many horizontal spin- $1 / 2$ lines enjoy the standard initial condition and crossing symmetry. Unfortunately, the spectral parameters on the horizontal lines are not independent. Only $N$ many meaningful equations can be derived, the other $N$ many attempts result in uncontrolled $0 / 0$ expressions.

The system shown in Fig. 3 is well-defined and has a regular $\varepsilon \rightarrow 0$ limit: the column-to-column transfer matrices with auxiliary spin-1 space take their leading state close to the space of states that are symmetric in neighbouring pairs. In the limit $\varepsilon \rightarrow 0$ the leading state converges to a state in the pure spin-1 subspace $\left(\mathbb{C}^{3}\right)^{\otimes N}$ of the total space $\left(\mathbb{C}^{2}\right)^{\otimes 2 N}$. This is clear from the root pattern of the Bethe ansatz solution to the system Fig. 3.

The benefit of dealing with the ' $\varepsilon$-regularized' system is the possibility to now formulate $2 N$ many functional equations. These equations are valid for any small, but finite $\varepsilon$ for the semi-infinite system. This is the program of the pure spin- $1 / 2$ case that leads to algebraic expressions of the density matrix in terms of nearest-neighbour correlators [1,21]. The algebraic structure is completely independent of $\varepsilon$ ! Just the nearest-neighbour correlators depend on $\varepsilon$, but have a regular limit. We therefore see that the factorized expressions also hold for the object $D^{[1]}\left(\xi_{1}, \ldots, \xi_{n}\right)$ which is of interest to us. Now the computational strategy is to write down the entries of $D^{[1]}\left(\widetilde{\xi}_{1}, \ldots, \widetilde{\xi}_{n}\right)$ for $n=2 m$ in terms of the fundamental nearest neighbour function $\Omega\left(\xi_{1}, \xi_{2}\right)$ that is 
calculated in the next section. Then the symmetrization in the quantum spaces (vertical lines) is to be performed for $\widetilde{\xi}_{2 k-1}=\xi_{k}-\mathrm{i}, \widetilde{\xi}_{2 k}=\xi_{k}+\mathrm{i}(k=1, \ldots, m)$.

\subsection{Bethe Ansatz solution}

For the calculation of the free energy (18) and the inhomogeneous density matrix (21) we need to know in first place the dominant eigenvector $|\Psi\rangle$ and the corresponding transfer matrix eigenvalue $\Lambda^{[2]}(\lambda)$. They can be obtained by means of the standard algebraic Bethe ansatz for the spin- $\frac{1}{2}$ generalized model (see e.g. chapter 12.1.6 of [12]), since, by the general reasoning of the fusion procedure [31], the quantum transfer matrix $t^{[2]}(\lambda)$ can be expressed in terms of a transfer matrix with spin- $\frac{1}{2}$ auxiliary space.

For our purposes we consider a staggered monodromy matrix with spin- $\frac{1}{2}$ auxiliary space and $N$ many spin- 1 quantum spaces and two spin-1/2 spaces by

$$
\begin{aligned}
T_{a}^{[1]}(\lambda+\mathrm{i})=\mathrm{e}^{\beta h \sigma_{a}^{z}} & R_{a, N+2}^{[1,1]}(\lambda-\mu+\mathrm{i}) R_{N+1, a}^{[1,1] t_{1}}(\mu+\delta-\lambda-\mathrm{i}) . \\
& \cdot R_{a, N}^{[1,2]}(\lambda+\mathrm{i} u) R_{N-1, a}^{[2,1] t_{1}}(\mathrm{i} u-\lambda) \ldots R_{a, 2}^{[1,2]}(\lambda+\mathrm{i} u) R_{1, a}^{[2,1] t_{1}}(\mathrm{i} u-\lambda) .
\end{aligned}
$$

Then, interpreting this monodromy matrix as a $2 \times 2$ matrix in the auxiliary space $a$, we define the transfer matrix and the quantum determinant (spin-0 fusion) as

$$
t^{[1]}(\lambda)=\operatorname{tr} T^{[1]}(\lambda), \quad \operatorname{det}_{\mathrm{q}} T^{[1]}(\lambda)=U\left(T^{[1]}(\lambda-\mathrm{i}) \otimes T^{[1]}(\lambda+\mathrm{i})\right) U^{t},
$$

where $U$ is the projector onto the antisymmetric state in the tensor product of the two auxiliary spaces. It follows from (8) that

$$
T^{[2]}(\lambda)=S\left(T^{[1]}(\lambda-\mathrm{i}) \otimes T^{[1]}(\lambda+\mathrm{i})\right) S^{t},
$$

where $S$ is the projector onto symmetric states in the tensor product of the two auxiliary spaces. Taking the trace and using (31) we conclude that

$$
t^{[2]}(\lambda)=t^{[1]}(\lambda-\mathrm{i}) t^{[1]}(\lambda+\mathrm{i})-\operatorname{det}_{\mathrm{q}} T^{[1]}(\lambda),
$$

Hence, since $\operatorname{det}_{\mathrm{q}} T^{[1]}(\lambda)$ commutes with $T^{[1]}(\lambda)$ [31], every eigenstate of $t^{[1]}(\lambda)$ is an eigenstate of $t^{[2]}(\lambda)$ as well.

The algebraic Bethe ansatz is based on the Yang-Baxter algebra relations

$$
\check{R}^{[1,1]}(\lambda-\mu)\left(T^{[1]}(\lambda) \otimes T^{[1]}(\mu)\right)=\left(T^{[1]}(\mu) \otimes T^{[1]}(\lambda)\right) \check{R}^{[1,1]}(\lambda-\mu)
$$

which follow from (9) and (30). Representing $T^{[1]}(\lambda)$ by the $2 \times 2$ matrix

$$
T^{[1]}(\lambda)=\left(\begin{array}{ll}
A(\lambda) & B(\lambda) \\
C(\lambda) & D(\lambda)
\end{array}\right),
$$

the quantum determinant is

$$
\operatorname{det}_{\mathrm{q}} T^{[1]}(\lambda)=D(\lambda-\mathrm{i}) A(\lambda+\mathrm{i})-B(\lambda-\mathrm{i}) C(\lambda+\mathrm{i}) .
$$


Defining the pseudo vacuum

$$
|0\rangle=\left[\left(\begin{array}{l}
0 \\
0 \\
1
\end{array}\right) \otimes\left(\begin{array}{l}
1 \\
0 \\
0
\end{array}\right)\right]^{\otimes \frac{N}{2}} \otimes\left(\begin{array}{l}
0 \\
1
\end{array}\right) \otimes\left(\begin{array}{l}
1 \\
0
\end{array}\right)
$$

we deduce from (30) that

$$
C(\lambda)|0\rangle=0, \quad A(\lambda)|0\rangle=a(\lambda)|0\rangle, \quad D(\lambda)|0\rangle=d(\lambda)|0\rangle,
$$

where the pseudo vacuum eigenvalues $a(\lambda)$ and $d(\lambda)$ are explicit complex valued functions. Using the notation

$$
\phi_{ \pm}(\lambda)=(\lambda \pm \mathrm{i} u)^{N / 2}, \quad u=-\frac{J}{N T},
$$

which proved to be useful in [34], and

$$
\varphi_{+}(\lambda):=\lambda-\mu, \quad \varphi_{-}(\lambda):=\lambda-\mu-\delta,
$$

which arise from the additional horizontal spin-1/2 lines carrying the spectral parameters $\mu-\mathrm{i}$ and $\mu-\mathrm{i}+\delta$ (at the vertex with transposition), we can express the vacuum eigenvalues as

$$
a(\lambda)=\mathrm{e}^{h / T} \frac{\phi_{-}(\lambda+\mathrm{i})}{\phi_{-}(\lambda-3 \mathrm{i})} \frac{\varphi_{-}(\lambda)}{\varphi_{-}(\lambda-2 \mathrm{i})}, \quad d(\lambda)=\mathrm{e}^{-h / T} \frac{\phi_{+}(\lambda-\mathrm{i})}{\phi_{+}(\lambda+3 \mathrm{i})} \frac{\varphi_{+}(\lambda)}{\varphi_{+}(\lambda+2 \mathrm{i})} .
$$

Given the Yang-Baxter algebra (34) and the pseudo vacuum eigenvalues (41) the eigenvectors and eigenvalues of $t^{[1]}(\lambda)$ can be obtained from general considerations (see e.g. chapter 12.1 .6 of [12]). The dominant eigenstate $|\Psi\rangle$ of $t^{[2]}(\lambda)$, in particular, can be represented as

$$
|\Psi\rangle=B\left(\lambda_{1}\right) \ldots B\left(\lambda_{N+1}\right)|0\rangle
$$

where the set of so-called Bethe roots $\left\{\lambda_{j}\right\}_{j=1}^{N+1}$ is a specific solution of the Bethe ansatz equations

$$
\frac{a\left(\lambda_{j}\right)}{d\left(\lambda_{j}\right)}=\prod_{\substack{k=1 \\ k \neq j}}^{N+1} \frac{\lambda_{j}-\lambda_{k}+2 \mathrm{i}}{\lambda_{j}-\lambda_{k}-2 \mathrm{i}}, \quad j=1, \ldots, N+1 .
$$

For the given set of Bethe roots $\left\{\lambda_{j}\right\}_{j=1}^{N+1}$ we define the $Q$-function

$$
q(\lambda)=\prod_{j=1}^{N+1}\left(\lambda-\lambda_{j}\right)
$$

Then the eigenvalue of $t^{[1]}(\lambda)$ corresponding to $|\Psi\rangle$ is

$$
\Lambda^{[1]}(\lambda)=a(\lambda) \frac{q(\lambda-2 \mathrm{i})}{q(\lambda)}+d(\lambda) \frac{q(\lambda+2 \mathrm{i})}{q(\lambda)} .
$$

As for the eigenvalue of $t^{[2]}(\lambda)$ we conclude with (33) and equation (36) above that

$$
\Lambda^{[2]}(\lambda)=\Lambda^{[1]}(\lambda-\mathrm{i}) \Lambda^{[1]}(\lambda+\mathrm{i})-a(\lambda+\mathrm{i}) d(\lambda-\mathrm{i})
$$

This eigenvalue and the Bethe ansatz equations (43) are the main input for the calculation of the thermodynamics of the spin-1 chain. In order to perform the Trotter limit the eigenvalue must be represented by means of auxiliary functions satisfying a finite set of nonlinear integral equations. This was achieved in [34]. 


\section{Integral equations and basic functions}

In this section we consider the evaluation of the largest eigenvalue of the generalized quantum transfer matrix by means of nonlinear integral equations (NLIE). This gives us the opportunity to introduce certain auxiliary functions that are also relevant for the factorized algebraic expressions of the density matrix elements in the next section. Our starting point is the expression for the dominant eigenvalue $\Lambda^{[2]}(\lambda)$ of the quantum transfer matrix together with the Bethe ansatz solution (43)-(46). In [34] the problem was solved within the more general context of the fusion hierarchy, and NLIE for the integrable isotropic spin chains of arbitrary spin were obtained.

For the calculation of the free energy for spin 1 we will be dealing with three coupled NLIE for three functions $\mathfrak{b}, \overline{\mathfrak{b}}$ and $y$. We show the equations below in (48) and present the derivation in appendix B. (Most of the relations of this section are valid for arbitrary magnetic field $h$, however the main applications later on concern the zero field case.) For finite Trotter number $N$ the functions $\mathfrak{b}, \overline{\mathfrak{b}}$ and $y$ can be expressed in terms of the $Q$-function (44) and the functions $\phi_{ \pm}$introduced in (39) (see appendix A). This defines them as meromorphic functions on the entire complex plane, but is inappropriate for performing the Trotter limit. In the NLIE, on the other hand, the Trotter number appears only in the driving term and the Trotter limit is easily obtained. For a discussion of some of the subtleties related to the Trotter limit and the definition of useful auxiliary functions see [17].

The functions $\mathfrak{b}(\lambda), \overline{\mathfrak{b}}(\lambda)$ and $y(\lambda)$ are defined in (A.5), (A.7) and will be of particular use in the neighbourhood of the real axis. For convenience we introduce the shifted functions

$$
\mathfrak{b}_{\varepsilon}(\lambda)=\mathfrak{b}(\lambda-\mathrm{i} \varepsilon), \quad \overline{\mathfrak{b}}_{\varepsilon}(\lambda)=\overline{\mathfrak{b}}(\lambda+\mathrm{i} \varepsilon),
$$

and similar capital functions, $\mathfrak{B}_{\varepsilon}(\lambda)=1+\mathfrak{b}_{\varepsilon}(\lambda)$ etc. Then the desired NLIE read

$$
\left(\begin{array}{c}
\log y(\lambda) \\
\log \mathfrak{b}_{\varepsilon}(\lambda) \\
\log \overline{\mathfrak{b}}_{\varepsilon}(\lambda)
\end{array}\right)=\left(\begin{array}{c}
\Delta_{y}(\lambda) \\
\Delta_{b}(\lambda) \\
\Delta_{\bar{b}}(\lambda)
\end{array}\right)+\widehat{\mathcal{K}} *\left(\begin{array}{c}
\log Y(\lambda) \\
\log \mathfrak{B}_{\varepsilon}(\lambda) \\
\log \overline{\mathfrak{B}}_{\varepsilon}(\lambda)
\end{array}\right)
$$

where $(\widehat{\mathcal{K}} * g)_{i}$ denotes the matrix convolution $\sum_{j} \int_{-\infty}^{\infty} \mathrm{d} \lambda^{\prime} \widehat{\mathcal{K}}_{i, j}\left(\lambda-\lambda^{\prime}\right) g_{j}\left(\lambda^{\prime}\right)$, and

$$
\begin{aligned}
\Delta_{y}(\lambda) & =\log \left[\frac{\tanh \left(\frac{\pi}{4}(\lambda-\mu+\mathrm{i})\right)}{\tanh \left(\frac{\pi}{4}(\lambda-\mu-\delta+\mathrm{i})\right)}\right] \simeq-\mathrm{i} \frac{\frac{\pi}{2}}{\cosh \frac{\pi}{2}(\lambda-\mu)} \cdot \delta, \\
\Delta_{b}(\lambda) & =-\frac{h}{T}+d(u, \lambda-\mathrm{i} \varepsilon), \quad \Delta_{\bar{b}}(\lambda)=\frac{h}{T}+d(u, \lambda+\mathrm{i} \varepsilon), \\
d(u, \lambda) & =\frac{N}{2} \int_{-\infty}^{\infty} \mathrm{d} k \mathrm{e}^{-\mathrm{i} k \lambda} \frac{\sinh u k}{k \cosh k} \stackrel{N \rightarrow \infty}{\longrightarrow}-\frac{J}{T} \frac{\frac{\pi}{2}}{\cosh \frac{\pi}{2} \lambda} .
\end{aligned}
$$

The integration constants $( \pm h / T)$ are fixed by comparing the asymptotic values of both sides of (48) for $|\lambda| \rightarrow \infty$. The kernel matrix is given by

$$
\widehat{\mathcal{K}}(\lambda)=\left(\begin{array}{ccc}
0 & \mathcal{K}(\lambda+\mathrm{i} \varepsilon) & \mathcal{K}(\lambda-\mathrm{i} \varepsilon) \\
\mathcal{K}(\lambda-\mathrm{i} \varepsilon) & \mathcal{F}(\lambda) & -\mathcal{F}(\lambda+2 \mathrm{i}(1-\varepsilon)) \\
\mathcal{K}(\lambda+\mathrm{i} \varepsilon) & -\mathcal{F}(\lambda-2 \mathrm{i}(1-\varepsilon)) & \mathcal{F}(\lambda)
\end{array}\right),
$$


where

$$
\mathcal{K}(\lambda)=\frac{1}{4 \cosh \pi \lambda / 2}, \quad \mathcal{F}(\lambda)=\int_{-\infty}^{\infty} \frac{\mathrm{d} k}{2 \pi} \frac{e^{-|k|-\mathrm{i} k \lambda}}{2 \cosh k},
$$

and $\mathcal{F}$ can be expressed in terms of the digamma function $\Psi$, see (56).

The derivation of integral expressions for the eigenvalues is involved, we defer the details to appendix B. The results are

$$
\begin{aligned}
\log \Lambda^{[1]}(\lambda)= & -\int_{-\infty}^{\infty} d k \frac{N e^{-3|k|} \cosh ((u+1) k)}{2|k| \cosh (k)} e^{-\mathrm{i} k \lambda}-\log \left[\phi_{+}(\lambda+3 \mathrm{i}) \phi_{-}(\lambda-3 \mathrm{i})\right]+\mathrm{cst} . \\
& +\int_{-\infty}^{\infty} d \lambda^{\prime} \mathcal{K}\left(\lambda-\lambda^{\prime}\right)\left\{\log Y\left(\lambda^{\prime}\right)+\log \left[\frac{\varphi_{+}(\lambda-\mathrm{i}) \varphi_{-}(\lambda+\mathrm{i})}{\varphi_{-}(\lambda-\mathrm{i}) \varphi_{+}(\lambda+\mathrm{i})}\right]\right\} \quad \text { (52a) }
\end{aligned}
$$

respectively

$$
\log \Lambda^{[2]}(\lambda)=\log \left[\frac{\phi_{+}(\lambda-2 \mathrm{i}) \phi_{-}(\lambda+2 \mathrm{i})}{\phi_{-}(\lambda-2 \mathrm{i}) \phi_{+}(\lambda+2 \mathrm{i})} \frac{\varphi_{+}(\lambda-\mathrm{i}) \varphi_{-}(\lambda+\mathrm{i})}{\varphi_{-}(\lambda-\mathrm{i}) \varphi_{+}(\lambda+\mathrm{i})}\right]+\log y(\lambda)
$$

Next we are interested in $\frac{\partial}{\partial \delta} \log \Lambda^{[1]}$ for which we derive an integral expression in terms of functions satisfying a set of linear integral equations. The basic functions are the derivatives of the functions $\log Y, \log \mathfrak{B}_{\varepsilon}, \log \overline{\mathfrak{B}}_{\varepsilon}$ with respect to $\delta$. We have the identities

$$
\begin{aligned}
& G_{y}(\lambda, \mu):=\left.\frac{\partial}{\partial \delta} \log Y(\lambda)\right|_{\delta=0},\left.\frac{\partial}{\partial \delta} \log y(\lambda)\right|_{\delta=0}=\left.\left[1+y^{-1}(\lambda)\right]\right|_{\delta=0} G_{y}(\lambda, \mu), \\
& G_{\mathfrak{b}}(\lambda, \mu):=\left.\frac{\partial}{\partial \delta} \log \mathfrak{B}_{\varepsilon}(\lambda)\right|_{\delta=0},\left.\quad \frac{\partial}{\partial \delta} \log \mathfrak{b}_{\varepsilon}(\lambda)\right|_{\delta=0}=\left.\left[1+\mathfrak{b}_{\varepsilon}^{-1}(\lambda)\right]\right|_{\delta=0} G_{\mathfrak{b}}(\lambda, \mu), \\
& G_{\overline{\mathfrak{b}}}(\lambda, \mu):=\left.\frac{\partial}{\partial \delta} \log \overline{\mathfrak{B}}_{\varepsilon}(\lambda)\right|_{\delta=0},\left.\quad \frac{\partial}{\partial \delta} \log \overline{\mathfrak{b}}_{\varepsilon}(\lambda)\right|_{\delta=0}=\left.\left[1+\overline{\mathfrak{b}}_{\varepsilon}^{-1}(\lambda)\right]\right|_{\delta=0} G_{\overline{\mathfrak{b}}}(\lambda, \mu) .
\end{aligned}
$$

These satisfy the set of linear integral equations

$$
\left(\begin{array}{l}
{\left[1+y^{-1}(\lambda)\right] G_{y}(\lambda, \mu)} \\
{\left[1+\mathfrak{b}_{\varepsilon}^{-1}(\lambda)\right] G_{\mathfrak{b}}(\lambda, \mu)} \\
{\left[1+\overline{\mathfrak{b}}_{\varepsilon}^{-1}(\lambda)\right] G_{\overline{\mathfrak{b}}}(\lambda, \mu)}
\end{array}\right)=\left(\begin{array}{c}
-\mathrm{i} \frac{\frac{\pi}{2}}{\cosh \frac{\pi}{2}(\lambda-\mu)} \\
0 \\
0
\end{array}\right)+\widehat{\mathcal{K}} *\left(\begin{array}{c}
G_{y}(\lambda, \mu) \\
G_{\mathfrak{b}}(\lambda, \mu) \\
G_{\mathfrak{b}}(\lambda, \mu)
\end{array}\right),
$$

Next we obtain an explicit expression of the eigenvalue's derivative in terms of the $G$ functions

$$
\begin{aligned}
\left.\frac{\partial}{\partial \delta} \log \Lambda^{[1]}(\lambda)\right|_{\delta=0} & =\int_{-\infty}^{\infty} d \lambda^{\prime} \frac{1}{4 \cosh \left(\frac{\pi}{2}\left(\lambda-\lambda^{\prime}\right)\right)}\left\{G_{y}\left(\lambda^{\prime}, \mu\right)+\frac{2 \mathrm{i}}{\left(\lambda^{\prime}-\mu\right)^{2}+1}\right\} \\
& =\int_{-\infty}^{\infty} d \lambda^{\prime} \frac{1}{4 \cosh \left(\frac{\pi}{2}\left(\lambda-\lambda^{\prime}\right)\right)} G_{y}\left(\lambda^{\prime}, \mu\right)+2 \pi i \mathcal{F}(\lambda-\mu)
\end{aligned}
$$


for $|\operatorname{Im}(\lambda)|<1$. Next we note a useful identity for $\mathcal{F}$ defined in (51)

$$
\mathcal{F}(\lambda)=\frac{1}{8 \pi}\left\{\Psi\left(-\frac{i}{4} \lambda\right)+\Psi\left(\frac{i}{4} \lambda\right)-\Psi\left(\frac{1}{2}-\frac{i}{4} \lambda\right)-\Psi\left(\frac{1}{2}+\frac{i}{4} \lambda\right)\right\},
$$

where $\Psi$ is the standard digamma function

$$
\Psi(x):=\frac{\partial}{\partial x} \log (\Gamma(x)), \quad \Gamma(x):=\int_{0}^{\infty} t^{x-1} e^{-t} d t .
$$

We remind of the functional equations

$$
\Psi(1-x)=\Psi(x)+\pi \operatorname{ctg}(\pi x), x \neq \frac{1}{2},
$$

and

$$
\Psi(x+1)=\Psi(x)+\frac{1}{x} .
$$

The eigenvalue's derivative satisfies the following 2-point equation

$$
\left.\frac{\partial}{\partial \delta} \ln \Lambda^{[1]}(\lambda+\mathrm{i})\right|_{\delta=0}+\left.\frac{\partial}{\partial \delta} \ln \Lambda^{[1]}(\lambda-\mathrm{i})\right|_{\delta=0}=G_{y}(\lambda, \mu)+\frac{1}{\lambda-\mu-\mathrm{i}}-\frac{1}{\lambda-\mu+\mathrm{i}} .
$$

The right hand side does not simplify in any obvious way to elementary expressions due to the occurrence of $G_{y}(\lambda, \mu)$. In the next subsection we will derive a simple 3-point equation involving just derivatives of $\Lambda^{[1]}$.

Due to $s u(2)$ invariance the density operator $D^{[1]}(\lambda, \mu)$ for two neighbouring spin$1 / 2$ spaces with spectral parameters $\lambda, \mu$ has the following representation

$$
D^{[1]}(\lambda, \mu)=\left(\frac{1}{4}-\frac{1}{6} \omega(\lambda, \mu)\right) \mathbb{1}+\frac{1}{3} \omega(\lambda, \mu) P^{[1]}
$$

where $P^{[1]}$ is the permutation operator of neighbouring spin- $1 / 2$ objects and $\omega(\lambda, \mu)$ is some (symmetric) function. In Appendix $C$ we show that

$$
\omega(\lambda, \mu)=\frac{1}{2}-\left.\frac{(\lambda-\mu)^{2}+4}{2 \mathrm{i}} \frac{\partial}{\partial \delta} \ln \left\{\Lambda^{[1]}(\lambda ; \mu)\right\}\right|_{\delta=0} .
$$

For later purposes, it is convenient to use another closely related function

$$
\begin{aligned}
\Omega(\lambda, \mu) & :=\frac{2 \mathrm{i}}{\left.(\lambda-\mu)^{2}+4\right)} \operatorname{tr}\left\{D^{[1]}(\lambda, \mu) P^{[1]}\right\} \\
& =2 \mathrm{i} \frac{\omega(\lambda, \mu)+\frac{1}{2}}{(\lambda-\mu)^{2}+4}=-\left.\frac{\partial}{\partial \delta} \ln \left\{\Lambda^{[1]}(\lambda ; \mu)\right\}\right|_{\delta=0}+\frac{2 \mathrm{i}}{(\lambda-\mu)^{2}+4}
\end{aligned}
$$

An interesting and useful observation concerns the trace of $P^{[1]}$ over the symmetric subspace for arguments $(\lambda, \mu)$ chosen as $(\lambda-i, \lambda+i)$ which according to the fusion principles yields

$$
\frac{3}{4}+\frac{1}{2} \omega(\lambda-\mathrm{i}, \lambda+\mathrm{i})=\frac{\Lambda_{2}(\lambda)}{\Lambda_{1}(\lambda+\mathrm{i}) \Lambda_{1}(\lambda-\mathrm{i})}=\frac{1}{1+y(\lambda)^{-1}} .
$$

We have the explicit expression (62) which seems to yield $\frac{1}{2}$ for $\omega(\lambda-i, \lambda+i)$. However, $\frac{\partial}{\partial \delta} \ln \Lambda^{[1]}(\lambda ; \mu)$ develops poles for $\lambda-\mu= \pm 2 \mathrm{i}$ which can be evaluated on the basis of (55a) and (54) in full agreement with (64). 


\subsection{Functional equations for the basic functions}

From the definition (A.1a), (A.1b) of the functions $\Lambda_{1}$ and $\Lambda_{2}$ we find the relations

$$
\begin{aligned}
\Lambda_{1}(\lambda-\mathrm{i}) \Lambda_{1}(\lambda+\mathrm{i}) & =\phi(\lambda-3 \mathrm{i}) \phi(\lambda+3 \mathrm{i}) \varphi(\lambda-2 \mathrm{i}) \varphi(\lambda+2 \mathrm{i})+\Lambda_{2}(\lambda) \\
& =\phi(\lambda-3 \mathrm{i}) \phi(\lambda+3 \mathrm{i}) \varphi(\lambda-2 \mathrm{i}) \varphi(\lambda+2 \mathrm{i}) \cdot Y(\lambda) \\
\Lambda_{2}(\lambda-\mathrm{i}) \Lambda_{2}(\lambda+\mathrm{i}) & =\phi(\lambda-2 \mathrm{i}) \phi(\lambda-4 \mathrm{i}) \phi(\lambda+4 \mathrm{i}) \phi(\lambda+2 \mathrm{i}) \times \\
& \times \varphi(\lambda-\mathrm{i}) \varphi(\lambda-3 \mathrm{i}) \varphi(\lambda+3 \mathrm{i}) \varphi(\lambda+\mathrm{i})+\phi(\lambda) \tilde{\Lambda}(\lambda) .
\end{aligned}
$$

We divide the first equation by $\varphi(\lambda-2 \mathrm{i}) \varphi(\lambda+2 \mathrm{i})$ and the second equation by a product of that function with $\lambda$ replaced by $\lambda \pm \mathrm{i}$

$$
\begin{aligned}
\frac{\Lambda_{1}(\lambda-\mathrm{i}) \Lambda_{1}(\lambda+\mathrm{i})}{\varphi(\lambda-2 \mathrm{i}) \varphi(\lambda+2 \mathrm{i})} & =\phi(\lambda-3 \mathrm{i}) \phi(\lambda+3 \mathrm{i})+\frac{\Lambda_{2}(\lambda)}{\varphi(\lambda-2 \mathrm{i}) \varphi(\lambda+2 \mathrm{i})} \\
\frac{\Lambda_{2}(\lambda-\mathrm{i})}{\varphi(\lambda-3 \mathrm{i}) \varphi(\lambda+\mathrm{i})} \cdot \frac{\Lambda_{2}(\lambda+\mathrm{i})}{\varphi(\lambda-\mathrm{i}) \varphi(\lambda+3 \mathrm{i})} & =\phi(\lambda-2 \mathrm{i}) \phi(\lambda-4 \mathrm{i}) \phi(\lambda+4 \mathrm{i}) \phi(\lambda+2 \mathrm{i})+ \\
& +\frac{\phi(\lambda)}{\varphi(\lambda-\mathrm{i}) \varphi(\lambda-3 \mathrm{i}) \varphi(\lambda+3 \mathrm{i}) \varphi(\lambda+\mathrm{i})} \widetilde{\Lambda}(\lambda) .
\end{aligned}
$$

From the last equation we see that

$$
\frac{\partial}{\partial \delta} \ln \frac{\Lambda_{2}(\lambda-i)}{\varphi(\lambda-3 i) \varphi(\lambda+i)}+\frac{\partial}{\partial \delta} \ln \frac{\Lambda_{2}(\lambda+i)}{\varphi(\lambda-i) \varphi(\lambda+3 i)}=0 \quad \text { if } \phi(\lambda)=0,
$$

since for $\lambda$ 's with $\phi(\lambda)=0$ the right hand side of (68) is absolutely independent of $\delta$.

$$
\begin{aligned}
\frac{\partial}{\partial \delta} \ln \frac{\Lambda_{2}(\lambda)}{\varphi(\lambda-2 i) \varphi(\lambda+2 \mathrm{i})} & =\frac{\varphi(\lambda-2 \mathrm{i}) \varphi(\lambda+2 \mathrm{i})}{\Lambda_{2}(\lambda)} \frac{\partial}{\partial \delta} \frac{\Lambda_{2}(\lambda)}{\varphi(\lambda-2 \mathrm{i}) \varphi(\lambda+2 \mathrm{i})} \\
& =\frac{\varphi(\lambda-2 \mathrm{i}) \varphi(\lambda+2 \mathrm{i})}{\Lambda_{2}(\lambda)} \frac{\partial}{\partial \delta} \frac{\Lambda_{1}(\lambda-\mathrm{i}) \Lambda_{1}(\lambda+\mathrm{i})}{\varphi(\lambda-2 \mathrm{i}) \varphi(\lambda+2 \mathrm{i})} \\
& =\frac{\Lambda_{1}(\lambda-\mathrm{i}) \Lambda_{1}(\lambda+\mathrm{i})}{\Lambda_{2}(\lambda)} \frac{\partial}{\partial \delta} \ln \frac{\Lambda_{1}(\lambda-\mathrm{i}) \Lambda_{1}(\lambda+\mathrm{i})}{\varphi(\lambda-2 \mathrm{i}) \varphi(\lambda+2 \mathrm{i})}
\end{aligned}
$$

where for the second identity we have used (67) where the first summand on the right hand side is independent of $\delta$. Next, inside the logarithm, we replace $\Lambda_{1}$ by $\Lambda^{[1]}$ and the logarithmic derivative by the function $\Omega$ (63). We then find for the right hand side of (70)

$$
\ldots=\frac{\Lambda_{1}(\lambda-\mathrm{i}) \Lambda_{1}(\lambda+\mathrm{i})}{\Lambda_{2}(\lambda)}\left(\frac{3 \mathrm{i}}{(\lambda-\mu)^{2}+9}-\frac{\mathrm{i}}{(\lambda-\mu)^{2}+1}-\Omega(\lambda-\mathrm{i}, \mu)-\Omega(\lambda+\mathrm{i}, \mu)\right) .
$$

The ratio of $\Lambda$ functions can be simplified by use of (64). With

$$
N(\xi):=\frac{3}{4}+\frac{1}{2} \omega(\xi-\mathrm{i}, \xi+\mathrm{i}), \quad o(\lambda):=\frac{2 \mathrm{i}\left(\lambda^{2}-3\right)}{(\lambda-3 \mathrm{i})(\lambda-\mathrm{i})(\lambda+\mathrm{i})(\lambda+3 \mathrm{i})} .
$$


we obtain

$$
\frac{\partial}{\partial \delta} \ln \frac{\Lambda_{2}(\lambda)}{\varphi(\lambda-2 \mathrm{i}) \varphi(\lambda+2 \mathrm{i})}=-\frac{1}{N(\lambda)}(\Omega(\lambda-\mathrm{i}, \mu)+\Omega(\lambda+\mathrm{i}, \mu)-o(\lambda-\mu)) .
$$

Finally, the functional equation (69) takes the form

$$
\frac{\Omega(\lambda-2 \mathrm{i}, \mu)+\Omega(\lambda, \mu)-o(\lambda-\mu-\mathrm{i})}{N(\lambda-\mathrm{i})}+\frac{\Omega(\lambda, \mu)+\Omega(\lambda+2 \mathrm{i}, \mu)-o(\lambda-\mu+\mathrm{i})}{N(\lambda+\mathrm{i})}=0,
$$

for all $\lambda$ being zeros of $\phi(\lambda)$.

\subsection{Low and high temperature limits}

Low temperature limit, zero field

From (48) we read off for zero field $h=0$ the zero-temperature limit $T \rightarrow 0$ of the auxiliary functions $y, \mathfrak{b}_{\varepsilon}$ and $\overline{\mathfrak{b}}_{\varepsilon}$

$$
\left.\lim _{T \rightarrow 0} y\right|_{\delta=0}=1,\left.\lim _{T \rightarrow 0} \mathfrak{b}_{\varepsilon}\right|_{\delta=0}=0=\left.\lim _{T \rightarrow 0} \overline{\mathfrak{b}}_{\varepsilon}\right|_{\delta=0} .
$$

And from (54) we obtain in the same limit

$$
G_{y}(\lambda, \mu)=-\frac{\mathrm{i}}{2} \frac{\frac{\pi}{2}}{\cosh \frac{\pi}{2}(\lambda-\mu)}, \quad G_{\mathfrak{b}}(\lambda, \mu)=G_{\mathfrak{b}}(\lambda, \mu)=0 .
$$

Hence the zero-temperature limit of the functions $\Omega$ and $\omega(63)$ is calculated directly from (55b) yielding

$$
\begin{aligned}
& \lim _{T \rightarrow 0} \omega(\lambda, \mu)=\frac{1}{2}-\frac{1}{8}\left((\lambda-\mu)^{2}+4\right)\left(\left\{\Psi\left(-\frac{i}{4}(\lambda-\mu)\right)+\Psi\left(\frac{i}{4}(\lambda-\mu)\right)\right.\right. \\
&\left.\left.-\Psi\left(\frac{1}{2}-\frac{i}{4}(\lambda-\mu)\right)-\Psi\left(\frac{1}{2}+\frac{i}{4}(\lambda-\mu)\right)\right\}-\frac{\pi(\lambda-\mu)}{2 \sinh \left(\frac{\pi}{2}(\lambda-\mu)\right)}\right) .
\end{aligned}
$$

High temperature expansion, zero field

We expand the auxiliary functions in powers of $1 / T$

$$
\begin{aligned}
\left.y(\lambda)\right|_{\delta=0} & =y_{0}+\sum_{k=1}^{\infty}\left(\frac{1}{T}\right)^{k} y_{k}(\lambda), \\
\left.\mathfrak{b}_{\varepsilon}(\lambda)\right|_{\delta=0} & =\mathfrak{b}_{0}+\sum_{k=1}^{\infty}\left(\frac{1}{T}\right)^{k} \mathfrak{b}_{k}(\lambda), \\
\left.\overline{\mathfrak{b}}_{\varepsilon}(\lambda)\right|_{\delta=0} & =\overline{\mathfrak{b}}_{0}+\sum_{k=1}^{\infty}\left(\frac{1}{T}\right)^{k} \overline{\mathfrak{b}}_{k}(\lambda),
\end{aligned}
$$

and solve the NLIE iteratively. The $0^{\text {th }}$ order is solved by the constants $y_{0}=3, \mathfrak{b}_{0}=$ $\overline{\mathfrak{b}}_{0}=2$. The NLIE for the auxiliary functions up to $1^{\text {st }}$ order linearize in $y_{1}(\lambda), \mathfrak{b}_{1}(\lambda)$, $\overline{\mathfrak{b}}_{1}(\lambda)$ and can be solved by Fourier transformation. This can be repeated over and over 
again: the NLIE linearize in the next (unknown) order where the (known) lower order terms appear in non-linear combinations as driving terms. We restrict ourselves to the first order calculation

$$
\begin{aligned}
\left.y(\lambda)\right|_{\delta=0} & =3-\frac{128}{\left(4+\lambda^{2}\right)\left(16+\lambda^{2}\right)} \frac{J}{T}+\mathcal{O}\left(1 / T^{2}\right), \\
\left.\mathfrak{b}_{\varepsilon}(\lambda)\right|_{\delta=0} & =2-\frac{32 \mathrm{i}\left(4+\mathrm{i}(\lambda-\mathrm{i} \varepsilon)+(\lambda-\mathrm{i} \varepsilon)^{2}\right)}{\left((\lambda-\mathrm{i} \varepsilon)^{2}+1\right)\left((\lambda-\mathrm{i} \varepsilon)^{2}+9\right)(\lambda-\mathrm{i} \varepsilon+5 \mathrm{i})} \frac{J}{T}+\mathcal{O}\left(1 / T^{2}\right), \\
\left.\overline{\mathfrak{b}}_{\varepsilon}(\lambda)\right|_{\delta=0} & =2+\frac{32 \mathrm{i}\left(4-\mathrm{i}(\lambda+\mathrm{i} \varepsilon)+(\lambda+\mathrm{i} \varepsilon)^{2}\right)}{\left((\lambda+\mathrm{i} \varepsilon)^{2}+1\right)\left((\lambda+\mathrm{i} \varepsilon)^{2}+9\right)(\lambda+\mathrm{i} \varepsilon-5 \mathrm{i})} \frac{J}{T}+\mathcal{O}\left(1 / T^{2}\right) .
\end{aligned}
$$

Using this strategy also for the auxiliary functions $G_{y}, G_{\mathfrak{b}}, G_{\overline{\mathfrak{b}}}$, the high temperature expansion for the logarithmic derivative of the eigenvalue with respect to $\delta$ leads to

$$
\left.\frac{\partial}{\partial \delta} \log \Lambda^{[1]}(\lambda)\right|_{\delta=0}=\frac{\mathrm{i}}{(\lambda-\mu)^{2}+4}\left\{1+\frac{32}{\left(9+\lambda^{2}\right)\left(9+\mu^{2}\right)} \frac{J}{T}\right\}+\mathcal{O}\left(1 / T^{2}\right) .
$$

Thus we have

$$
\omega(\lambda, \mu)=-\frac{16}{\left(9+\lambda^{2}\right)\left(9+\mu^{2}\right)} \frac{J}{T}+\mathcal{O}\left(1 / T^{2}\right)
$$

\section{Computation of the 2-site density matrix}

Due to rotational invariance the density operator of two neighbouring spin-1 sites has to take the form

$$
D^{[2]}\left(\xi_{1}, \xi_{2}\right)=\rho_{1}\left(\xi_{1}, \xi_{2}\right) \mathbb{1}+\rho_{2}\left(\xi_{1}, \xi_{2}\right) P^{[2]}+\rho_{3}\left(\xi_{1}, \xi_{2}\right) P^{(0)},
$$

where $P^{[2]}$ is the permutation operator and $P^{(0)}:=(3 \times)$ the projector onto the singlet. Three coefficients $\rho_{k}, k=1,2,3$, have to be determined.

The general functional equation of $\mathrm{qKZ}$ type specialized to the 2-site case and written in terms of the above introduced coefficients reads

$$
\left(\begin{array}{l}
\rho_{1}\left(\xi_{1}-2 \mathrm{i}, \xi_{2}\right) \\
\rho_{2}\left(\xi_{1}-2 \mathrm{i}, \xi_{2}\right) \\
\rho_{3}\left(\xi_{1}-2 \mathrm{i}, \xi_{2}\right)
\end{array}\right)=L\left(\xi_{1}-\xi_{2}\right) \cdot\left(\begin{array}{l}
\rho_{1}\left(\xi_{1}, \xi_{2}\right) \\
\rho_{2}\left(\xi_{1}, \xi_{2}\right) \\
\rho_{3}\left(\xi_{1}, \xi_{2}\right)
\end{array}\right)
$$

Using the shorthand notation $\xi:=\xi_{1}-\xi_{2}$ the matrix $L(\xi)$ is given by

$$
L(\xi)=\left(\begin{array}{clc}
\frac{\xi^{2}\left(20+\xi^{2}\right)}{(\xi-2 \mathrm{i})(\xi+2 \mathrm{i})(\xi-4 \mathrm{i})(\xi+4 \mathrm{i})} & \frac{4 \mathrm{i} \xi\left(4-4 \mathrm{i} \xi+\xi^{2}\right)}{(\xi-2 \mathrm{i})(\xi+2 \mathrm{i})(\xi-4 \mathrm{i})(\xi+4 \mathrm{i})} & \frac{4 \mathrm{i} \xi}{(\xi+2 \mathrm{i})(\xi+4 \mathrm{i})} \\
-\frac{16 \xi^{2}}{(\xi-2 \mathrm{i})(\xi+2 \mathrm{i})(\xi-4 \mathrm{i})(\xi+4 \mathrm{i})} & \frac{-4 \mathrm{i}\left(8-6 \mathrm{i} \xi+\xi^{2}\right)}{(\xi-2 \mathrm{i})(\xi+2 \mathrm{i})(\xi-4 \mathrm{i})(\xi+4 \mathrm{i})} & \frac{\xi(\xi-2 \mathrm{i})}{(\xi+2 \mathrm{i})(\xi+4 \mathrm{i})} \\
\frac{16\left(12+\xi^{2}\right)}{(\xi-2 \mathrm{i})(\xi+2 \mathrm{i})(\xi-4 \mathrm{i})(\xi+4 \mathrm{i})} & \frac{64-16 \mathrm{i} \xi-4 \xi^{2}-8 \mathrm{i} \xi^{3}+\xi^{4}}{(\xi-2 \mathrm{i})(\xi+2 \mathrm{i})(\xi-4 \mathrm{i})(\xi+4 \mathrm{i})} & \frac{-4 \mathrm{i}(\xi-2 \mathrm{i})}{(\xi+2 \mathrm{i})(\xi+4 \mathrm{i})}
\end{array}\right)
$$

The functional equations for the three coefficients may be disentangled to some degree by the transformation

$$
\left(\begin{array}{l}
F \\
G \\
H
\end{array}\right)\left(\xi_{1}, \xi_{2}\right)=\left(\begin{array}{ccc}
9 & 3 & 3 \\
-16 & -\xi^{2}-8 & \xi^{2}+4 \\
-8 & -\frac{1}{2} \xi^{2}-6 & \frac{1}{2} \xi^{2}-6
\end{array}\right)\left(\begin{array}{l}
\rho_{1} \\
\rho_{2} \\
\rho_{3}
\end{array}\right)\left(\xi_{1}, \xi_{2}\right) .
$$


In fact, $F$ is fixed by the trace condition of the density operator

$$
F\left(\xi_{1}, \xi_{2}\right)=9 \rho_{1}\left(\xi_{1}, \xi_{2}\right)+3 \rho_{2}\left(\xi_{1}, \xi_{2}\right)+3 \rho_{3}\left(\xi_{1}, \xi_{2}\right)=\operatorname{tr} D^{[2]}\left(\xi_{1}, \xi_{2}\right) \equiv 1 .
$$

Hence we find the simpler looking functional equations

$$
\left(\begin{array}{c}
1 \\
G \\
H
\end{array}\right)_{\text {shift }}=\left(\begin{array}{ccc}
1 & 0 & 0 \\
0 & -\frac{\xi(\xi-6 \mathrm{i})}{(\xi-2 \mathrm{i})(\xi+4 \mathrm{i})} & 0 \\
-\frac{256 i(\xi-\mathrm{i})}{3(\xi+2 \mathrm{i})(\xi-2 \mathrm{i})^{2}(\xi+4 \mathrm{i})} & -\frac{\xi\left(\xi-6 \mathrm{i}\left(\xi \xi^{2}-2 \mathrm{i} \xi-4\right)\right.}{(\xi-2 \mathrm{i})^{2}(\xi+2 \mathrm{i})(\xi+4 \mathrm{i})} & \frac{\xi^{2}(\xi-6 \mathrm{i})(\xi-4 \mathrm{i})}{(\xi-2 \mathrm{i})^{2}(\xi+2 \mathrm{i})(\xi+4 \mathrm{i})}
\end{array}\right)\left(\begin{array}{c}
1 \\
G \\
H
\end{array}\right),
$$

where the functions $G, H$ on the left hand side are taken at shifted arguments $\left(\xi_{1}-2 \mathrm{i}, \xi_{2}\right)$, and those on the right hand side at $\left(\xi_{1}, \xi_{2}\right)$. The inverse transformation is

$$
\left(\begin{array}{l}
\rho_{1}\left(\xi_{1}, \xi_{2}\right) \\
\rho_{2}\left(\xi_{1}, \xi_{2}\right) \\
\rho_{3}\left(\xi_{1}, \xi_{2}\right)
\end{array}\right)=\left(\begin{array}{ccc}
\frac{5 \xi^{2}+36}{45\left(\xi^{2}+4\right)} & -\frac{\xi^{2}}{30\left(\xi^{2}+4\right)} & \frac{\xi^{2}+6}{15\left(\xi^{2}+4\right)} \\
-\frac{64}{45\left(\xi^{2}+4\right)} & \frac{3 \xi^{2}-20}{60\left(\xi^{2}+4\right)} & -\frac{3 \xi^{2}+28}{30\left(\xi^{2}+4\right)} \\
\frac{16}{45\left(\xi^{2}+4\right)} & \frac{3 \xi^{2}+20}{60\left(\xi^{2}+4\right)} & -\frac{3 \xi^{2}+8}{30\left(\xi^{2}+4\right)}
\end{array}\right)\left(\begin{array}{c}
1 \\
G\left(\xi_{1}, \xi_{2}\right) \\
H\left(\xi_{1}, \xi_{2}\right)
\end{array}\right)
$$

Below, we show that the explicit and unique solution for the functions $\rho_{j}\left(\xi_{1}, \xi_{2}\right)$, $j=1,2,3$, is

$$
\begin{aligned}
\rho_{1}\left(\xi_{1}, \xi_{2}\right)=- & \frac{1}{N\left(\xi_{1}\right) N\left(\xi_{2}\right)} \frac{1}{180 \xi^{2}\left(4+\xi^{2}\right)}\left\{-\frac{9}{4}\left(5 \xi^{2}\left(4+\xi^{2}\right)\right)\right. \\
& -\frac{3}{2}\left(5 \xi^{2}\left(4+\xi^{2}\right)-96\right)\left(\omega\left(\xi_{1}-\mathrm{i}, \xi_{1}+\mathrm{i}\right)+\omega\left(\xi_{2}-\mathrm{i}, \xi_{2}+\mathrm{i}\right)\right) \\
& -\left(5 \xi^{2}\left(4+\xi^{2}\right)-96\right)\left(\omega\left(\xi_{1}-\mathrm{i}, \xi_{1}+\mathrm{i}\right) \omega\left(\xi_{2}-\mathrm{i}, \xi_{2}+\mathrm{i}\right)\right) \\
& -3\left(4+\xi^{2}\right)\left((\xi+2 \mathrm{i})\left(\xi_{-}-6 \mathrm{i}\right) \omega\left(\xi_{1}-\mathrm{i}, \xi_{2}+\mathrm{i}\right)+\left(\xi_{-2}-2 \mathrm{i}\right)\left(\xi_{1}+6 \mathrm{i}\right) \omega\left(\xi_{1}+\mathrm{i}, \xi_{2}-\mathrm{i}\right)\right) \\
& +3 \xi^{2}\left(16+\xi^{2}\right)\left(\omega\left(\xi_{1}-\mathrm{i}, \xi_{2}-\mathrm{i}\right)+\omega\left(\xi_{1}+\mathrm{i}, \xi_{2}+\mathrm{i}\right)\right) \\
& +\left(6+\xi^{2}\right)\left(\xi^{2}\left(16+\xi^{2}\right) \omega\left(\xi_{1}-\mathrm{i}, \xi_{2}-\mathrm{i}\right) \omega\left(\xi_{1}+\mathrm{i}, \xi_{2}+\mathrm{i}\right)\right. \\
& \left.\left.-\left(4+\xi^{2}\right)^{2} \omega\left(\xi_{1}-\mathrm{i}, \xi_{2}+\mathrm{i}\right) \omega\left(\xi_{1}+\mathrm{i}, \xi_{2}-\mathrm{i}\right)\right)\right\} \\
\rho_{2}\left(\xi_{1}, \xi_{2}\right)=- & \frac{1}{N\left(\xi_{1}\right) N\left(\xi_{2}\right)} \frac{1}{180 \xi^{2}\left(4+\xi^{2}\right)}\{ \\
& 12\left(5 \xi^{2}-28\right)\left(\omega\left(\xi_{1}-\mathrm{i}, \xi_{1}+\mathrm{i}\right)+\omega\left(\xi_{2}-\mathrm{i}, \xi_{2}+\mathrm{i}\right)\right) \\
& +8\left(5 \xi^{2}-28\right)\left(\omega\left(\xi_{1}-i, \xi_{1}+i\right) \omega\left(\xi_{2}-\mathrm{i}, \xi_{2}+\mathrm{i}\right)\right) \\
& -3\left(4+\xi^{2}\right)\left(\left(\xi^{2}+2 \mathrm{i}\right)\left(\xi_{1}+14 \mathrm{i}\right) \omega\left(\xi_{1}-\mathrm{i}, \xi_{2}+\mathrm{i}\right)+\left(\xi_{-2} \mathrm{i}\right)\left(\xi_{-1}-14 \mathrm{i}\right) \omega\left(\xi_{1}+\mathrm{i}, \xi_{2}-\mathrm{i}\right)\right) \\
& -12 \xi^{2}\left(16+\xi^{2}\right)\left(\omega\left(\xi_{1}-\mathrm{i}, \xi_{2}-\mathrm{i}\right)+\omega\left(\xi_{1}+\mathrm{i}, \xi_{2}+\mathrm{i}\right)\right) \\
& -\frac{1}{2}\left(28+3 \xi^{2}\right)\left(\xi^{2}\left(16+\xi^{2}\right) \omega\left(\xi_{1}-\mathrm{i}, \xi_{2}-\mathrm{i}\right) \omega\left(\xi_{1}+\mathrm{i}, \xi_{2}+\mathrm{i}\right)\right. \\
& \left.\left.-\left(4+\xi^{2}\right)^{2} \omega\left(\xi_{1}-\mathrm{i}, \xi_{2}+\mathrm{i}\right) \omega\left(\xi_{1}+\mathrm{i}, \xi_{2}-\mathrm{i}\right)\right)\right\} \\
\rho_{3}\left(\xi_{1}, \xi_{2}\right)=- & \frac{1}{N\left(\xi_{1}\right) N\left(\xi_{2}\right)} \frac{180 \xi^{2}\left(4+\xi^{2}\right)\{}{(89 \mathrm{~b})} \\
& 1
\end{aligned}
$$




$$
\begin{aligned}
& -12\left(8+5 \xi^{2}\right)\left(\omega\left(\xi_{1}-i, \xi_{1}+i\right)+\omega\left(\xi_{2}-i, \xi_{2}+i\right)\right) \\
& -8\left(8+5 \xi^{2}\right)\left(\omega\left(\xi_{1}-i, \xi_{1}+i\right) \omega\left(\xi_{2}-i, \xi_{2}+i\right)\right) \\
& +12\left(4+\xi^{2}\right)\left((\xi-i)\left(\xi_{1}+2 i\right) \omega\left(\xi_{1}-i, \xi_{2}+i\right)+(\xi-2 i)(\xi+i) \omega\left(\xi_{1}+i, \xi_{2}-i\right)\right) \\
& +3 \xi^{2}\left(16+\xi^{2}\right)\left(\omega\left(\xi_{1}-i, \xi_{2}-i\right)+\omega\left(\xi_{1}+i, \xi_{2}+i\right)\right) \\
& -\frac{1}{2}\left(8+3 \xi^{2}\right)\left(\xi^{2}\left(16+\xi^{2}\right) \omega\left(\xi_{1}-i, \xi_{2}-i\right) \omega\left(\xi_{1}+i, \xi_{2}+i\right)\right. \\
& \left.\left.-\left(4+\xi^{2}\right)^{2} \omega\left(\xi_{1}-i, \xi_{2}+i\right) \omega\left(\xi_{1}+i, \xi_{2}-i\right)\right)\right\}
\end{aligned}
$$

Note that the function $N(\xi)$ appearing in the denominator is defined in (72).

Proof: The symmetry of the function $\omega$

$$
\omega\left(\xi_{1}, \xi_{2}\right)=\omega\left(\xi_{2}, \xi_{1}\right)
$$

directly leads to the symmetry of the coefficients $\rho_{k}\left(\xi_{1}, \xi_{2}\right)$. The asymptotics of the function $\omega$

$$
\lim _{\xi_{j} \rightarrow \infty} \omega\left(\xi_{1}, \xi_{2}\right)=0 \quad \text { for any } j=1,2,
$$

as obtained from (26), for instance, entails the asymptotics of the functions $\rho_{k}\left(\xi_{1}, \xi_{2}\right)$

$$
\lim _{\xi_{j} \rightarrow \infty} \rho_{k}\left(\xi_{1}, \xi_{2}\right)= \begin{cases}\frac{1}{9} & \text { for } k=1, \\ 0 & \text { else. }\end{cases}
$$

The functions $\rho_{k}\left(\xi_{1}, \xi_{2}\right)$ as solutions of the functional equations were constructed in the following way: (74) is independent of the second spectral parameter, hence also the function

$$
\begin{aligned}
& g\left(\xi_{1}, \xi_{2}\right):=\frac{C}{N\left(\xi_{1}\right) N\left(\xi_{2}\right)} \\
&\left\{\Omega\left(\xi_{1}+\mathrm{i}, \xi_{2}+\mathrm{i}\right)+\Omega\left(\xi_{1}-\mathrm{i}, \xi_{2}+\mathrm{i}\right)+\Omega\left(\xi_{1}+\mathrm{i}, \xi_{2}-\mathrm{i}\right)+\Omega\left(\xi_{1}-\mathrm{i}, \xi_{2}-\mathrm{i}\right)\right. \\
&\left.-o\left(\xi_{1}-\xi_{2}-\mathrm{i}\right)-o\left(\xi_{1}-\xi_{2}+\mathrm{i}\right)\right\}
\end{aligned}
$$

satisfies (74), where $C$ is some constant. Then it is easy to show that the function

$$
\begin{array}{r}
h\left(\xi_{1}, \xi_{2}\right):=\frac{C}{N\left(\xi_{1}\right) N\left(\xi_{2}\right)}\left\{\Omega\left(\xi_{1}+\mathrm{i}, \xi_{2}+\mathrm{i}\right) \Omega\left(\xi_{1}-\mathrm{i}, \xi_{2}-\mathrm{i}\right)-\Omega\left(\xi_{1}-\mathrm{i}, \xi_{2}+\mathrm{i}\right) \Omega\left(\xi_{1}+\mathrm{i}, \xi_{2}-\mathrm{i}\right)\right. \\
\left.+\Omega\left(\xi_{1}-\mathrm{i}, \xi_{2}+\mathrm{i}\right) o\left(\xi_{1}-\xi_{2}+\mathrm{i}\right)+\Omega\left(\xi_{1}+\mathrm{i}, \xi_{2}-\mathrm{i}\right) o\left(\xi_{1}-\xi_{2}-\mathrm{i}\right)-o\left(\xi_{1}-\xi_{2}+\mathrm{i}\right) o\left(\xi_{1}-\xi_{2}-\mathrm{i}\right)\right\}
\end{array}
$$

satisfies

$$
h\left(\xi_{1}, \xi_{2}\right)-h\left(\xi_{1}-2 \mathrm{i}, \xi_{2}\right)=o\left(\xi_{1}-\xi_{2}-\mathrm{i}\right) g\left(\xi_{1}, \xi_{2}\right)
$$


Define

$$
\begin{aligned}
G\left(\xi_{1}, \xi_{2}\right) & :=\frac{1}{2 \mathrm{i}}\left(\xi^{2}+16\right)\left(\xi^{2}+4\right) g\left(\xi_{1}, \xi_{2}\right), \\
H\left(\xi_{1}, \xi_{2}\right) & :=\frac{1}{(2 \mathrm{i})^{2}}\left(\xi^{2}+16\right)\left(\xi^{2}+4\right)^{2} h\left(\xi_{1}, \xi_{2}\right)+\frac{4}{3}\left(\frac{2 \mathrm{i}}{\xi}\right)^{2},
\end{aligned}
$$

then these functions satisfy (87). Applying the inverse transformation (88) the constant in (93) and (94) is fixed by the analyticity requirement for the functions $\rho_{k}\left(\xi_{1}, \xi_{2}\right)$, leading to the cancellation of spurious poles at $\xi_{1} \equiv \xi_{2}$. Using the identity (64) or (72) respectively leads to the results (89).

Finally, we consider the low and high temperature limits of $\rho_{k}\left(\xi_{1}, \xi_{2}\right)$ in the homogeneous case $\xi_{1}=\xi_{2}=0$. With the results we easily calculate any nearest neighbour correlators like for instance the internal energy $e=-T^{2} \frac{\partial}{\partial T}(f / T)$ by

$$
\left.e\right|_{h=0}=-\frac{3 J}{2}\left[2 \rho_{1}(0,0)+3 \rho_{3}(0,0)\right]
$$

Low temperature limit, zero field

By use of (77) we obtain

$$
\rho_{1}(0,0)=\frac{1}{2}-\frac{2}{45} \pi^{2}, \quad \rho_{2}(0,0)=-\frac{19}{18}+\frac{14}{135} \pi^{2}, \quad \rho_{3}(0,0)=-\frac{1}{9}+\frac{4}{135} \pi^{2} .
$$

Seemingly no zeta function values appear in these expressions. At first sight, this looks qualitatively different from the case of the spin-1/2 Heisenberg chain. Here, for the spin1 chain, we find a $\pi^{2}$ expression instead of zeta functions at odd integers. However, this can be rewritten in terms of Riemann's zeta function at the special argument $\zeta(2)=\frac{\pi^{2}}{6}$. We will see below that this pattern continues and for the 3 -site density matrix we obtain rational degree 3 polynomials in $\pi^{2}$ or equivalently linear expressions in $\zeta(2), \zeta(4)$ and $\zeta(6)$ with rational coefficients.

For the energy in the zero-temperature limit we simply get

$$
\left.e\right|_{h=0, T=0}=-J
$$

without any $\pi^{2}$ contributions. This result is in agreement with the literature, cf. [38].

High temperature asymptotics, zero field

By use of (81) we obtain

$$
\begin{aligned}
& \rho_{1}(0,0) \simeq \frac{1}{9}-\frac{1}{108} \frac{J}{T}+\mathcal{O}\left(T^{-2}\right), \\
& \rho_{2}(0,0) \simeq-\frac{1}{36} \frac{J}{T}+\mathcal{O}\left(T^{-2}\right), \\
& \rho_{3}(0,0) \simeq \frac{1}{18} \frac{J}{T}+\mathcal{O}\left(T^{-2}\right),
\end{aligned}
$$

and for the energy

$$
\left.e\right|_{h=0}=-\frac{J}{3}-\frac{2}{9} \frac{J^{2}}{T}+\mathcal{O}\left(T^{-2}\right)
$$




\section{Computation of the 3-site density matrix}

Similar to the 2-site case in the preceding section, the density operator of a 3-site segment is presented in the form,

$$
D^{[2]}\left(\xi_{1}, \xi_{2}, \xi_{3}\right)=\sum_{\alpha=1}^{15} \rho_{\alpha}\left(\xi_{1}, \xi_{2}, \xi_{3}\right) P_{\alpha}
$$

The projectors $P_{\alpha}(1 \leq \alpha \leq 15)$ are graphically represented in Fig. 4 . The lines connect-
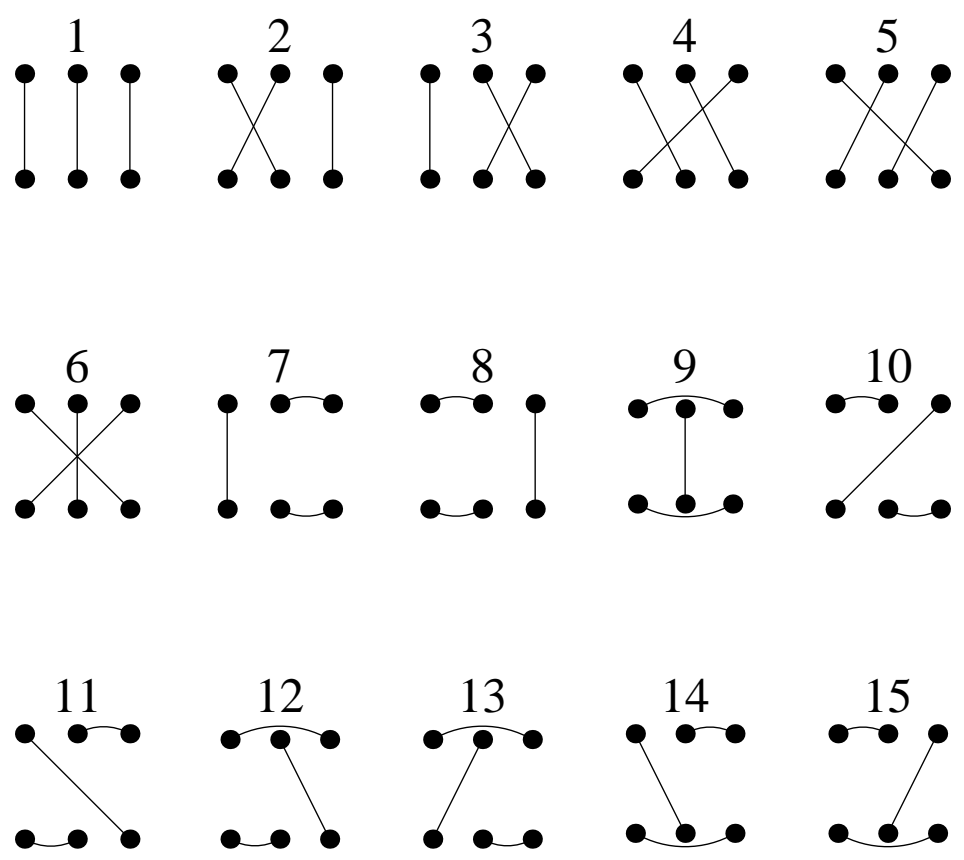

Figure 4:

ing upper and lower rows indicate Kronecker deltas. The lines connecting nodes $a$ and $b(1 \leq a<b \leq 3)$ in the same row represent matrix elements $C\left(i_{a}, i_{b}\right) \delta_{i_{a}+i_{b}, 0}$ such that

$$
C\left(i_{a}, i_{b}\right)= \begin{cases}1 & \left(i_{a}, i_{b}\right)=(0,0) \\ -1 & \left(i_{a}, i_{b}\right)=(1,-1),(-1,1) .\end{cases}
$$

This choice of projectors ensures the $S U(2)$ symmetry of the density operator,

$$
G D^{[2]}\left(\xi_{1}, \xi_{2}, \xi_{3}\right) G^{-1}=D^{[2]}\left(\xi_{1}, \xi_{2}, \xi_{3}\right), \quad G \in \mathrm{SU}(2) .
$$

For later convenience, we also introduce renormalized coefficients

$$
\widetilde{\rho}_{a}\left(\xi_{1}, \xi_{2}, \xi_{3}\right)=\prod_{j=1}^{3} N\left(\xi_{j}\right) \rho_{a}\left(\xi_{1}, \xi_{2}, \xi_{3}\right),
$$

where $N\left(\xi_{j}\right)$ is defined in (72). 
The result of our calculations takes a compact form in the homogeneous limit $\xi_{1}=\xi_{2}=\xi_{3}=0$ at $T=0$ (where $\left.\rho_{a}=8 \widetilde{\rho}_{a}\right)$ :

$$
\begin{aligned}
& \widetilde{\rho}_{1}=\frac{1879}{432}-\frac{3497}{1350} \pi^{2}+\frac{53}{135} \pi^{4}-\frac{11296}{637875} \pi^{6} \\
& \widetilde{\rho}_{2}=\widetilde{\rho}_{3}=-\frac{953}{2700} \pi^{2}+\frac{37}{675} \pi^{4}+\frac{1043}{2160}-\frac{1552}{637875} \pi^{6} \\
& \widetilde{\rho}_{4}=\widetilde{\rho}_{5}=\frac{983}{900} \pi^{2}-\frac{251}{144}+\frac{1592}{212625} \pi^{6}-\frac{1}{6} \pi^{4} \\
& \widetilde{\rho}_{6}=\frac{197}{54} \pi^{2}-\frac{374}{675} \pi^{4}-\frac{13021}{2160}+\frac{3184}{127575} \pi^{6} \\
& \widetilde{\rho}_{7}=\widetilde{\rho}_{8}=\frac{2189}{1350} \pi^{2}-\frac{166}{675} \pi^{4}-\frac{2917}{1080}+\frac{7072}{637875} \pi^{6} \\
& \widetilde{\rho}_{9}=\frac{641}{225} \pi^{2}-\frac{292}{675} \pi^{4}-\frac{5119}{1080}+\frac{12448}{637875} \pi^{6} \\
& \widetilde{\rho}_{10}=\widetilde{\rho}_{11}=-\frac{253}{135} \pi^{2}+\frac{371}{120}-\frac{544}{42525} \pi^{6}+\frac{64}{225} \pi^{4} \\
& \widetilde{\rho}_{12}=\widetilde{\rho}_{13}=\widetilde{\rho}_{14}=\widetilde{\rho}_{15}=\frac{343}{360}-\frac{751}{1350} \pi^{2}+\frac{19}{225} \pi^{4}-\frac{272}{70875} \pi^{6}
\end{aligned}
$$

Although at the intermediate stages nontrivial numbers like Euler's $\gamma$ occur, the final results are rational polynomials of degree 3 in $\pi^{2}$ and hence are simply expressible by use of Riemann's zeta function at even integers

$$
\zeta(2)=\frac{\pi^{2}}{6}, \quad \zeta(4)=\frac{\pi^{4}}{90}, \quad \zeta(6)=\frac{\pi^{6}}{945}
$$

In the remainder of this section we describe the computational strategy.

Firstly, we note that not all of coefficients are independent but some of them are related, reflecting the diagrammatic symmetry,

$$
\begin{aligned}
\rho_{5}\left(\xi_{1}, \xi_{2}, \xi_{3}\right) & =\rho_{4}\left(-\xi_{1},-\xi_{2},-\xi_{3}\right) & \rho_{11}\left(\xi_{1}, \xi_{2}, \xi_{3}\right) & =\rho_{10}\left(-\xi_{1},-\xi_{2},-\xi_{3}\right) \\
\rho_{15}\left(\xi_{1}, \xi_{2}, \xi_{3}\right) & =\rho_{12}\left(-\xi_{1},-\xi_{2},-\xi_{3}\right) & \rho_{14}\left(\xi_{1}, \xi_{2}, \xi_{3}\right) & =\rho_{13}\left(-\xi_{1},-\xi_{2},-\xi_{3}\right) \\
\rho_{3}\left(\xi_{1}, \xi_{2}, \xi_{3}\right) & =\rho_{2}\left(\xi_{3}, \xi_{2}, \xi_{1}\right) & \rho_{8}\left(\xi_{1}, \xi_{2}, \xi_{3}\right) & =\rho_{7}\left(\xi_{3}, \xi_{2}, \xi_{1}\right) \\
\rho_{14}\left(\xi_{1}, \xi_{2}, \xi_{3}\right) & =\rho_{12}\left(\xi_{3}, \xi_{2}, \xi_{1}\right) & \rho_{15}\left(\xi_{1}, \xi_{2}, \xi_{3}\right) & =\rho_{13}\left(\xi_{3}, \xi_{2}, \xi_{1}\right) .
\end{aligned}
$$

The first four relations hold due to the up-down symmetry and the last four ones due to the symmetry w.r.t. reflection at the anti-diagonal line. This reduces our task considerably. The explicit expressions of $\rho_{j}\left(\widetilde{\rho}_{j}\right)$ in terms of $\omega$ functions are still very much involved. Below we present the simplest case $\widetilde{\rho}_{1}$ for illustration. Supplementary arguments are given in Appendix D. The expression for $\widetilde{\rho}_{1}$ contains up to trilinear terms 
in $\omega$,

$$
\begin{aligned}
& \widetilde{\rho}_{1}\left(\xi_{1}, \xi_{2}, \xi_{3}\right)=\frac{N\left(\xi_{1}\right) N\left(\xi_{2}\right) N\left(\xi_{3}\right)}{27}+c_{1}^{(1)} \omega\left(\xi_{1}^{-}, \xi_{2}^{-}\right)+c_{2}^{(1)} \omega\left(\xi_{1}^{-}, \xi_{1}^{+}\right)+c_{3}^{(1)} \omega\left(\xi_{1}^{-}, \xi_{2}^{+}\right) \\
& +c_{1}^{(2)} \omega\left(\xi_{1}^{-}, \xi_{1}^{+}\right) \omega\left(\xi_{2}^{-}, \xi_{3}^{-}\right)+c_{2}^{(2)} \omega\left(\xi_{1}^{-}, \xi_{2}^{-}\right) \omega\left(\xi_{2}^{+}, \xi_{3}^{-}\right)+c_{3}^{(2)} \omega\left(\xi_{1}^{-}, \xi_{1}^{+}\right) \omega\left(\xi_{2}^{-}, \xi_{3}^{+}\right) \\
& +c_{4}^{(2)} \omega\left(\xi_{1}^{+}, \xi_{3}^{-}\right) \omega\left(\xi_{2}^{-}, \xi_{3}^{+}\right)+c_{5}^{(2)} \omega\left(\xi_{1}^{-}, \xi_{2}^{-}\right) \omega\left(\xi_{1}^{+}, \xi_{3}^{+}\right)+c_{6}^{(2)} \omega\left(\xi_{2}^{-}, \xi_{3}^{+}\right) \omega\left(\xi_{2}^{+}, \xi_{3}^{-}\right) \\
& +c_{7}^{(2)} \omega\left(\xi_{1}^{-}, \xi_{1}^{+}\right) \omega\left(\xi_{2}^{-}, \xi_{2}^{+}\right)+c_{8}^{(2)} \omega\left(\xi_{2}^{-}, \xi_{3}^{-}\right) \omega\left(\xi_{2}^{+}, \xi_{3}^{+}\right)+c_{1}^{(3)} \omega\left(\xi_{1}^{-}, \xi_{1}^{+}\right) \omega\left(\xi_{2}^{-}, \xi_{3}^{+}\right) \omega\left(\xi_{2}^{+}, \xi_{3}^{-}\right) \\
& +c_{2}^{(3)} \omega\left(\xi_{1}^{-}, \xi_{2}^{+}\right) \omega\left(\xi_{1}^{+}, \xi_{3}^{-}\right) \omega\left(\xi_{2}^{-}, \xi_{3}^{+}\right)+c_{3}^{(3)} \omega\left(\xi_{1}^{-}, \xi_{1}^{+}\right) \omega\left(\xi_{2}^{-}, \xi_{2}^{+}\right) \omega\left(\xi_{3}^{-}, \xi_{3}^{+}\right) \\
& +c_{4}^{(3)} \omega\left(\xi_{1}^{-}, \xi_{2}^{-}\right) \omega\left(\xi_{1}^{+}, \xi_{3}^{-}\right) \omega\left(\xi_{2}^{+}, \xi_{3}^{+}\right)+c_{5}^{(3)} \omega\left(\xi_{1}^{-}, \xi_{1}^{+}\right) \omega\left(\xi_{2}^{-}, \xi_{3}^{-}\right) \omega\left(\xi_{2}^{+}, \xi_{3}^{+}\right) \\
& + \text {permutations and negation. }
\end{aligned}
$$

We use the shorthand notation, $\xi_{j}^{ \pm}:=\xi_{j} \pm \mathrm{i}$. The last term contains the distinct terms under permutations of $\left\{\xi_{i}\right\}$ and negation of spectral parameters $\xi_{i} \rightarrow-\xi_{i}$. We regard "distinct" after applying the symmetry properties $\omega\left(\lambda_{i}, \lambda_{j}\right)=\omega\left(\lambda_{j}, \lambda_{i}\right)$ and $\omega\left(\lambda_{i}, \lambda_{j}\right)=$ $\omega\left(-\lambda_{i},-\lambda_{j}\right)$. The coefficients $c_{j}^{(i)}$ are rational functions of $\xi_{1}, \xi_{2}, \xi_{3}$ and their explicit forms are listed.

$$
\begin{aligned}
c_{1}^{(1)}\left(\xi_{1}, \xi_{2}, \xi_{3}\right) & =-\frac{\left(\xi_{12}^{2}+4^{2}\right)\left(7 \xi_{13}\left(\xi_{13}-2 \mathrm{i}\right) \xi_{23}\left(\xi_{23}-2 \mathrm{i}\right)+80 \xi_{12}^{2}+2432\right)}{1680\left(\xi_{12}-2 \mathrm{i}\right)\left(\xi_{12}+2 \mathrm{i}\right) \xi_{13}\left(\xi_{13}-2 \mathrm{i}\right) \xi_{23}\left(\xi_{23}-2 \mathrm{i}\right)} \\
c_{2}^{(1)}\left(\xi_{1}, \xi_{2}, \xi_{3}\right) & =\frac{-7\left(-\xi_{12} \xi_{23}+2\right)^{2}-7\left(\xi_{13} \xi_{23}+2\right)^{2}-7 \xi_{12} \xi_{13}\left(\xi_{12}^{2}+\xi_{13}^{2}+\xi_{23}^{2}+8\right)-2056}{35 \xi_{12}^{2}\left(\xi_{12}-2 \mathrm{i}\right)\left(\xi_{12}+2 \mathrm{i}\right) \xi_{13}^{2}\left(\xi_{13}-2 \mathrm{i}\right)\left(\xi_{13}+2 \mathrm{i}\right)} \\
c_{3}^{(1)}\left(\xi_{1}, \xi_{2}, \xi_{3}\right) & =\frac{\left(\xi_{12}+2 \mathrm{i}\right)\left(\xi_{12}-6 \mathrm{i}\right)\left(7\left(\xi_{13} \xi_{23}+2\right)^{2}+2 \xi_{12}\left(7 \mathrm{i} \xi_{13} \xi_{23}+40\left(\xi_{12}-4 \mathrm{i}\right)\right)+2084\right)}{1680 \xi_{12}^{2} \xi_{13}\left(\xi_{13}-2 \mathrm{i}\right) \xi_{23}\left(\xi_{23}+2 \mathrm{i}\right)} \\
c_{1}^{(2)}\left(\xi_{1}, \xi_{2}, \xi_{3}\right) & =\frac{\left(\xi_{23}+4 \mathrm{i}\right)\left(-\xi_{23}+4 \mathrm{i}\right) p_{1}^{(2)}\left(\xi_{1}, \xi_{2}, \xi_{3}\right)}{12600 \xi_{12}^{2}\left(\xi_{12}-2 \mathrm{i}\right)\left(\xi_{12}+2 \mathrm{i}\right) \xi_{13}^{2}\left(\xi_{13}-2 \mathrm{i}\right)\left(\xi_{13}+2 \mathrm{i}\right)\left(\xi_{23}-2 \mathrm{i}\right)\left(\xi_{23}+2 \mathrm{i}\right)} \\
c_{2}^{(2)}\left(\xi_{1}, \xi_{2}, \xi_{3}\right) & =-\frac{i\left(\xi_{12}-4 \mathrm{i}\right)\left(\xi_{12}+4 \mathrm{i}\right)\left(\xi_{23}-4 \mathrm{i}\right)\left(-6 e_{2}^{2}+832 e_{2}+e_{3}^{2}-50 \xi_{21}^{2} \xi_{23}^{2}-1520 \xi_{21} \xi_{23}-27328\right)}{3150 \xi_{12}\left(\xi_{12}-2 \mathrm{i}\right)\left(\xi_{12}+2 \mathrm{i}\right) \xi_{13}\left(\xi_{13}-2 \mathrm{i}\right)\left(\xi_{13}+2 \mathrm{i}\right) \xi_{23}^{2}} \\
c_{3}^{(2)}\left(\xi_{1}, \xi_{2}, \xi_{3}\right) & =\frac{\left(\xi_{23}+2 \mathrm{i}\right) p_{3}^{(2)}\left(\xi_{1}, \xi_{2}, \xi_{3}\right)}{12600 \xi_{12}^{2}\left(\xi_{12}-2 \mathrm{i}\right)\left(\xi_{12}+2 \mathrm{i}\right) \xi_{13}^{2}\left(\xi_{13}-2 \mathrm{i}\right)\left(\xi_{13}+2 \mathrm{i}\right) \xi_{23}^{2}} \\
c_{4}^{(2)}\left(\xi_{1}, \xi_{2}, \xi_{3}\right) & =\frac{\left(\xi_{13}-4 \mathrm{i}\right)\left(\xi_{23}+4 \mathrm{i}\right) p_{4}^{(2)}\left(\xi_{1}, \xi_{2}, \xi_{3}\right)}{3150 \mathrm{i} \xi_{12}^{2}\left(\xi_{12}+2 \mathrm{i}\right) \xi_{13}^{2} \xi_{23}^{2}} \\
c_{5}^{(2)}\left(\xi_{1}, \xi_{2}, \xi_{3}\right) & =\frac{\left(\xi_{12}-4 \mathrm{i}\right)\left(\xi_{12}+4 \mathrm{i}\right)\left(\xi_{13}-4 \mathrm{i}\right)\left(\xi_{13}+4 \mathrm{i}\right) p_{4}^{(2)}\left(-\xi_{2},-\xi_{3},-\xi_{1}\right)}{3150 \mathrm{i} \xi_{12}\left(\xi_{12}-2 \mathrm{i}\right)\left(\xi_{12}+2 \mathrm{i}\right) \xi_{13}\left(\xi_{13}-2 \mathrm{i}\right)\left(\xi_{13}+2 \mathrm{i}\right) \xi_{23}^{2}\left(\xi_{23}-2 \mathrm{i}\right)} \\
c_{6}^{(2)}\left(\xi_{1}, \xi_{2}, \xi_{3}\right) & =\frac{3}{2} c_{1}^{(3)}\left(\xi_{1}, \xi_{2}, \xi_{3}\right) \\
c_{7}^{(2)}\left(\xi_{1}, \xi_{2}, \xi_{3}\right) & =\frac{3}{2} c_{3}^{(3)}\left(\xi_{1}, \xi_{2}, \xi_{3}\right) \\
c_{8}^{(2)}\left(\xi_{1}, \xi_{2}, \xi_{3}\right) & =\frac{3}{2} c_{5}^{(3)}\left(\xi_{1}, \xi_{2}, \xi_{3}\right)
\end{aligned}
$$




$$
\begin{aligned}
c_{1}^{(3)}\left(\xi_{1}, \xi_{2}, \xi_{3}\right) & =\frac{p_{1}^{(3)}\left(\xi_{1}, \xi_{2}, \xi_{3}\right)\left(\xi_{23}-2 \mathrm{i}\right)\left(\xi_{23}+2 \mathrm{i}\right)}{37800 \xi_{12}^{2}\left(\xi_{12}-2 \mathrm{i}\right)\left(\xi_{12}+2 \mathrm{i}\right) \xi_{13}^{2}\left(\xi_{13}-2 \mathrm{i}\right)\left(\xi_{13}+2 \mathrm{i}\right) \xi_{23}^{2}} \\
c_{2}^{(3)}\left(\xi_{1}, \xi_{2}, \xi_{3}\right) & =-\frac{i\left(-6 e_{2}^{2}+432 e_{2}+e_{3}^{2}-5648\right)\left(\xi_{12}+4 \mathrm{i}\right)\left(\xi_{13}-4 \mathrm{i}\right)\left(\xi_{23}+4 \mathrm{i}\right)}{9450 \xi_{12}^{2} \xi_{13}^{2} \xi_{23}^{2}} \\
c_{3}^{(3)}\left(\xi_{1}, \xi_{2}, \xi_{3}\right) & =-\frac{4\left(105 e_{2}^{4}-840 e_{2}^{3}+23632 e_{2}^{2}+420 e_{2} e_{3}^{2}+345856 e_{2}-4172 e_{3}^{2}+2891776\right)}{4725 \xi_{12}^{2}\left(\xi_{12}-2 \mathrm{i}\right)\left(\xi_{12}+2 \mathrm{i}\right) \xi_{13}^{2}\left(\xi_{13}-2 \mathrm{i}\right)\left(\xi_{13}+2 \mathrm{i}\right) \xi_{23}^{2}\left(\xi_{23}-2 \mathrm{i}\right)\left(\xi_{23}+2 \mathrm{i}\right)} \\
c_{4}^{(3)}\left(\xi_{1}, \xi_{2}, \xi_{3}\right) & =\frac{i\left(-6 e_{2}^{2}+432 e_{2}+e_{3}^{2}-5648\right)\left(\xi_{12}-4 \mathrm{i}\right)\left(\xi_{12}+4 \mathrm{i}\right)\left(\xi_{13}-4 \mathrm{i}\right)\left(\xi_{23}-4 \mathrm{i}\right)\left(\xi_{23}+4 \mathrm{i}\right)}{9450 \xi_{12}\left(\xi_{12}-2 \mathrm{i}\right)\left(\xi_{12}+2 \mathrm{i}\right) \xi_{13}^{2} \xi_{23}\left(\xi_{23}-2 \mathrm{i}\right)\left(\xi_{23}+2 \mathrm{i}\right)} \\
c_{5}^{(3)}\left(\xi_{1}, \xi_{2}, \xi_{3}\right) & =\frac{p_{1}^{(3)}\left(\xi_{1}, \xi_{2}, \xi_{3}\right)\left(\xi_{23}+4 \mathrm{i}\right)\left(-\xi_{23}+4 \mathrm{i}\right)}{37800 \xi_{12}^{2}\left(\xi_{12}-2 \mathrm{i}\right)\left(\xi_{12}+2 \mathrm{i}\right) \xi_{13}^{2}\left(\xi_{13}-2 \mathrm{i}\right)\left(\xi_{13}+2 \mathrm{i}\right)\left(\xi_{23}-2 \mathrm{i}\right)\left(\xi_{23}+2 \mathrm{i}\right)}
\end{aligned}
$$

where $e_{2}$ and $e_{3}$ are elementary symmetric functions, defined as coeffcients of $t^{2}$ and $t^{3}$ in the expansion of $\left(1+t \xi_{12}\right)\left(1+t \xi_{23}\right)\left(1+t \xi_{31}\right)$. The nontrivial polynomials in the numerators are defined by

$$
\begin{aligned}
p_{1}^{(2)}\left(\xi_{1}, \xi_{2}, \xi_{3}\right) & =1748992+384 e_{2}^{2}+53248 e_{2}+106496 \xi_{23}^{2} \\
& +\xi_{12} \xi_{13}\left(-476 e_{2} \xi_{12} \xi_{13}+35 \xi_{12}^{3} \xi_{13}^{3}-196 \xi_{12}^{2} \xi_{13}^{2}+12720 \xi_{12} \xi_{13}-4800 \xi_{23}^{2}-136704\right) \\
p_{3}^{(2)}\left(\xi_{1}, \xi_{2}, \xi_{3}\right) & =-5783552 \mathrm{i}-606208 \xi_{23}+384 e_{2}^{2} \xi_{23}-2304 \mathrm{i} e_{2}^{2} \\
& +\xi_{12}^{2} \xi_{13}^{2}\left(\xi_{23}-6 \mathrm{i}\right)\left(-476 e_{2}+35 \xi_{12}^{2} \xi_{13}^{2}-196 \xi_{12} \xi_{13}\right)-9728 e_{2} \xi_{23}+232448 \mathrm{i} e_{2} \\
& -80 \xi_{12}^{2} \xi_{13}^{2}\left(37 \xi_{23}+562 \mathrm{i}\right)-320 \xi_{12} \xi_{13}\left(15 \xi_{23}^{3}-90 i \xi_{23}^{2}-76 \xi_{23}-2888 \mathrm{i}\right) \\
p_{4}^{(2)}\left(\xi_{1}, \xi_{2}, \xi_{3}\right) & =22592-3280 i \xi_{12}-6 e_{2}^{2}-88 e_{2}-60 i \xi_{12}\left(\xi_{13}^{2}+\xi_{23}^{2}\right) \\
& +\xi_{31} \xi_{32}\left(1640+\left(\xi_{21}^{2}+10 \mathrm{i} \xi_{12}+30\right) \xi_{31} \xi_{32}\right) \\
p_{1}^{(3)}\left(\xi_{1}, \xi_{2}, \xi_{3}\right) & =5783552-384\left(e_{2}^{3}+3 e_{3}^{2}\right)+e_{2}^{2}\left(140 \xi_{12}^{2} \xi_{13}^{2}+33792\right)+5184 e_{2} \xi_{12} \xi_{13} \xi_{23}^{2}-803840 e_{2} \\
& +\xi_{12} \xi_{13}\left(\xi_{12} \xi_{13}\left(35 e_{3}^{2}+336 \xi_{23}^{4}+13304 \xi_{23}^{2}+13472\right)\right. \\
& \left.+70 \xi_{12}^{3} \xi_{13}^{3}+1296 \xi_{12}^{2} \xi_{13}^{2}-169728 \xi_{23}^{2}-1495552\right) .
\end{aligned}
$$

The above result is checked against direct computations (of some density matrix elements ) for fixed Trotter numbers.

\section{Conclusion}

The present report offers a novel way to approach the exact evaluation of correlation functions of higher spin $s u(2)$ chains in a quantitative manner. It utilizes the discrete functional relations of $\mathrm{qKZ}$ equation type, as well as the direct fusion procedure. Explicit results for shorter segments $(m=2,3)$ clearly show an improvement of the present approach compared to the former formulation in [16]. The correlation functions are not given by complex contour integrals but they are given in simple factorized forms, using rational functions of inhomogeneities and only one nontrivial ingredient $\omega$. 
One of the direct consequences of such nice expressions is exemplified in Section 5, and Section 6: correlation functions of the $S=1$ chain contain only Riemann's zeta function with even integer arguments. This complements the famous conjecture in [8] that correlation functions of the $S=\frac{1}{2}$ chain are given in terms of zeta functions with odd integer arguments.

Clearly many subjects are still left open. The appearance of the zeta function with odd integer arguments for the $S=\frac{1}{2}$ chain is naturally explained via the explicit construction of the solution to the qKZ equation using $q$-oscillators. We believe that an analogous reasoning is necessary and possible for the higher spin case.

It becomes clearer that bulk quantities (e.g. the specific heat) of some novel materials, e.g., spin-ladders and spin-nanotubes are described by models with higher rank Lie symmetry. Their quantum correlations are definitely the next promising goal of our understanding of these materials. Therefore the extension of the present approach to the $\operatorname{su}(n)$ case is highly desirable.

We hope to come back to these issues in the near future.

Acknowledgment. We would like to thank B. Aufgebauer, H. Boos, F. Göhmann, M. Jimbo, A. Kuniba, T. Miwa for stimulating discussions. AK is grateful to Shizuoka University for hospitality. His work was supported by JSPS. JS is supported by a Grant-in-Aid for Scientific Research No. 20540370.

\section{Appendix A: Auxiliary functions for spin 1}

It is sometimes more convenient to deal with polynomials rather than with rational

functions. For this reason a different normalization of the elementary $R$-matrix was used in [34]. This leads to differently normalized transfer matrix eigenvalues. In order to simplify the comparison with [34] we define the functions

$$
\begin{aligned}
\Lambda_{1}(\lambda)= & \phi_{-}(\lambda-3 i) \phi_{+}(\lambda+3 i) \varphi_{-}(\lambda-2 i) \varphi_{+}(\lambda+2 i) \cdot \Lambda^{[1]}(\lambda), \\
\Lambda_{2}(\lambda)= & \phi_{-}(\lambda-4 i) \phi_{+}(\lambda+2 i) \phi_{-}(\lambda-2 i) \phi_{+}(\lambda+4 i) . \\
& \varphi_{-}(\lambda-3 i) \varphi_{+}(\lambda+i) \varphi_{-}(\lambda-i) \varphi_{+}(\lambda+3 i) \cdot \Lambda^{[2]}(\lambda) .
\end{aligned}
$$

Then, following [34], we introduce

$$
\begin{aligned}
& \lambda_{1}(\lambda)=\mathrm{e}^{-\frac{2 h}{T}} \phi(\lambda-\mathrm{i}) \phi(\lambda-3 \mathrm{i}) \varphi(\lambda) \varphi(\lambda-2 \mathrm{i}) \frac{q(\lambda+3 \mathrm{i})}{q(\lambda-\mathrm{i})} \\
& \lambda_{2}(\lambda)=\phi(\lambda+\mathrm{i}) \phi(\lambda-\mathrm{i}) \varphi^{2}(\lambda) \frac{q(\lambda-3 \mathrm{i}) q(\lambda+3 \mathrm{i})}{q(\lambda-\mathrm{i}) q(\lambda+\mathrm{i})} \\
& \lambda_{3}(\lambda)=\mathrm{e}^{\frac{2 h}{T}} \phi(\lambda+3 \mathrm{i}) \phi(\lambda+\mathrm{i}) \varphi(\lambda+2 \mathrm{i}) \varphi(\lambda) \frac{q(\lambda-3 \mathrm{i})}{q(\lambda+\mathrm{i})}
\end{aligned}
$$

where

$$
\phi(\lambda):=\phi_{+}(\lambda+\mathrm{i}) \phi_{-}(\lambda-\mathrm{i}), \varphi(\lambda):=\varphi_{+}(\lambda+\mathrm{i}) \varphi_{-}(\lambda-\mathrm{i}) .
$$

It follows that

$$
\Lambda_{2}(\lambda)=\lambda_{1}(\lambda)+\lambda_{2}(\lambda)+\lambda_{3}(\lambda)
$$


The basic auxiliary functions for spin 1 are

$$
\mathfrak{b}(\lambda)=\frac{\lambda_{1}(\lambda+\mathrm{i})+\lambda_{2}(\lambda+\mathrm{i})}{\lambda_{3}(\lambda+\mathrm{i})}, \quad \overline{\mathfrak{b}}(\lambda)=\frac{\lambda_{2}(\lambda-\mathrm{i})+\lambda_{3}(\lambda-\mathrm{i})}{\lambda_{1}(\lambda-\mathrm{i})},
$$

with corresponding capital functions

$$
\mathfrak{B}(\lambda)=1+\mathfrak{b}(\lambda), \quad \overline{\mathfrak{B}}(\lambda)=1+\overline{\mathfrak{b}}(\lambda) .
$$

In [34] the nonlinear integral equations (48) were derived from a set of functional equations satisfied by the functions $\mathfrak{b}, \overline{\mathfrak{b}}, \mathfrak{B}, \overline{\mathfrak{B}}$ together with

$$
y(\lambda)=\frac{\Lambda_{2}(\lambda)}{\phi(\lambda-3 \mathrm{i}) \phi(\lambda+3 \mathrm{i}) \varphi(\lambda-2 \mathrm{i}) \varphi(\lambda+2 \mathrm{i})}, \quad Y(\lambda)=1+y(\lambda) .
$$

In appendix $B$ we present a derivation of integral equations by use of algebraic relations exposed below.

$$
\begin{aligned}
\mathfrak{b}(\lambda) & =\mathrm{e}^{-\frac{3 h}{T}} \Lambda_{1}(\lambda) \frac{\phi(\lambda)}{\phi(\lambda+4 \mathrm{i}) \phi(\lambda+2 \mathrm{i}) \varphi(\lambda+3 \mathrm{i})} \frac{q(\lambda+4 \mathrm{i})}{q(\lambda-2 \mathrm{i})} \\
\overline{\mathfrak{b}}(\lambda) & =\mathrm{e}^{\frac{3 h}{T}} \Lambda_{1}(\lambda) \frac{\phi(\lambda)}{\phi(\lambda-4 \mathrm{i}) \phi(\lambda-2 \mathrm{i}) \varphi(\lambda-3 \mathrm{i})} \frac{q(\lambda-4 \mathrm{i})}{q(\lambda+2 \mathrm{i})} \\
\Lambda_{2}(\lambda) & =\mathfrak{B}(\lambda-\mathrm{i}) \lambda_{3}(\lambda)=\overline{\mathfrak{B}}(\lambda+\mathrm{i}) \lambda_{1}(\lambda) \\
Y(\lambda) & =\frac{\Lambda_{1}(\lambda-\mathrm{i}) \Lambda_{1}(\lambda+\mathrm{i})}{\phi(\lambda-3 \mathrm{i}) \phi(\lambda+3 \mathrm{i}) \varphi(\lambda-2 \mathrm{i}) \varphi(\lambda+2 \mathrm{i})}
\end{aligned}
$$

Note that from (A.7) and (A.8c) we obtain

$$
1+y(\lambda)^{-1}=\frac{Y(\lambda)}{y(\lambda)}=\frac{\Lambda_{1}(\lambda+\mathrm{i}) \Lambda_{1}(\lambda-\mathrm{i})}{\Lambda_{2}(\lambda)}
$$

\section{Appendix B: NLIE with straight contour integrations}

To proceed further, it is convenient to consider equations in Fourier space. For a smooth function $f(\lambda)$ we define

$$
\hat{f}(k)=\int_{-\infty}^{\infty} \frac{\mathrm{d} \lambda}{2 \pi} \mathrm{e}^{\mathrm{i} k \lambda} f(\lambda), \quad \operatorname{dlf}(k)=\int_{-\infty}^{\infty} \frac{\mathrm{d} \lambda}{2 \pi} \mathrm{e}^{\mathrm{i} k \lambda}\left(\frac{d}{d \lambda} \log f(\lambda)\right) .
$$

Equations (A.8c), (A.2a), (A.2c) and (A.8d), after taking the logarithmic derivative and the Fourier transform, read

$$
\begin{aligned}
\mathrm{d} l \Lambda_{2} & =\mathrm{e}^{-k} \mathrm{dlB}+\mathrm{dl} \lambda_{3}=\mathrm{e}^{k} \mathrm{dl} \overline{\mathrm{B}}+\mathrm{dl} \lambda_{1}, \\
\mathrm{~d} l \lambda_{1} & =\left(\mathrm{e}^{-k}+\mathrm{e}^{-3 k}\right) \mathrm{dl} \phi_{-}+\mathrm{dl} \varphi_{0}+\mathrm{e}^{-2 k} \mathrm{dl} \varphi_{-}+\mathrm{e}^{3 k} \mathrm{dlq}_{+}-\mathrm{e}^{-k} \mathrm{dl} \mathrm{q}_{-}, \\
\mathrm{d} \mathrm{l} \lambda_{3} & =\left(\mathrm{e}^{k}+\mathrm{e}^{3 k}\right) \mathrm{dl} \phi_{+}+\mathrm{dl} \varphi_{0}+\mathrm{e}^{2 k} \mathrm{dl} \varphi_{+}+\mathrm{e}^{-3 k} \mathrm{dlq}_{-}-\mathrm{e}^{k} \mathrm{dll} \mathrm{q}_{+}, \\
\mathrm{dl} \mathrm{Y} & =\left(\mathrm{e}^{k}+\mathrm{e}^{-k}\right) \mathrm{dl} \Lambda_{1}-\mathrm{e}^{-3 k} \mathrm{dl} \phi_{-}-\mathrm{e}^{3 k} \mathrm{dl} \phi_{+}-\mathrm{e}^{-2 k} \mathrm{dl} \varphi_{-}-\mathrm{e}^{2 k} \mathrm{dl} \varphi_{+} .
\end{aligned}
$$

These equations simplify in different ways depending on the sign of $k$. 
$k>0$.

Here $\mathrm{dl} \phi_{+}=\mathrm{dl} \varphi_{+}=\mathrm{dlq} q_{+}=0$, hence from (B.2a)-(B.2c)

$$
\begin{aligned}
& \mathrm{d} l \lambda_{3}-\mathrm{d} l \lambda_{1}= \\
& -\left(\mathrm{e}^{-k}+\mathrm{e}^{-3 k}\right) \mathrm{dl} \phi_{-}-\mathrm{e}^{-2 k} \mathrm{dl} \varphi_{-}+\left(\mathrm{e}^{-k}+\mathrm{e}^{-3 k}\right) \mathrm{dl} \mathrm{q}_{-}=-\mathrm{e}^{-k} \mathrm{dlB}+\mathrm{e}^{k} \mathrm{dl} \overline{\mathrm{B}},
\end{aligned}
$$

from which we can express dlq $q_{-}$in terms of dl $\phi_{-}$, dl $\varphi_{-}$, dlB and dlB̈. From (B.2d) we obtain $\mathrm{dl} \Lambda_{1}$ in terms of $\mathrm{dl} \phi_{-}, \mathrm{dl} \varphi_{-}$and dlY. These expressions are then inserted into the Fourier transforms of the logarithmic derivatives of (A.8a) and (A.8d) yielding

$$
\begin{aligned}
& \mathrm{dlb}=\mathrm{dl} \phi_{0}+\frac{1}{\mathrm{e}^{k}+\mathrm{e}^{-k}}\left(-\mathrm{e}^{-k} \mathrm{dl} \phi_{-}+\mathrm{dl} \mathrm{Y}+\mathrm{e}^{-k} \mathrm{dlB}-\mathrm{e}^{k} \mathrm{dl} \overline{\mathrm{B}}\right), \\
& \mathrm{dly}=\mathrm{dl} \varphi_{0}+\frac{1}{\mathrm{e}^{k}+\mathrm{e}^{-k}}\left(-\mathrm{e}^{-k} \mathrm{dl} \varphi_{-}+\mathrm{dlB}+\mathrm{dl} \overline{\mathrm{B}}\right) .
\end{aligned}
$$

The explicit $\phi$ and $\varphi$ terms yield (note that dl $\phi_{-}$refers to the Fourier transform of the logarithmic derivative of $\phi(\lambda)$ in the lower half plane, it is not the Fourier transform of $\left.\phi_{-}(\lambda)\right)$ :

$$
\begin{aligned}
\mathrm{dl} \phi_{0}-\frac{\mathrm{e}^{-k}}{\mathrm{e}^{k}+\mathrm{e}^{-k}} \mathrm{dl} \phi_{-} & =-\mathrm{i} \frac{N}{2} \frac{\mathrm{e}^{u k}-\mathrm{e}^{-u k}}{\mathrm{e}^{k}+\mathrm{e}^{-k}}, \\
\mathrm{dl} \varphi_{0}-\frac{\mathrm{e}^{-k}}{\mathrm{e}^{k}+\mathrm{e}^{-k}} \mathrm{dl} \varphi_{-} & =-\mathrm{i} \frac{1}{\mathrm{e}^{k}+\mathrm{e}^{-k}} \mathrm{e}^{\mathrm{i} \mu k}\left(1-\mathrm{e}^{\mathrm{i} \delta k}\right) .
\end{aligned}
$$

$k<0$.

Here $\mathrm{dl} \phi_{-}=\mathrm{dl} \varphi_{-}=\mathrm{dlq} q_{-}=0$, and a derivation similar to above yields

$$
\begin{aligned}
& \mathrm{dlb}=\mathrm{dl} \phi_{0}+\frac{1}{\mathrm{e}^{k}+\mathrm{e}^{-k}}\left(-\mathrm{e}^{-k} \mathrm{dl} \phi_{+}+\mathrm{dl} \mathrm{Y}+\mathrm{e}^{-k} \mathrm{dlB}-\mathrm{e}^{k} \mathrm{dl} \overline{\mathrm{B}}\right), \\
& \mathrm{dly}=\mathrm{dl} \varphi_{0}+\frac{1}{\mathrm{e}^{k}+\mathrm{e}^{-k}}\left(-\mathrm{e}^{-k} \mathrm{dl} \varphi_{+}+\mathrm{dlB}+\mathrm{dl} \overline{\mathrm{B}}\right),
\end{aligned}
$$

with

$$
\begin{aligned}
\mathrm{dl} \phi_{0}-\frac{\mathrm{e}^{-k}}{\mathrm{e}^{k}+\mathrm{e}^{-k}} \mathrm{dl} \phi_{+} & =-\mathrm{i} \frac{N}{2} \frac{\mathrm{e}^{u k}-\mathrm{e}^{-u k}}{\mathrm{e}^{k}+\mathrm{e}^{-k}}, \\
\mathrm{dl} \varphi_{0}-\frac{\mathrm{e}^{-k}}{\mathrm{e}^{k}+\mathrm{e}^{-k}} \mathrm{dl} \varphi_{+} & =-\mathrm{i} \frac{1}{\mathrm{e}^{k}+\mathrm{e}^{-k}} \mathrm{e}^{\mathrm{i} \mu k}\left(1-\mathrm{e}^{\mathrm{i} \delta k}\right) .
\end{aligned}
$$

These results and an expression for dlb similar to that for dlb are Fourier transformed, yielding integral equations for the derivatives of the logarithms of the auxiliary functions $\mathfrak{b}, \overline{\mathfrak{b}}$ and $y$ in terms of $\mathfrak{B}, \overline{\mathfrak{B}}$ and $Y$. Integrating these equations and fixing the integration constants from the known asymptotics completes our derivation of the NLIE.

We are interested in $\Lambda_{1}$ for which we derive from (B.2d)

$$
\mathrm{d} l \Lambda_{1}=\frac{1}{\mathrm{e}^{k}+\mathrm{e}^{-k}}\left(\mathrm{dl} \mathrm{Y}+\mathrm{e}^{-3|k|} \mathrm{dl} \phi_{\mp}+\mathrm{e}^{-2|k|} \mathrm{dl} \varphi_{\mp}\right) .
$$




\section{Appendix C: A simple nearest neighbour correlator}

We are going to relate the derivative of the eigenvalue $\left.\frac{\partial}{\partial \delta} \ln \Lambda^{[1]}(\lambda)\right|_{\delta=0}$ of the spin-1/2 column-to-column transfer matrix $t^{[1]}(\lambda ; \mu)$ with spectral parameter $\lambda$ of the spin-1 system modified by two additional horizontal spin- $1 / 2$ lines with spectral parameters $\mu+\mathrm{i}$ and $\mu-\mathrm{i}+\delta$ to a matrix element of the density operator $D^{[1]}(\lambda, \mu)$ of the unmodified spin-1 system for two neighbouring spin-1/2 spaces with spectral parameters $\lambda, \mu$.

We follow [1]. Be $\langle\Psi|$ and $|\Psi\rangle$ normalized left and right eigenstates of $t^{[1]}(\lambda ; \mu)$ with eigenvalue $\Lambda^{[1]}(x ; \mu)$. We then have

$$
\left.\frac{\partial}{\partial \delta} \ln \left\{\Lambda^{[1]}(\lambda ; \mu)\right\}\right|_{\delta=0}=\left.\frac{\partial}{\partial \delta} \ln \left\{\left\langle\Psi\left|t^{[1]}(\lambda ; \mu)\right| \Psi\right\rangle\right\}\right|_{\delta=0}=\left.\frac{\left\langle\Psi\left|\frac{\partial}{\partial \delta} t^{[1]}(\lambda ; \mu)\right| \Psi\right\rangle}{\left\langle\Psi\left|t^{[1]}(\lambda ; \mu)\right| \Psi\right\rangle}\right|_{\delta=0}
$$

where for the last equality we used that $\langle\Psi \mid \Psi\rangle=1$ for all $\delta$. Next we use the factorization of $\langle\Psi|$ and $|\Psi\rangle$ at $\delta=0$ into eigenstates of the original spin-1 system (without the pair of additional horizontal spin-1/2 lines) times a singlet of two spin-1/2 objects. By use of the Yang-Baxter algebra and unitarity we find

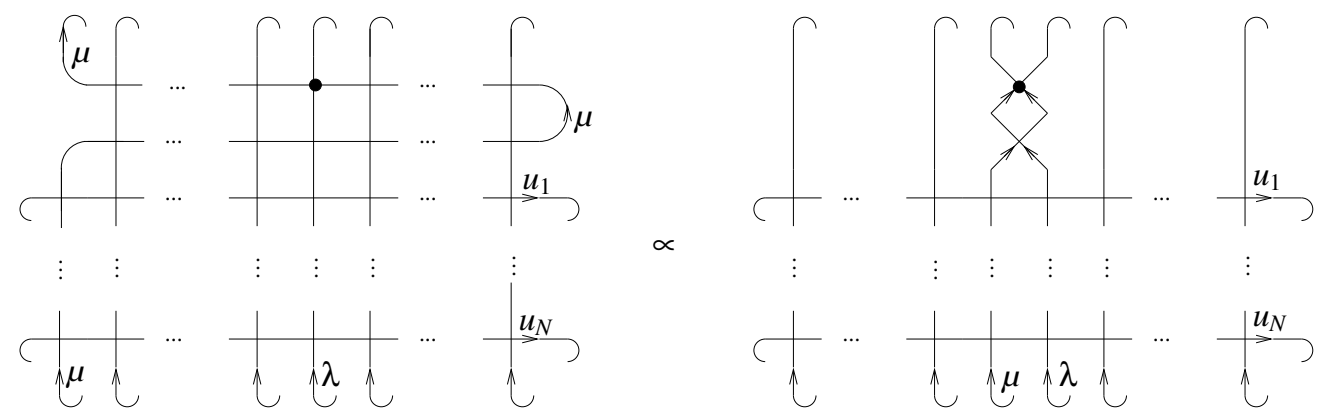

Figure 5: Graphical illustration of the last equation in (C.1).

$$
\left.\frac{\partial}{\partial \delta} \ln \left\{\Lambda^{[1]}(\lambda ; \mu)\right\}\right|_{\delta=0}=\frac{\operatorname{tr}\left\{\left.D^{[1]}(\lambda, \mu) R(\lambda-\mathrm{i}, \mu-\mathrm{i}) \frac{\partial}{\partial \delta} R(\mu+\delta-\mathrm{i}, \lambda-\mathrm{i})\right|_{\delta=0}\right\}}{\operatorname{tr}\left\{D^{[1]}(\lambda, \mu) R(\lambda-\mathrm{i}, \mu-\mathrm{i}) R(\mu-\mathrm{i}, \lambda-\mathrm{i})\right\}} .
$$

Using the normalization of the $\mathcal{R}$ matrices used in the definition of $t^{[1]}(\lambda ; \mu)$ we find unitarity in the following form

$$
\left.R(\lambda-\mathrm{i}, \mu-\mathrm{i}) R(\mu+\delta-\mathrm{i}, \lambda-\mathrm{i})\right|_{\delta=0}=\mathbb{1}
$$

and the derivative

$$
\left.R(\lambda-\mathrm{i}, \mu-\mathrm{i}) \frac{\partial}{\partial \delta} R(\mu+\delta-\mathrm{i}, \lambda-\mathrm{i})\right|_{\delta=0}=\frac{2 \mathrm{i}}{\left.(\lambda-\mu)^{2}+4\right)}\left[\mathbb{1}-P^{[1]}\right] .
$$


Upon introducing the function $\Omega(\lambda, \mu)$ by

$$
\Omega(\lambda, \mu):=\frac{2 \mathrm{i}}{\left.(\lambda-\mu)^{2}+4\right)} \operatorname{tr}\left\{D^{[1]}(\lambda, \mu) P^{[1]}\right\}
$$

we find (63) and the equivalent (62).

\section{Appendix D: More on 3-site density matrix}

In Section 6, the coefficients $\widetilde{\rho}_{j}$ were introduced and only the explicit form of $\widetilde{\rho}_{1}$ was given. Here we comment on the other ones.

Although their explicit forms in terms of $\omega$ are very involved as already commented, the expressions in terms of particular 6-site density matrix elements of spin $\frac{1}{2}$ chain are still manageable.

Let us define three elements

$$
P_{6}=\left(D^{[1]}\right)_{+++++++}^{+++++}, \quad F_{6}=\left(D^{[1]}\right)_{-+++++}^{+++++}, \quad G_{6}=\left(D^{[1]}\right)_{--++++}^{++++--},
$$

where the spin-1/2 density matrix $D^{[1]}$ depends on the six arguments $\left(\xi_{1}^{+}, \xi_{1}^{-}, \xi_{2}^{+}, \xi_{2}^{-}, \xi_{3}^{+}, \xi_{3}^{-}\right)$ where $\xi_{j}^{ \pm}=\xi_{j} \pm \mathrm{i}$ for $j=1,2,3$. Then we find explicitly

$$
\begin{aligned}
& \widetilde{\rho}_{1}\left(\xi_{1}, \xi_{2}, \xi_{3}\right)=\frac{1}{27} \mathcal{N}\left(\xi_{1}, \xi_{2}, \xi_{3}\right)+\frac{14}{9} G_{5}\left(-\xi_{1}^{-},-\xi_{2}^{-},-\xi_{2}^{+},-\xi_{3}^{-},-\xi_{3}^{+}\right) \\
& +\frac{14\left(2 \mathrm{i}+\xi_{1}-\xi_{2}\right)}{9\left(\xi_{1}-\xi_{2}\right)} G_{6}\left(-\xi_{1}^{+},-\xi_{2}^{-},-\xi_{1}^{-},-\xi_{2}^{+},-\xi_{3}^{-},-\xi_{3}^{+}\right)+\frac{2\left(-4 \mathrm{i}+\xi_{2}-\xi_{3}\right)}{3\left(-2 \mathrm{i}+\xi_{2}-\xi_{3}\right)} G_{6}\left(-\xi_{1}^{-},-\xi_{1}^{+},-\xi_{2}^{+},-\xi_{3}^{+},-\xi_{2}^{-},-\xi_{3}^{-}\right) \\
& -2\left(56 \mathrm{i}-28 \xi_{1}-8 \mathrm{i} \xi_{1}^{2}-4 \mathrm{i} \xi_{1} \xi_{2}+\xi_{1}^{2} \xi_{2}+12 \mathrm{i} \xi_{2}^{2}-2 \xi_{1} \xi_{2}^{2}+\xi_{2}^{3}+28 \xi_{3}+20 i \xi_{1} \xi_{3}-\xi_{1}^{2} \xi_{3}\right. \\
& \left.-20 i \xi_{2} \xi_{3}+2 \xi_{1} \xi_{2} \xi_{3}-\xi_{2}^{2} \xi_{3}\right) \times \frac{G_{6}\left(-\xi_{1}^{-},-\xi_{1}^{+},-\xi_{2}^{-},-\xi_{2}^{+},-\xi_{3}^{-},-\xi_{3}^{+}\right)}{9\left(\xi_{1}-\xi_{2}\right)\left(2 i+\xi_{1}-\xi_{2}\right)\left(-2 i+\xi_{2}-\xi_{3}\right)} \\
& -\frac{4\left(4 \mathrm{i}+\xi_{1}-\xi_{2}\right)}{2 i+\xi_{1}-\xi_{2}} G_{6}\left(-\xi_{1}^{-},-\xi_{2}^{-},-\xi_{1}^{+},-\xi_{2}^{+},-\xi_{3}^{-},-\xi_{3}^{+}\right)-\frac{2\left(4 \mathrm{i}+\xi_{2}-\xi_{3}\right)\left(-6 \mathrm{i}+\xi_{2}-\xi_{3}\right)}{3\left(-2 \mathrm{i}+\xi_{2}-\xi_{3}\right)\left(2 \mathrm{i}+\xi_{2}-\xi_{3}\right)} G_{6}\left(\xi_{1}^{-}, \xi_{1}^{+}, \xi_{2}^{-}, \xi_{3}^{-}, \xi_{2}^{+}, \xi_{3}^{+}\right) \\
& -\frac{\left(2 \mathrm{i}+\xi_{2}-\xi_{3}\right)\left(4 \mathrm{i}+\xi_{2}-\xi_{3}\right)}{3\left(\xi_{2}-\xi_{3}\right)\left(-2 \mathrm{i}+\xi_{2}-\xi_{3}\right)} G_{6}\left(\xi_{1}^{-}, \xi_{1}^{+}, \xi_{3}^{-}, \xi_{3}^{+}, \xi_{2}^{-}, \xi_{2}^{+}\right)-\frac{2\left(-4 \mathrm{i}+\xi_{1}-\xi_{2}\right)\left(6 \mathrm{i}+\xi_{1}-\xi_{2}\right)}{3\left(-2 \mathrm{i}+\xi_{1}-\xi_{2}\right)\left(2 \mathrm{i}+\xi_{1}-\xi_{2}\right)} G_{6}\left(\xi_{1}^{+}, \xi_{2}^{+}, \xi_{1}^{-}, \xi_{2}^{-}, \xi_{3}^{-}, \xi_{3}^{+}\right) \\
& -\frac{\left(-2 \mathrm{i}+\xi_{1}-\xi_{2}\right)\left(-4 \mathrm{i}+\xi_{1}-\xi_{2}\right)}{3\left(\xi_{1}-\xi_{2}\right)\left(2 \mathrm{i}+\xi_{1}-\xi_{2}\right)} G_{6}\left(\xi_{2}^{-}, \xi_{2}^{+}, \xi_{1}^{-}, \xi_{1}^{+}, \xi_{3}^{-}, \xi_{3}^{+}\right) \\
& -\left(48 i \xi_{1}-24 \xi_{1}^{2}-96 i \xi_{2}+4 \xi_{1} \xi_{2}-10 i \xi_{1}^{2} \xi_{2}-4 \xi_{2}^{2}+30 i \xi_{1} \xi_{2}^{2}+\xi_{1}^{2} \xi_{2}^{2}-20 i \xi_{2}^{3}-2 \xi_{1} \xi_{2}^{3}+\xi_{2}^{4}+48 i \xi_{3}\right. \\
& +44 \xi_{1} \xi_{3}+10 i \xi_{1}^{2} \xi_{3}+4 \xi_{2} \xi_{3}-40 i \xi_{1} \xi_{2} \xi_{3}-2 \xi_{1}^{2} \xi_{2} \xi_{3}+30 i \xi_{2}^{2} \xi_{3}+4 \xi_{1} \xi_{2}^{2} \xi_{3}-2 \xi_{2}^{3} \xi_{3}-24 \xi_{3}^{2}+10 i \xi_{1} \xi_{3}^{2} \\
& \left.+\xi_{1}^{2} \xi_{3}^{2}-10 \mathrm{i} \xi_{2} \xi_{3}^{2}-2 \xi_{1} \xi_{2} \xi_{3}^{2}+\xi_{2}^{2} \xi_{3}^{2}\right) \frac{G_{6}\left(\xi_{1}^{-}, \xi_{1}^{+}, \xi_{2}^{-}, \xi_{2}^{+}, \xi_{3}^{-}, \xi_{3}^{+}\right)}{9\left(\xi_{1}-\xi_{2}\right)\left(-2 \mathrm{i}+\xi_{1}-\xi_{2}\right)\left(\xi_{2}-\xi_{3}\right)\left(2 \mathrm{i}+\xi_{2}-\xi_{3}\right)}
\end{aligned}
$$




$$
\begin{aligned}
& \widetilde{\rho}_{2}\left(\xi_{1}, \xi_{2}, \xi_{3}\right)=-\frac{1}{27} \mathcal{N}\left(\xi_{1}, \xi_{2}, \xi_{3}\right)-\frac{14}{9} G_{5}\left(-\xi_{1}^{-},-\xi_{2}^{-},-\xi_{2}^{+},-\xi_{3}^{-},-\xi_{3}^{+}\right) \\
& -\frac{2\left(4 \mathrm{i}+\xi_{1}-\xi_{2}\right)}{\xi_{1}-\xi_{2}} F_{6}\left(\xi_{1}^{+}, \xi_{1}^{-}, \xi_{2}^{-}, \xi_{2}^{+}, \xi_{3}^{-}, \xi_{3}^{+}\right)-\frac{2\left(-4 \mathrm{i}+\xi_{1}-\xi_{2}\right)}{\xi_{1}-\xi_{2}} F_{6}\left(\xi_{2}^{+}, \xi_{1}^{-}, \xi_{1}^{+}, \xi_{2}^{-}, \xi_{3}^{-}, \xi_{3}^{+}\right) \\
& -\frac{14\left(2 \mathrm{i}+\xi_{1}-\xi_{2}\right)}{9\left(\xi_{1}-\xi_{2}\right)} G_{6}\left(-\xi_{1}^{+},-\xi_{2}^{-},-\xi_{1}^{-},-\xi_{2}^{+},-\xi_{3}^{-},-\xi_{3}^{+}\right)-\frac{2\left(-4 i+\xi_{2}-\xi_{3}\right)}{3\left(-2 \mathrm{i}+\xi_{2}-\xi_{3}\right)} G_{6}\left(-\xi_{1}^{-},-\xi_{1}^{+},-\xi_{2}^{+},-\xi_{3}^{+},-\xi_{2}^{-},-\xi_{3}^{-}\right) \\
& +2\left(56 \mathrm{i}-28 \xi_{1}-8 \mathrm{i} \xi_{1}^{2}-4 \mathrm{i} \xi_{1} \xi_{2}+\xi_{1}^{2} \xi_{2}+12 \mathrm{i} \xi_{2}^{2}-2 \xi_{1} \xi_{2}^{2}+\xi_{2}^{3}+28 \xi_{3}+20 i \xi_{1} \xi_{3}-\xi_{1}^{2} \xi_{3}-20 i \xi_{2} \xi_{3}\right. \\
& \left.+2 \xi_{1} \xi_{2} \xi_{3}-\xi_{2}^{2} \xi_{3}\right) \frac{G_{6}\left(-\xi_{1}^{-},-\xi_{1}^{+},-\xi_{2}^{-},-\xi_{2}^{+},-\xi_{3}^{-},-\xi_{3}^{+}\right)}{9\left(\xi_{1}-\xi_{2}\right)\left(2 i+\xi_{1}-\xi_{2}\right)\left(-2 i+\xi_{2}-\xi_{3}\right)} \\
& +\frac{4\left(4 i+\xi_{1}-\xi_{2}\right)}{2 i+\xi_{1}-\xi_{2}} G_{6}\left(-\xi_{1}^{-},-\xi_{2}^{-},-\xi_{1}^{+},-\xi_{2}^{+},-\xi_{3}^{-},-\xi_{3}^{+}\right) \\
& +\left(48 i \xi_{1}-24 \xi_{1}^{2}-96 i \xi_{2}+4 \xi_{1} \xi_{2}-10 i \xi_{1}^{2} \xi_{2}-4 \xi_{2}^{2}+30 i \xi_{1} \xi_{2}^{2}+\xi_{1}^{2} \xi_{2}^{2}-20 i \xi_{2}^{3}-2 \xi_{1} \xi_{2}^{3}+\xi_{2}^{4}\right. \\
& +48 i \xi_{3}+44 \xi_{1} \xi_{3}+10 i \xi_{1}^{2} \xi_{3}+4 \xi_{2} \xi_{3}-40 i \xi_{1} \xi_{2} \xi_{3}-2 \xi_{1}^{2} \xi_{2} \xi_{3}+30 i \xi_{2}^{2} \xi_{3}+4 \xi_{1} \xi_{2}^{2} \xi_{3}-2 \xi_{2}^{3} \xi_{3} \\
& \left.-24 \xi_{3}^{2}+10 i \xi_{1} \xi_{3}^{2}+\xi_{1}^{2} \xi_{3}^{2}-10 i \xi_{2} \xi_{3}^{2}-2 \xi_{1} \xi_{2} \xi_{3}^{2}+\xi_{2}^{2} \xi_{3}^{2}\right) \times \frac{G_{6}\left(\xi_{1}^{-}, \xi_{1}^{+}, \xi_{2}^{-}, \xi_{2}^{+}, \xi_{3}^{-}, \xi_{3}^{+}\right)}{9\left(\xi_{1}-\xi_{2}\right)\left(-2 i+\xi_{1}-\xi_{2}\right)\left(\xi_{2}-\xi_{3}\right)\left(2 i+\xi_{2}-\xi_{3}\right)} \\
& +\frac{2\left(4 \mathrm{i}+\xi_{2}-\xi_{3}\right)\left(-6 \mathrm{i}+\xi_{2}-\xi_{3}\right)}{3\left(-2 \mathrm{i}+\xi_{2}-\xi_{3}\right)\left(2 \mathrm{i}+\xi_{2}-\xi_{3}\right)} G_{6}\left(\xi_{1}^{-}, \xi_{1}^{+}, \xi_{2}^{-}, \xi_{3}^{-}, \xi_{2}^{+}, \xi_{3}^{+}\right)+\frac{\left(2 \mathrm{i}+\xi_{2}-\xi_{3}\right)\left(4 \mathrm{i}+\xi_{2}-\xi_{3}\right)}{3\left(\xi_{2}-\xi_{3}\right)\left(-2 \mathrm{i}+\xi_{2}-\xi_{3}\right)} G_{6}\left(\xi_{1}^{-}, \xi_{1}^{+}, \xi_{3}^{-}, \xi_{3}^{+}, \xi_{2}^{-}, \xi_{2}^{+}\right) \\
& +\frac{2\left(-4 \mathrm{i}+\xi_{1}-\xi_{2}\right)\left(6 \mathrm{i}+\xi_{1}-\xi_{2}\right)}{3\left(-2 \mathrm{i}+\xi_{1}-\xi_{2}\right)\left(2 \mathrm{i}+\xi_{1}-\xi_{2}\right)} G_{6}\left(\xi_{1}^{+}, \xi_{2}^{+}, \xi_{1}^{-}, \xi_{2}^{-}, \xi_{3}^{-}, \xi_{3}^{+}\right)+\frac{\left(-2 \mathrm{i}+\xi_{1}-\xi_{2}\right)\left(-4 \mathrm{i}+\xi_{1}-\xi_{2}\right)}{3\left(\xi_{1}-\xi_{2}\right)\left(2 \mathrm{i}+\xi_{1}-\xi_{2}\right)} G_{6}\left(\xi_{2}^{-}, \xi_{2}^{+}, \xi_{1}^{-}, \xi_{1}^{+}, \xi_{3}^{-}, \xi_{3}^{+}\right) \\
& +P_{6}\left(\xi_{1}^{-}, \xi_{1}^{+}, \xi_{2}^{-}, \xi_{2}^{+}, \xi_{3}^{-}, \xi_{3}^{+}\right) \\
& \widetilde{\rho}_{4}\left(\xi_{1}, \xi_{2}, \xi_{3}\right)=\frac{1}{27} \mathcal{N}\left(\xi_{1}, \xi_{2}, \xi_{3}\right)+\frac{14}{9} G_{5}\left(-\xi_{1}^{-},-\xi_{2}^{-},-\xi_{2}^{+},-\xi_{3}^{-},-\xi_{3}^{+}\right)-2 F_{6}\left(-\xi_{1}^{-},-\xi_{1}^{+},-\xi_{2}^{-},-\xi_{2}^{+},-\xi_{3}^{+},-\xi_{3}^{-}\right) \\
& +\frac{4\left(-2 \mathrm{i} \xi_{1}+4 \mathrm{i} \xi_{2}+\xi_{1} \xi_{2}-\xi_{2}^{2}-2 \mathrm{i} \xi_{3}-\xi_{1} \xi_{3}+\xi_{2} \xi_{3}\right)}{\left(\xi_{1}-\xi_{2}\right)\left(\xi_{2}-\xi_{3}\right)} F_{6}\left(\xi_{1}^{+}, \xi_{1}^{-}, \xi_{2}^{-}, \xi_{2}^{+}, \xi_{3}^{-}, \xi_{3}^{+}\right) \\
& +\frac{2\left(4 i+\xi_{2}-\xi_{3}\right)}{\xi_{2}-\xi_{3}} F_{6}\left(\xi_{1}^{+}, \xi_{1}^{-}, \xi_{2}^{-}, \xi_{3}^{-}, \xi_{3}^{+}, \xi_{2}^{+}\right)+\frac{2\left(-4 i+\xi_{1}-\xi_{2}\right)}{\xi_{1}-\xi_{2}} F_{6}\left(\xi_{2}^{+}, \xi_{1}^{-}, \xi_{1}^{+}, \xi_{2}^{-}, \xi_{3}^{-}, \xi_{3}^{+}\right) \\
& +\frac{14\left(2 \mathrm{i}+\xi_{1}-\xi_{2}\right) G_{6}\left(-\xi_{1}^{+},-\xi_{2}^{-},-\xi_{1}^{-},-\xi_{2}^{+},-\xi_{3}^{-},-\xi_{3}^{+}\right)}{9\left(\xi_{1}-\xi_{2}\right)}+\frac{2\left(-4 i+\xi_{2}-\xi_{3}\right) G_{6}\left(-\xi_{1}^{-},-\xi_{1}^{+},-\xi_{2}^{+},-\xi_{3}^{+},-\xi_{2}^{-},-\xi_{3}^{-}\right)}{3\left(-2 \mathrm{i}+\xi_{2}-\xi_{3}\right)} \\
& -2\left(56 \mathrm{i}-28 \xi_{1}-8 \mathrm{i} \xi_{1}^{2}-4 \mathrm{i} \xi_{1} \xi_{2}+\xi_{1}^{2} \xi_{2}+12 i \xi_{2}^{2}-2 \xi_{1} \xi_{2}^{2}+\xi_{2}^{3}+28 \xi_{3}+20 i \xi_{1} \xi_{3}-\xi_{1}^{2} \xi_{3}\right. \\
& \left.-20 \mathrm{i} \xi_{2} \xi_{3}+2 \xi_{1} \xi_{2} \xi_{3}-\xi_{2}^{2} \xi_{3}\right) \times \frac{G_{6}\left(-\xi_{1}^{-},-\xi_{1}^{+},-\xi_{2}^{-},-\xi_{2}^{+},-\xi_{3}^{-},-\xi_{3}^{+}\right)}{9\left(\xi_{1}-\xi_{2}\right)\left(2 \mathrm{i}+\xi_{1}-\xi_{2}\right)\left(-2 \mathrm{i}+\xi_{2}-\xi_{3}\right)} \\
& -\left(48 i \xi_{1}-24 \xi_{1}^{2}-96 i \xi_{2}+4 \xi_{1} \xi_{2}-10 i \xi_{1}^{2} \xi_{2}-4 \xi_{2}^{2}+30 i \xi_{1} \xi_{2}^{2}+\xi_{1}^{2} \xi_{2}^{2}-20 i \xi_{2}^{3}-2 \xi_{1} \xi_{2}^{3}+\xi_{2}^{4}\right. \\
& +48 i \xi_{3}+44 \xi_{1} \xi_{3}+10 i \xi_{1}^{2} \xi_{3}+4 \xi_{2} \xi_{3}-40 i \xi_{1} \xi_{2} \xi_{3}-2 \xi_{1}^{2} \xi_{2} \xi_{3}+30 i \xi_{2}^{2} \xi_{3}+4 \xi_{1} \xi_{2}^{2} \xi_{3}-2 \xi_{2}^{3} \xi_{3}-24 \xi_{3}^{2} \\
& \left.+10 i \xi_{1} \xi_{3}^{2}+\xi_{1}^{2} \xi_{3}^{2}-10 i \xi_{2} \xi_{3}^{2}-2 \xi_{1} \xi_{2} \xi_{3}^{2}+\xi_{2}^{2} \xi_{3}^{2}\right) \times \frac{G_{6}\left(\xi_{1}^{-}, \xi_{1}^{+}, \xi_{2}^{-}, \xi_{2}^{+}, \xi_{3}^{-}, \xi_{3}^{+}\right)}{9\left(\xi_{1}-\xi_{2}\right)\left(-2 i+\xi_{1}-\xi_{2}\right)\left(\xi_{2}-\xi_{3}\right)\left(2 i+\xi_{2}-\xi_{3}\right)} \\
& -\frac{2\left(4 \mathrm{i}+\xi_{2}-\xi_{3}\right)\left(-6 \mathrm{i}+\xi_{2}-\xi_{3}\right)}{3\left(-2 \mathrm{i}+\xi_{2}-\xi_{3}\right)\left(2 \mathrm{i}+\xi_{2}-\xi_{3}\right)} G_{6}\left(\xi_{1}^{-}, \xi_{1}^{+}, \xi_{2}^{-}, \xi_{3}^{-}, \xi_{2}^{+}, \xi_{3}^{+}\right)-\frac{4\left(4 \mathrm{i}+\xi_{1}-\xi_{2}\right)}{2 i+\xi_{1}-\xi_{2}} G_{6}\left(-\xi_{1}^{-},-\xi_{2}^{-},-\xi_{1}^{+},-\xi_{2}^{+},-\xi_{3}^{-},-\xi_{3}^{+}\right) \\
& -\frac{\left(2 \mathrm{i}+\xi_{2}-\xi_{3}\right)\left(4 \mathrm{i}+\xi_{2}-\xi_{3}\right)}{3\left(\xi_{2}-\xi_{3}\right)\left(-2 \mathrm{i}+\xi_{2}-\xi_{3}\right)} G_{6}\left(\xi_{1}^{-}, \xi_{1}^{+}, \xi_{3}^{-}, \xi_{3}^{+}, \xi_{2}^{-}, \xi_{2}^{+}\right)-\frac{2\left(-4 \mathrm{i}+\xi_{1}-\xi_{2}\right)\left(6 \mathrm{i}+\xi_{1}-\xi_{2}\right)}{3\left(-2 \mathrm{i}+\xi_{1}-\xi_{2}\right)\left(2 \mathrm{i}+\xi_{1}-\xi_{2}\right)} G_{6}\left(\xi_{1}^{+}, \xi_{2}^{+}, \xi_{1}^{-}, \xi_{2}^{-}, \xi_{3}^{-}, \xi_{3}^{+}\right) \\
& -\frac{\left(-2 \mathrm{i}+\xi_{1}-\xi_{2}\right)\left(-4 \mathrm{i}+\xi_{1}-\xi_{2}\right)}{3\left(\xi_{1}-\xi_{2}\right)\left(2 \mathrm{i}+\xi_{1}-\xi_{2}\right)} G_{6}\left(\xi_{2}^{-}, \xi_{2}^{+}, \xi_{1}^{-}, \xi_{1}^{+}, \xi_{3}^{-}, \xi_{3}^{+}\right)-P_{6}\left(\xi_{1}^{-}, \xi_{1}^{+}, \xi_{2}^{-}, \xi_{2}^{+}, \xi_{3}^{-}, \xi_{3}^{+}\right)
\end{aligned}
$$




$$
\begin{aligned}
& \tilde{\rho}_{6}\left(\xi_{1}, \xi_{2}, \xi_{3}\right)=-\frac{1}{27} \mathcal{N}\left(\xi_{1}, \xi_{2}, \xi_{3}\right)-\frac{14}{9} G_{5}\left(-\xi_{1}^{-},-\xi_{2}^{-},-\xi_{2}^{+},-\xi_{3}^{-},-\xi_{3}^{+}\right)+2 F_{6}\left(-\xi_{1}^{-},-\xi_{1}^{+},-\xi_{2}^{-},-i-\xi_{2},-\xi_{3}^{+},-\xi_{3}^{-}\right) \\
& -\frac{2\left(-4 i \xi_{1}+8 i \xi_{2}+\xi_{1} \xi_{2}-\xi_{2}^{2}-4 i \xi_{3}-\xi_{1} \xi_{3}+\xi_{2} \xi_{3}\right)}{\left(\xi_{1}-\xi_{2}\right)\left(\xi_{2}-\xi_{3}\right)} F_{6}\left(\xi_{1}^{+}, \xi_{1}^{-}, \xi_{2}^{-}, \xi_{2}^{+}, \xi_{3}^{-}, \xi_{3}^{+}\right) \\
& -\frac{2\left(4 \mathrm{i}+\xi_{2}-\xi_{3}\right) F_{6}\left(\xi_{1}^{+}, \xi_{1}^{-}, \xi_{2}^{-}, \xi_{3}^{-}, \xi_{3}^{+}, \xi_{2}^{+}\right)}{\xi_{2}-\xi_{3}}-\frac{2\left(-4 \mathrm{i}+\xi_{1}-\xi_{2}\right) F_{6}\left(\xi_{2}^{+}, \xi_{1}^{-}, \xi_{1}^{+}, \xi_{2}^{-}, \xi_{3}^{-}, \xi_{3}^{+}\right)}{\xi_{1}-\xi_{2}} \\
& -\frac{14\left(2 \mathrm{i}+\xi_{1}-\xi_{2}\right) G_{6}\left(-\xi_{1}^{+},-\xi_{2}^{-},-\xi_{1}^{-},-\xi_{2}^{+},-\xi_{3}^{-},-\xi_{3}^{+}\right)}{9\left(\xi_{1}-\xi_{2}\right)}-\frac{2\left(-4 i+\xi_{2}-\xi_{3}\right) G_{6}\left(-\xi_{1}^{-},-\xi_{1}^{+},-\xi_{2}^{+},-\xi_{3}^{+},-\xi_{2}^{-},-\xi_{3}^{-}\right)}{3\left(-2 \mathrm{i}+\xi_{2}-\xi_{3}\right)} \\
& +2\left(56 \mathrm{i}-28 \xi_{1}-8 \mathrm{i} \xi_{1}^{2}-4 \mathrm{i} \xi_{1} \xi_{2}+\xi_{1}^{2} \xi_{2}+12 i \xi_{2}^{2}-2 \xi_{1} \xi_{2}^{2}+\xi_{2}^{3}+28 \xi_{3}+20 i \xi_{1} \xi_{3}-\xi_{1}^{2} \xi_{3}\right. \\
& \left.20 \mathrm{i} \xi_{2} \xi_{3}+2 \xi_{1} \xi_{2} \xi_{3}-\xi_{2}^{2} \xi_{3}\right) \times \frac{G_{6}\left(-\xi_{1}^{-},-\xi_{1}^{+},-\xi_{2}^{-},-\xi_{2}^{+},-\xi_{3}^{-},-\xi_{3}^{+}\right)}{9\left(\xi_{1}-\xi_{2}\right)\left(2 \mathrm{i}+\xi_{1}-\xi_{2}\right)\left(-2 \mathrm{i}+\xi_{2}-\xi_{3}\right)} \\
& +\left(48 i \xi_{1}-24 \xi_{1}^{2}-96 i \xi_{2}+4 \xi_{1} \xi_{2}-10 i \xi_{1}^{2} \xi_{2}-4 \xi_{2}^{2}+30 i \xi_{1} \xi_{2}^{2}+\xi_{1}^{2} \xi_{2}^{2}-20 i \xi_{2}^{3}-2 \xi_{1} \xi_{2}^{3}+\xi_{2}^{4}+48 i \xi_{3}\right. \\
& +44 \xi_{1} \xi_{3}+10 i \xi_{1}^{2} \xi_{3}+4 \xi_{2} \xi_{3}-40 i \xi_{1} \xi_{2} \xi_{3}-2 \xi_{1}^{2} \xi_{2} \xi_{3}+30 i \xi_{2}^{2} \xi_{3}+4 \xi_{1} \xi_{2}^{2} \xi_{3}-2 \xi_{2}^{3} \xi_{3}-24 \xi_{3}^{2}
\end{aligned}
$$

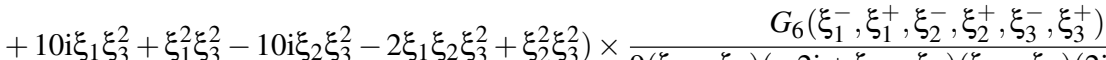

$$
\begin{aligned}
& +\frac{4\left(4 \mathrm{i}+\xi_{1}-\xi_{2}\right) G_{6}\left(-\xi_{1}^{-},-\xi_{2}^{-},-\xi_{1}^{+},-\xi_{2}^{+},-\xi_{3}^{-},-\xi_{3}^{+}\right)}{2 i+\xi_{1}-\xi_{2}}+\frac{2\left(4 \mathrm{i}+\xi_{2}-\xi_{3}\right)\left(-6 \mathrm{i}+\xi_{2}-\xi_{3}\right)}{3\left(-2 \mathrm{i}+\xi_{2}-\xi_{3}\right)\left(2 \mathrm{i}+\xi_{2}-\xi_{3}\right)} G_{6}\left(\xi_{1}^{-}, \xi_{1}^{+}, \xi_{2}^{-}, \xi_{3}^{-}, \xi_{2}^{+}, \xi_{3}^{+}\right) \\
& +\frac{\left(2 \mathrm{i}+\xi_{2}-\xi_{3}\right)\left(4 \mathrm{i}+\xi_{2}-\xi_{3}\right)}{3\left(\xi_{2}-\xi_{3}\right)\left(-2 \mathrm{i}+\xi_{2}-\xi_{3}\right)} G_{6}\left(\xi_{1}^{-}, \xi_{1}^{+}, \xi_{3}^{-}, \xi_{3}^{+}, \xi_{2}^{-}, \xi_{2}^{+}\right)+\frac{2\left(-4 \mathrm{i}+\xi_{1}-\xi_{2}\right)\left(6 \mathrm{i}+\xi_{1}-\xi_{2}\right)}{3\left(-2 \mathrm{i}+\xi_{1}-\xi_{2}\right)\left(2 \mathrm{i}+\xi_{1}-\xi_{2}\right)} G_{6}\left(\xi_{1}^{+}, \xi_{2}^{+}, \xi_{1}^{-}, \xi_{2}^{-}, \xi_{3}^{-}, \xi_{3}^{+}\right) \\
& +\frac{\left(-2 \mathrm{i}+\xi_{1}-\xi_{2}\right)\left(-4 \mathrm{i}+\xi_{1}-\xi_{2}\right)}{3\left(\xi_{1}-\xi_{2}\right)\left(2 \mathrm{i}+\xi_{1}-\xi_{2}\right)} G_{6}\left(\xi_{2}^{-}, \xi_{2}^{+}, \xi_{1}^{-}, \xi_{1}^{+}, \xi_{3}^{-}, \xi_{3}^{+}\right)+P_{6}\left(\xi_{1}^{-}, \xi_{1}^{+}, \xi_{2}^{-}, \xi_{2}^{+}, \xi_{3}^{-}, \xi_{3}^{+}\right) \\
& \widetilde{\rho}_{7}\left(\xi_{1}, \xi_{2}, \xi_{3}\right)=\frac{1}{27} \mathcal{N}\left(\xi_{1}, \xi_{2}, \xi_{3}\right)-\frac{4}{9} G_{5}\left(-\xi_{1}^{-},-\xi_{2}^{-},-\xi_{2}^{+},-\xi_{3}^{-},-\xi_{3}^{+}\right) \\
& +\frac{2\left(-4 i+\xi_{2}-\xi_{3}\right)}{\xi_{2}-\xi_{3}} F_{6}\left(\xi_{1}^{+}, \xi_{1}^{-}, \xi_{2}^{-}, \xi_{2}^{+}, \xi_{3}^{-}, \xi_{3}^{+}\right)+\frac{2\left(4 i+\xi_{2}-\xi_{3}\right)}{\xi_{2}-\xi_{3}} F_{6}\left(\xi_{1}^{+}, \xi_{1}^{-}, \xi_{2}^{-}, \xi_{3}^{-}, \xi_{3}^{+}, \xi_{2}^{+}\right) \\
& -\frac{4\left(2 \mathrm{i}+\xi_{1}-\xi_{2}\right)}{9\left(\xi_{1}-\xi_{2}\right)} G_{6}\left(-\xi_{1}^{+},-\xi_{2}^{-},-\xi_{1}^{-},-\xi_{2}^{+},-\xi_{3}^{-},-\xi_{3}^{+}\right)+\frac{2\left(-4 i+\xi_{2}-\xi_{3}\right)}{3\left(-2 \mathrm{i}+\xi_{2}-\xi_{3}\right)} G_{6}\left(-\xi_{1}^{-},-\xi_{1}^{+},-\xi_{2}^{+},-\xi_{3}^{+},-\xi_{2}^{-},-\xi_{3}^{-}\right) \\
& -\frac{2\left(-8-8 i \xi_{1}+4 i \xi_{2}+\xi_{1} \xi_{2}-\xi_{2}^{2}+4 i \xi_{3}-\xi_{1} \xi_{3}+\xi_{2} \xi_{3}\right)}{9\left(\xi_{1}-\xi_{2}\right)\left(-2 i+\xi_{2}-\xi_{3}\right)} G_{6}\left(-\xi_{1}^{-},-\xi_{1}^{+},-\xi_{2}^{-},-\xi_{2}^{+},-\xi_{3}^{-},-\xi_{3}^{+}\right) \\
& -\left(48 i \xi_{1}-24 \xi_{1}^{2}+48 i \xi_{2}+76 \xi_{1} \xi_{2}-10 i \xi_{1}^{2} \xi_{2}-4 \xi_{2}^{2}-6 i \xi_{1} \xi_{2}^{2}+\xi_{1}^{2} \xi_{2}^{2}+16 i \xi_{2}^{3}-2 \xi_{1} \xi_{2}^{3}+\xi_{2}^{4}-96 i \xi_{3}\right. \\
& -28 \xi_{1} \xi_{3}+10 \mathrm{i} \xi_{1}^{2} \xi_{3}-68 \xi_{2} \xi_{3}+32 \mathrm{i} \xi_{1} \xi_{2} \xi_{3}-2 \xi_{1}^{2} \xi_{2} \xi_{3}-42 \mathrm{i} \xi_{2}^{2} \xi_{3}+4 \xi_{1} \xi_{2}^{2} \xi_{3}-2 \xi_{2}^{3} \xi_{3}+48 \xi_{3}^{2} \\
& \left.-26 i \xi_{1} \xi_{3}^{2}+\xi_{1}^{2} \xi_{3}^{2}+26 i \xi_{2} \xi_{3}^{2}-2 \xi_{1} \xi_{2} \xi_{3}^{2}+\xi_{2}^{2} \xi_{3}^{2}\right) \times \frac{G_{6}\left(\xi_{1}^{-}, \xi_{1}^{+}, \xi_{2}^{-}, \xi_{2}^{+}, \xi_{3}^{-}, \xi_{3}^{+}\right)}{9\left(\xi_{1}-\xi_{2}\right)\left(-2 \mathrm{i}+\xi_{1}-\xi_{2}\right)\left(\xi_{2}-\xi_{3}\right)\left(2 \mathrm{i}+\xi_{2}-\xi_{3}\right)} \\
& -\frac{2\left(4 \mathrm{i}+\xi_{2}-\xi_{3}\right)\left(-6 \mathrm{i}+\xi_{2}-\xi_{3}\right)}{3\left(-2 \mathrm{i}+\xi_{2}-\xi_{3}\right)\left(2 \mathrm{i}+\xi_{2}-\xi_{3}\right)} G_{6}\left(\xi_{1}^{-}, \xi_{1}^{+}, \xi_{2}^{-}, \xi_{3}^{-}, \xi_{2}^{+}, \xi_{3}^{+}\right)-\frac{\left(2 \mathrm{i}+\xi_{2}-\xi_{3}\right)\left(4 \mathrm{i}+\xi_{2}-\xi_{3}\right)}{3\left(\xi_{2}-\xi_{3}\right)\left(-2 \mathrm{i}+\xi_{2}-\xi_{3}\right)} G_{6}\left(\xi_{1}^{-}, \xi_{1}^{+}, \xi_{3}^{-}, \xi_{3}^{+}, \xi_{2}^{-}, \xi_{2}^{+}\right) \\
& +\frac{4\left(-4 \mathrm{i}+\xi_{1}-\xi_{2}\right)\left(6 \mathrm{i}+\xi_{1}-\xi_{2}\right) G_{6}\left(\xi_{1}^{+}, \xi_{2}^{+}, \xi_{1}^{-}, \xi_{2}^{-}, \xi_{3}^{-}, \xi_{3}^{+}\right)}{3\left(-2 \mathrm{i}+\xi_{1}-\xi_{2}\right)\left(2 \mathrm{i}+\xi_{1}-\xi_{2}\right)}+\frac{2\left(-2 \mathrm{i}+\xi_{1}-\xi_{2}\right)\left(-4 \mathrm{i}+\xi_{1}-\xi_{2}\right)}{3\left(\xi_{1}-\xi_{2}\right)\left(2 \mathrm{i}+\xi_{1}-\xi_{2}\right)} G_{6}\left(\xi_{2}^{-}, \xi_{2}^{+}, \xi_{1}^{-}, \xi_{1}^{+}, \xi_{3}^{-}, \xi_{3}^{+}\right) \\
& -P_{6}\left(\xi_{1}^{-}, \xi_{1}^{+}, \xi_{2}^{-}, \xi_{2}^{+}, \xi_{3}^{-}, \xi_{3}^{+}\right)
\end{aligned}
$$




$$
\begin{aligned}
& \widetilde{\rho}_{9}\left(\xi_{1}, \xi_{2}, \xi_{3}\right)=\frac{1}{27} \mathcal{N}\left(\xi_{1}, \xi_{2}, \xi_{3}\right)-\frac{4}{9} G_{5}\left(-\xi_{1}^{-},-\xi_{2}^{-},-\xi_{2}^{+},-\xi_{3}^{-},-\xi_{3}^{+}\right)-2 F_{6}\left(-\xi_{1}^{-},-\xi_{1}^{+},-\xi_{2}^{-},-\xi_{2}^{+},-\xi_{3}^{+},-\xi_{3}^{-}\right) \\
& +\frac{2\left(-4 \mathrm{i} \xi_{1}+8 \mathrm{i} \xi_{2}+\xi_{1} \xi_{2}-\xi_{2}^{2}-4 \mathrm{i} \xi_{3}-\xi_{1} \xi_{3}+\xi_{2} \xi_{3}\right)}{\left(\xi_{1}-\xi_{2}\right)\left(\xi_{2}-\xi_{3}\right)} F_{6}\left(\xi_{1}^{+}, \xi_{1}^{-}, \xi_{2}^{-}, \xi_{2}^{+}, \xi_{3}^{-}, \xi_{3}^{+}\right) \\
& +\frac{2\left(4 \mathrm{i}+\xi_{2}-\xi_{3}\right)}{\xi_{2}-\xi_{3}} F_{6}\left(\xi_{1}^{+}, \xi_{1}^{-}, \xi_{2}^{-}, \xi_{3}^{-}, \xi_{3}^{+}, \xi_{2}^{+}\right)+\frac{2\left(-4 \mathrm{i}+\xi_{1}-\xi_{2}\right)}{\xi_{1}-\xi_{2}} F_{6}\left(\xi_{2}^{+}, \xi_{1}^{-}, \xi_{1}^{+}, \xi_{2}^{-}, \xi_{3}^{-}, \xi_{3}^{+}\right) \\
& -\frac{4\left(2 i+\xi_{1}-\xi_{2}\right)}{9\left(\xi_{1}-\xi_{2}\right)} G_{6}\left(-\xi_{1}^{+},-\xi_{2}^{-},-\xi_{1}^{-},-\xi_{2}^{+},-\xi_{3}^{-},-\xi_{3}^{+}\right)+\frac{2\left(-4 i+\xi_{2}-\xi_{3}\right)}{3\left(-2 i+\xi_{2}-\xi_{3}\right)} G_{6}\left(-\xi_{1}^{-},-\xi_{1}^{+},-\xi_{2}^{+},-\xi_{3}^{+},-\xi_{2}^{-},-\xi_{3}^{-}\right) \\
& +\left(32 \mathrm{i}-52 \xi_{1}-2 \mathrm{i} \xi_{1}^{2}+36 \xi_{2}-10 \mathrm{i} \xi_{1} \xi_{2}+7 \xi_{1}^{2} \xi_{2}+12 \mathrm{i} \xi_{2}^{2}-14 \xi_{1} \xi_{2}^{2}+7 \xi_{2}^{3}+16 \xi_{3}+14 \mathrm{i} \xi_{1} \xi_{3}-7 \xi_{1}^{2} \xi_{3}\right. \\
& \left.-14 i \xi_{2} \xi_{3}+14 \xi_{1} \xi_{2} \xi_{3}-7 \xi_{2}^{2} \xi_{3}\right) \times \frac{G_{6}\left(-\xi_{1}^{-},-\xi_{1}^{+},-\xi_{2}^{-},-\xi_{2}^{+},-\xi_{3}^{-},-\xi_{3}^{+}\right)}{9\left(\xi_{1}-\xi_{2}\right)\left(2 i+\xi_{1}-\xi_{2}\right)\left(-2 i+\xi_{2}-\xi_{3}\right)} \\
& -\left(48 i \xi_{1}-24 \xi_{1}^{2}-96 i \xi_{2}+4 \xi_{1} \xi_{2}-10 i \xi_{1}^{2} \xi_{2}-4 \xi_{2}^{2}+30 i \xi_{1} \xi_{2}^{2}+\xi_{1}^{2} \xi_{2}^{2}-20 i \xi_{2}^{3}-2 \xi_{1} \xi_{2}^{3}+\xi_{2}^{4}\right. \\
& +48 i \xi_{3}+44 \xi_{1} \xi_{3}+10 i \xi_{1}^{2} \xi_{3}+4 \xi_{2} \xi_{3}-40 i \xi_{1} \xi_{2} \xi_{3}-2 \xi_{1}^{2} \xi_{2} \xi_{3}+30 i \xi_{2}^{2} \xi_{3}+4 \xi_{1} \xi_{2}^{2} \xi_{3}-2 \xi_{2}^{3} \xi_{3}-24 \xi_{3}^{2} \\
& \left.+10 \mathrm{i} \xi_{1} \xi_{3}^{2}+\xi_{1}^{2} \xi_{3}^{2}-10 \mathrm{i} \xi_{2} \xi_{3}^{2}-2 \xi_{1} \xi_{2} \xi_{3}^{2}+\xi_{2}^{2} \xi_{3}^{2}\right) \times \frac{G_{6}\left(\xi_{1}^{-}, \xi_{1}^{+}, \xi_{2}^{-}, \xi_{2}^{+}, \xi_{3}^{-}, \xi_{3}^{+}\right)}{9\left(\xi_{1}-\xi_{2}\right)\left(-2 \mathrm{i}+\xi_{1}-\xi_{2}\right)\left(\xi_{2}-\xi_{3}\right)\left(2 \mathrm{i}+\xi_{2}-\xi_{3}\right)} \\
& +\frac{2\left(4 \mathrm{i}+\xi_{1}-\xi_{2}\right)}{2 i+\xi_{1}-\xi_{2}} G_{6}\left(-\xi_{1}^{-},-\xi_{2}^{-},-\xi_{1}^{+},-\xi_{2}^{+},-\xi_{3}^{-},-\xi_{3}^{+}\right)-\frac{2\left(4 \mathrm{i}+\xi_{2}-\xi_{3}\right)\left(-6 \mathrm{i}+\xi_{2}-\xi_{3}\right)}{3\left(-2 \mathrm{i}+\xi_{2}-\xi_{3}\right)\left(2 \mathrm{i}+\xi_{2}-\xi_{3}\right)} G_{6}\left(\xi_{1}^{-}, \xi_{1}^{+}, \xi_{2}^{-}, \xi_{3}^{-}, \xi_{2}^{+}, \xi_{3}^{+}\right) \\
& -\frac{\left(2 \mathrm{i}+\xi_{2}-\xi_{3}\right)\left(4 \mathrm{i}+\xi_{2}-\xi_{3}\right)}{3\left(\xi_{2}-\xi_{3}\right)\left(-2 \mathrm{i}+\xi_{2}-\xi_{3}\right)} G_{6}\left(\xi_{1}^{-}, \xi_{1}^{+}, \xi_{3}^{-}, \xi_{3}^{+}, \xi_{2}^{-}, \xi_{2}^{+}\right)-\frac{2\left(-4 \mathrm{i}+\xi_{1}-\xi_{2}\right)\left(6 \mathrm{i}+\xi_{1}-\xi_{2}\right)}{3\left(-2 \mathrm{i}+\xi_{1}-\xi_{2}\right)\left(2 \mathrm{i}+\xi_{1}-\xi_{2}\right)} G_{6}\left(\xi_{1}^{+}, \xi_{2}^{+}, \xi_{1}^{-}, \xi_{2}^{-}, \xi_{3}^{-}, \xi_{3}^{+}\right) \\
& -\frac{\left(-2 \mathrm{i}+\xi_{1}-\xi_{2}\right)\left(-4 \mathrm{i}+\xi_{1}-\xi_{2}\right)}{3\left(\xi_{1}-\xi_{2}\right)\left(2 \mathrm{i}+\xi_{1}-\xi_{2}\right)} G_{6}\left(\xi_{2}^{-}, \xi_{2}^{+}, \xi_{1}^{-}, \xi_{1}^{+}, \xi_{3}^{-}, \xi_{3}^{+}\right)-P_{6}\left(\xi_{1}^{-}, \xi_{1}^{+}, \xi_{2}^{-}, \xi_{2}^{+}, \xi_{3}^{-}, \xi_{3}^{+}\right)
\end{aligned}
$$

$$
\begin{aligned}
& \widetilde{\rho}_{10}\left(\xi_{1}, \xi_{2}, \xi_{3}\right)=\frac{1}{27} \mathcal{N}\left(\xi_{1}, \xi_{2}, \xi_{3}\right)-\frac{4}{9} G_{5}\left(-\xi_{1}^{-},-\xi_{2}^{-},-\xi_{2}^{+},-\xi_{3}^{-},-\xi_{3}^{+}\right) \\
& +\frac{2\left(-4 i \xi_{1}+8 \mathrm{i} \xi_{2}+\xi_{1} \xi_{2}-\xi_{2}^{2}-4 \mathrm{i} \xi_{3}-\xi_{1} \xi_{3}+\xi_{2} \xi_{3}\right)}{\left(\xi_{1}-\xi_{2}\right)\left(\xi_{2}-\xi_{3}\right)} F_{6}\left(\xi_{1}^{+}, \xi_{1}^{-}, \xi_{2}^{-}, \xi_{2}^{+}, \xi_{3}^{-}, \xi_{3}^{+}\right) \\
& +\frac{2\left(4 \mathrm{i}+\xi_{2}-\xi_{3}\right)}{\xi_{2}-\xi_{3}} F_{6}\left(\xi_{1}^{+}, \xi_{1}^{-}, \xi_{2}^{-}, \xi_{3}^{-}, \xi_{3}^{+}, \xi_{2}^{+}\right)+\frac{2\left(-4 \mathrm{i}+\xi_{1}-\xi_{2}\right)}{\xi_{1}-\xi_{2}} F_{6}\left(\xi_{2}^{+}, \xi_{1}^{-}, \xi_{1}^{+}, \xi_{2}^{-}, \xi_{3}^{-}, \xi_{3}^{+}\right) \\
& -\frac{4\left(2 \mathrm{i}+\xi_{1}-\xi_{2}\right)}{9\left(\xi_{1}-\xi_{2}\right)} G_{6}\left(-\xi_{1}^{+},-\xi_{2}^{-},-\xi_{1}^{-},-\xi_{2}^{+},-\xi_{3}^{-},-\xi_{3}^{+}\right)-\frac{4\left(-4 i+\xi_{2}-\xi_{3}\right)}{3\left(-2 i+\xi_{2}-\xi_{3}\right)} G_{6}\left(-\xi_{1}^{-},-\xi_{1}^{+},-\xi_{2}^{+},-\xi_{3}^{+},-\xi_{2}^{-},-\xi_{3}^{-}\right) \\
& -\frac{2\left(-8+10 \mathrm{i} \xi_{1}-14 \mathrm{i} \xi_{2}+\xi_{1} \xi_{2}-\xi_{2}^{2}+4 \mathrm{i} \xi_{3}-\xi_{1} \xi_{3}+\xi_{2} \xi_{3}\right)}{9\left(\xi_{1}-\xi_{2}\right)\left(-2 \mathrm{i}+\xi_{2}-\xi_{3}\right)} G_{6}\left(-\xi_{1}^{-},-\xi_{1}^{+},-\xi_{2}^{-},-\xi_{2}^{+},-\xi_{3}^{-},-\xi_{3}^{+}\right) \\
& -\left(48 \mathrm{i} \xi_{1}-24 \xi_{1}^{2}-96 \mathrm{i} \xi_{2}+4 \xi_{1} \xi_{2}-10 \mathrm{i} \xi_{1}^{2} \xi_{2}-4 \xi_{2}^{2}+30 \mathrm{i} \xi_{1} \xi_{2}^{2}+\xi_{1}^{2} \xi_{2}^{2}-20 \mathrm{i} \xi_{2}^{3}-2 \xi_{1} \xi_{2}^{3}+\xi_{2}^{4}\right. \\
& +48 \mathrm{i} \xi_{3}+44 \xi_{1} \xi_{3}+10 \mathrm{i} \xi_{1}^{2} \xi_{3}+4 \xi_{2} \xi_{3}-40 \mathrm{i} \xi_{1} \xi_{2} \xi_{3}-2 \xi_{1}^{2} \xi_{2} \xi_{3}+30 i \xi_{2}^{2} \xi_{3}+4 \xi_{1} \xi_{2}^{2} \xi_{3}-2 \xi_{2}^{3} \xi_{3}-24 \xi_{3}^{2} \\
& \left.+10 \mathrm{i} \xi_{1} \xi_{3}^{2}+\xi_{1}^{2} \xi_{3}^{2}-10 \mathrm{i} \xi_{2} \xi_{3}^{2}-2 \xi_{1} \xi_{2} \xi_{3}^{2}+\xi_{2}^{2} \xi_{3}^{2}\right) \times \frac{G_{6}\left(\xi_{1}^{-}, \xi_{1}^{+}, \xi_{2}^{-}, \xi_{2}^{+}, \xi_{3}^{-}, \xi_{3}^{+}\right)}{9\left(\xi_{1}-\xi_{2}\right)\left(-2 \mathrm{i}+\xi_{1}-\xi_{2}\right)\left(\xi_{2}-\xi_{3}\right)\left(2 i+\xi_{2}-\xi_{3}\right)} \\
& -\frac{2\left(4 \mathrm{i}+\xi_{2}-\xi_{3}\right)\left(-6 \mathrm{i}+\xi_{2}-\xi_{3}\right)}{3\left(-2 \mathrm{i}+\xi_{2}-\xi_{3}\right)\left(2 \mathrm{i}+\xi_{2}-\xi_{3}\right)} G_{6}\left(\xi_{1}^{-}, \xi_{1}^{+}, \xi_{2}^{-}, \xi_{3}^{-}, \xi_{2}^{+}, \xi_{3}^{+}\right)-\frac{\left(2 i+\xi_{2}-\xi_{3}\right)\left(4 \mathrm{i}+\xi_{2}-\xi_{3}\right)}{3\left(\xi_{2}-\xi_{3}\right)\left(-2 \mathrm{i}+\xi_{2}-\xi_{3}\right)} G_{6}\left(\xi_{1}^{-}, \xi_{1}^{+}, \xi_{3}^{-}, \xi_{3}^{+}, \xi_{2}^{-}, \xi_{2}^{+}\right) \\
& -\frac{2\left(-4 \mathrm{i}+\xi_{1}-\xi_{2}\right)\left(6 \mathrm{i}+\xi_{1}-\xi_{2}\right)}{3\left(-2 \mathrm{i}+\xi_{1}-\xi_{2}\right)\left(2 \mathrm{i}+\xi_{1}-\xi_{2}\right)} G_{6}\left(\xi_{1}^{+}, \xi_{2}^{+}, \xi_{1}^{-}, \xi_{2}^{-}, \xi_{3}^{-}, \xi_{3}^{+}\right)-\frac{\left(-2 \mathrm{i}+\xi_{1}-\xi_{2}\right)\left(-4 \mathrm{i}+\xi_{1}-\xi_{2}\right)}{3\left(\xi_{1}-\xi_{2}\right)\left(2 \mathrm{i}+\xi_{1}-\xi_{2}\right)} G_{6}\left(\xi_{2}^{-}, \xi_{2}^{+}, \xi_{1}^{-}, \xi_{1}^{+}, \xi_{3}^{-}, \xi_{3}^{+}\right) \\
& -P_{6}\left(\xi_{1}^{-}, \xi_{1}^{+}, \xi_{2}^{-}, \xi_{2}^{+}, \xi_{3}^{-}, \xi_{3}^{+}\right)
\end{aligned}
$$




$$
\begin{aligned}
& \tilde{\rho}_{12}\left(\xi_{1}, \xi_{2}, \xi_{3}\right)=-\frac{1}{27} \mathcal{N}\left(\xi_{1}, \xi_{2}, \xi_{3}\right)+\frac{4}{9} G_{5}\left(-\xi_{1}^{-},-\xi_{2}^{-},-\xi_{2}^{+},-\xi_{3}^{-},-\xi_{3}^{+}\right)+2 F_{6}\left(-\xi_{1}^{-},-\xi_{1}^{+},-\xi_{2}^{-},-\xi_{2}^{+},-\xi_{3}^{+},-\xi_{3}^{-}\right) \\
& -\frac{4\left(-2 \mathrm{i} \xi_{1}+4 \mathrm{i} \xi_{2}+\xi_{1} \xi_{2}-\xi_{2}^{2}-2 \mathrm{i} \xi_{3}-\xi_{1} \xi_{3}+\xi_{2} \xi_{3}\right)}{\left(\xi_{1}-\xi_{2}\right)\left(\xi_{2}-\xi_{3}\right)} F_{6}\left(\xi_{1}^{+}, \xi_{1}^{-}, \xi_{2}^{-}, \xi_{2}^{+}, \xi_{3}^{-}, \xi_{3}^{+}\right) \\
& -\frac{2\left(4 \mathrm{i}+\xi_{2}-\xi_{3}\right)}{\xi_{2}-\xi_{3}} F_{6}\left(\xi_{1}^{+}, \xi_{1}^{-}, \xi_{2}^{-}, \xi_{3}^{-}, \xi_{3}^{+}, \xi_{2}^{+}\right)-\frac{2\left(-4 \mathrm{i}+\xi_{1}-\xi_{2}\right)}{\xi_{1}-\xi_{2}} F_{6}\left(\xi_{2}^{+}, \xi_{1}^{-}, \xi_{1}^{+}, \xi_{2}^{-}, \xi_{3}^{-}, \xi_{3}^{+}\right) \\
& +\frac{4\left(2 \mathrm{i}+\xi_{1}-\xi_{2}\right)}{9\left(\xi_{1}-\xi_{2}\right)} G_{6}\left(-\xi_{1}^{+},-\xi_{2}^{-},-\xi_{1}^{-},-\xi_{2}^{+},-\xi_{3}^{-},-\xi_{3}^{+}\right) \\
& -\left(32 \mathrm{i}-52 \xi_{1}-2 \mathrm{i} \xi_{1}^{2}+36 \xi_{2}-10 \mathrm{i} \xi_{1} \xi_{2}+7 \xi_{1}^{2} \xi_{2}+12 \mathrm{i} \xi_{2}^{2}-14 \xi_{1} \xi_{2}^{2}+7 \xi_{2}^{3}+16 \xi_{3}+14 \mathrm{i} \xi_{1} \xi_{3}-7 \xi_{1}^{2} \xi_{3}\right. \\
& \left.-14 i \xi_{2} \xi_{3}+14 \xi_{1} \xi_{2} \xi_{3}-7 \xi_{2}^{2} \xi_{3}\right) \times \frac{G_{6}\left(-\xi_{1}^{-},-\xi_{1}^{+},-\xi_{2}^{-},-\xi_{2}^{+},-\xi_{3}^{-},-\xi_{3}^{+}\right)}{9\left(\xi_{1}-\xi_{2}\right)\left(2 \mathrm{i}+\xi_{1}-\xi_{2}\right)\left(-2 \mathrm{i}+\xi_{2}-\xi_{3}\right)} \\
& -\frac{2\left(4 i+\xi_{1}-\xi_{2}\right)}{2 i+\xi_{1}-\xi_{2}} G_{6}\left(-\xi_{1}^{-},-\xi_{2}^{-},-\xi_{1}^{+},-\xi_{2}^{+},-\xi_{3}^{-},-\xi_{3}^{+}\right) \\
& +2\left(24 i \xi_{1}-12 \xi_{1}^{2}-48 i \xi_{2}-16 \xi_{1} \xi_{2}-14 i \xi_{1}^{2} \xi_{2}+16 \xi_{2}^{2}+24 i \xi_{1} \xi_{2}^{2}+5 \xi_{1}^{2} \xi_{2}^{2}-10 i \xi_{2}^{3}-10 \xi_{1} \xi_{2}^{3}+5 \xi_{2}^{4}\right. \\
& +24 i \xi_{3}+40 \xi_{1} \xi_{3}+14 i \xi_{1}^{2} \xi_{3}-16 \xi_{2} \xi_{3}-20 i \xi_{1} \xi_{2} \xi_{3}-10 \xi_{1}^{2} \xi_{2} \xi_{3}+6 i \xi_{2}^{2} \xi_{3}+20 \xi_{1} \xi_{2}^{2} \xi_{3}-10 \xi_{2}^{3} \xi_{3}-12 \xi_{3}^{2} \\
& \left.-4 \mathrm{i} \xi_{1} \xi_{3}^{2}+5 \xi_{1}^{2} \xi_{3}^{2}+4 \mathrm{i} \xi_{2} \xi_{3}^{2}-10 \xi_{1} \xi_{2} \xi_{3}^{2}+5 \xi_{2}^{2} \xi_{3}^{2}\right) \times \frac{G_{6}\left(\xi_{1}^{-}, \xi_{1}^{+}, \xi_{2}^{-}, \xi_{2}^{+}, \xi_{3}^{-}, \xi_{3}^{+}\right)}{9\left(\xi_{1}-\xi_{2}\right)\left(-2 \mathrm{i}+\xi_{1}-\xi_{2}\right)\left(\xi_{2}-\xi_{3}\right)\left(2 \mathrm{i}+\xi_{2}-\xi_{3}\right)} \\
& -\frac{2\left(-4 i+\xi_{2}-\xi_{3}\right) G_{6}\left(-\xi_{1}^{-},-\xi_{1}^{+},-\xi_{2}^{+},-\xi_{3}^{+},-\xi_{2}^{-},-\xi_{3}^{-}\right)}{3\left(-2 \mathrm{i}+\xi_{2}-\xi_{3}\right)}+\frac{8\left(-3 \mathrm{i}+\xi_{2}-\xi_{3}\right)\left(4 \mathrm{i}+\xi_{2}-\xi_{3}\right)}{3\left(-2 \mathrm{i}+\xi_{2}-\xi_{3}\right)\left(2 \mathrm{i}+\xi_{2}-\xi_{3}\right)} G_{6}\left(\xi_{1}^{-}, \xi_{1}^{+}, \xi_{2}^{-}, \xi_{3}^{-}, \xi_{2}^{+}, \xi_{3}^{+}\right) \\
& +\frac{\left(2 i+\xi_{2}-\xi_{3}\right)\left(4 i+\xi_{2}-\xi_{3}\right)}{3\left(\xi_{2}-\xi_{3}\right)\left(-2 i+\xi_{2}-\xi_{3}\right)} G_{6}\left(\xi_{1}^{-}, \xi_{1}^{+}, \xi_{3}^{-}, \xi_{3}^{+}, \xi_{2}^{-}, \xi_{2}^{+}\right)+\frac{2\left(-4 i+\xi_{1}-\xi_{2}\right)\left(6 i+\xi_{1}-\xi_{2}\right)}{3\left(-2 i+\xi_{1}-\xi_{2}\right)\left(2 i+\xi_{1}-\xi_{2}\right)} G_{6}\left(\xi_{1}^{+}, \xi_{2}^{+}, \xi_{1}^{-}, \xi_{2}^{-}, \xi_{3}^{-}, \xi_{3}^{+}\right) \\
& +\frac{\left(-2 \mathrm{i}+\xi_{1}-\xi_{2}\right)\left(-4 \mathrm{i}+\xi_{1}-\xi_{2}\right)}{3\left(\xi_{1}-\xi_{2}\right)\left(2 \mathrm{i}+\xi_{1}-\xi_{2}\right)} G_{6}\left(\xi_{2}^{-}, \xi_{2}^{+}, \xi_{1}^{-}, \xi_{1}^{+}, \xi_{3}^{-}, \xi_{3}^{+}\right)+P_{6}\left(\xi_{1}^{-}, \xi_{1}^{+}, \xi_{2}^{-}, \xi_{2}^{+}, \xi_{3}^{-}, \xi_{3}^{+}\right)
\end{aligned}
$$

The rest is easily reproduced by applying the symmetry relations (110). The explicit forms of $F_{a}, G_{a}$ and $P_{a}$ in terms of $\omega\left(\xi_{i}, \xi_{j}\right)$ are too lengthly to be reproduced here.

\section{Appendix : References}

[1] Britta Aufgebauer and Andreas Klümper: Finite temperature correlation functions from discrete functional equations, J. Phys. A: Math. Theor. 45 (2012) 345203.

[2] H. M. Babujian, Exact solution of the one-dimensional isotropic Heisenberg chain witgh arbitrary spins S, Phys. Lett. A 90 (1982) 479.

[3] H. Boos and F. Göhmann, On the physical part of the factorized correlation functions of the XXZ chain, J. Phys. A 42 (2009) 315001.

[4] H. Boos, F. Göhmann, A. Klümper and J. Suzuki, Factorization of multiple integrals representing the density matrix of a finite segment of the Heisenberg spin chain, J. Stat. Mech. (2006) P04001.

[5] -, Factorization of the finite temperature correlation functions of the XXZ chain in a magnetic field, J. Phys. A 40 (2007) 10699.

[6] H. Boos, M. Jimbo, T. Miwa, F. Smirnov and Y. Takeyama, Hidden Grassmann structure in the XXZ model, Comm. Math. Phys. 272 (2007) 263. 
[7] —, Hidden Grassmann structure in the XXZ model II: creation operators, Comm. Math. Phys. 286 (2009) 875.

[8] H. E. Boos and V. E. Korepin, Quantum spin chains and Riemann zeta function with odd arguments, J. Phys. A 34 (2001) 5311.

[9] A. H. Bougourzi and R. A. Weston, N-point correlation functions of the spin-1 XXZ model, Nucl. Phys. B 417 (1994) 439.

[10] J. Damerau, F. Göhmann, N. P. Hasenclever and A. Klümper, Density matrices for finite segments of Heisenberg chains of arbitrary length, J. Phys. A 40 (2007) 4439.

[11] T. Deguchi and C. Matsui, Correlation functions of the integrable higher-spin $X X X$ and XXZ spin chains through the fusion method, Nucl. Phys. B 831 (2010) 359.

[12] F. H. L. Essler, H. Frahm, F. Göhmann, A. Klümper and V. E. Korepin, The One-Dimensional Hubbard Model (Cambridge University Press, 2005).

[13] F. Göhmann, N. P. Hasenclever and A. Seel, The finite temperature density matrix and two-point correlations in the antiferromagnetic XXZ chain, J. Stat. Mech. (2005) P10015.

[14] F. Göhmann, A. Klümper and A. Seel, Integral representations for correlation functions of the XXZ chain at finite temperature, J. Phys. A 37 (2004) 7625.

[15] —, Integral representation of the density matrix of the $X X Z$ chain at finite temperature, J. Phys. A 38 (2005) 1833.

[16] F. Göhmann, A. Seel and J. Suzuki, Correlation functions of the integrable isotropic spin-1 chain at finite temperature, J. Stat. Mech. (2010) P11011

[17] F. Göhmann and J. Suzuki, Quantum spin chains at finite temperature, in "New Trends in Quantum Integrable Systems" World Scientific, Singapore (2010) 81100.

[18] M. Idzumi, Level-2 irreducible representations of $U(q)(s l(2))$, vertex operators, and their correlations, Int. J. Mod. Phys. A 9 (1994) 4449.

[19] M. Jimbo, K. Miki, T. Miwa and A. Nakayashiki, Correlation functions of the XXZ model for $\Delta<-1$, Phys. Lett. A 168 (1992) 256.

[20] M. Jimbo and T. Miwa, Quantum KZ equation with $|q|=1$ and correlation functions of the XXZ model in the gapless regime, J. Phys. A 29 (1996) 2923.

[21] M. Jimbo, T. Miwa and F. Smirnov, Hidden Grassmann structure in the XXZ model III: introducing Matsubara direction, J. Phys. A 42 (2009) 304018.

[22] —, On one-point functions of descendants in Sine-Gordon model, in "New Trends in Quantum Integrable Systems” World Scientific, Singapore (2010) 117-138. 
[23] G. Jüttner, A. Klümper and J. Suzuki, From fusion hierarchy to excited state TBA, Nucl. Phys. B 512 (1998) 581

[24] N. Kitanine, Correlation functions of the higher spin XXX chains, J. Phys. A 34 (2001) 8151.

[25] N. Kitanine, K. Kozlowski, J. M. Maillet, N. A. Slavnov and V. Terras, On correlation functions of integrable models associated with the six-vertex R-matrix, J. Stat. Mech. (2007) P01022.

[26] —, Algebraic Bethe ansatz approach to the asymptotic behavior of correlation functions, J. Stat. Mech. (2009) P04003.

[27] N. Kitanine, J. M. Maillet and V. Terras, Correlation functions of the XXZ Heisenberg spin- $\frac{1}{2}$ chain in a magnetic field, Nucl. Phys. B 567 (2000) 554.

[28] A. Klümper and P. A. Pearce, Conformal weights of RSOS lattice models and their fusion hierarchy, Physica A 183, (1992) 304-350

[29] A. Klümper, Thermodynamics of the anisotropic spin-1/2 Heisenberg chain and related quantum chains, Z. Phys. B 91 (1993) 507.

[30] P. P. Kulish, N. Yu. Reshetikhin and E. K. Sklyanin, Yang-Baxter equation and representation theory: I, Lett. Math. Phys. 5 (1981) 393.

[31] P. P. Kulish and E. K. Sklyanin, Quantum spectral transform method - recent developments, in Lecture Notes in Physics 151, 61-119 (Springer Verlag, Berlin, 1982).

[32] A. Seel, T. Bhattacharyya, F. Göhmann and A. Klümper, A note on the spin-1/2 XXZ chain concerning its relation to the Bose gas, J. Stat. Mech. (2007) P08030.

[33] A. Seel, F. Göhmann and A. Klümper, From multiple integrals to Fredholm determinants, Prog. Theor. Phys. Suppl. 176 (2008) 375.

[34] J. Suzuki, Spinons in magnetic chains of arbitrary spins at finite temperatures, J. Phys. A 32 (1999) 2341.

[35] M. Suzuki, Transfer-matrix method and Monte Carlo simulation in quantum spin systems, Phys. Rev. B 31 (1985) 2957.

[36] J. Suzuki, Y. Akutsu and M. Wadati, A New approach to Quantum Spin Chains at Finite Temperature, J. Phys. Soc. Jpn 59 (1990) 2667-2680.

[37] M. Suzuki and M. Inoue, The ST-transformation approach to analytic solutions of quantum systems. I. General formulations and basic limit theorems, Prog. Theor. Phys. 78 (1987) 787.

[38] L. A. Takhtajan, The picture of low-lying excitations in the isotropic Heisenberg chain of arbitrary spins, Phys. Lett. A 87 (1982) 479. 
[39] A. B. Zamolodchikov and A. V. Fateev, A model factorized S-matrix and an integrable spin-1 Heisenberg chain, Yad. Fiz. 32 (1980) 581. 